$12-1-2020$

\title{
Shughuli za UNHCR na Washirikiza Ulinzi kwa Jamii baina ya Sekta katika Maeneo ya Masharik na, Pembe ya Afrika na Maziwa Makuu
}

Charles Mballa

Josephine Ngebeh

Machtelt De Vriese

Katie Drew

Abigayil Parr

See next page for additional authors

Follow this and additional works at: https://knowledgecommons.popcouncil.org/departments_sbsr-rh How does access to this work benefit you? Let us know!

\section{Recommended Citation}

Mballa, Charles, Josephine Ngebeh, Machtelt De Vriese, Katie Drew, Abigayil Parr, and Chi-Chi Undie. 2020. "Shughuli za UNHCR na Washirikiza Ulinzi kwa Jamii baina ya Sekta katika Maeneo ya Masharik na, Pembe ya Afrika na Maziwa Makuu." Nairobi: UNHCR and Population Council. 


\section{Authors}

Charles Mballa, Josephine Ngebeh, Machtelt De Vriese, Katie Drew, Abigayil Parr, and Chi-Chi Undie 


\section{SHUGHULI ZA TUME YA WAKIMBIZI YA}

UMOJA WA MATAIFA (UNHCR) NA WASHIRIKI ZA ULINZI KWA JAMII BAINA YA SEKTA KATIKA MAENEO YA MASHARIKI NA PEMBE YA AFRIKA NA MAENEO YA MAZIWA MAKUU

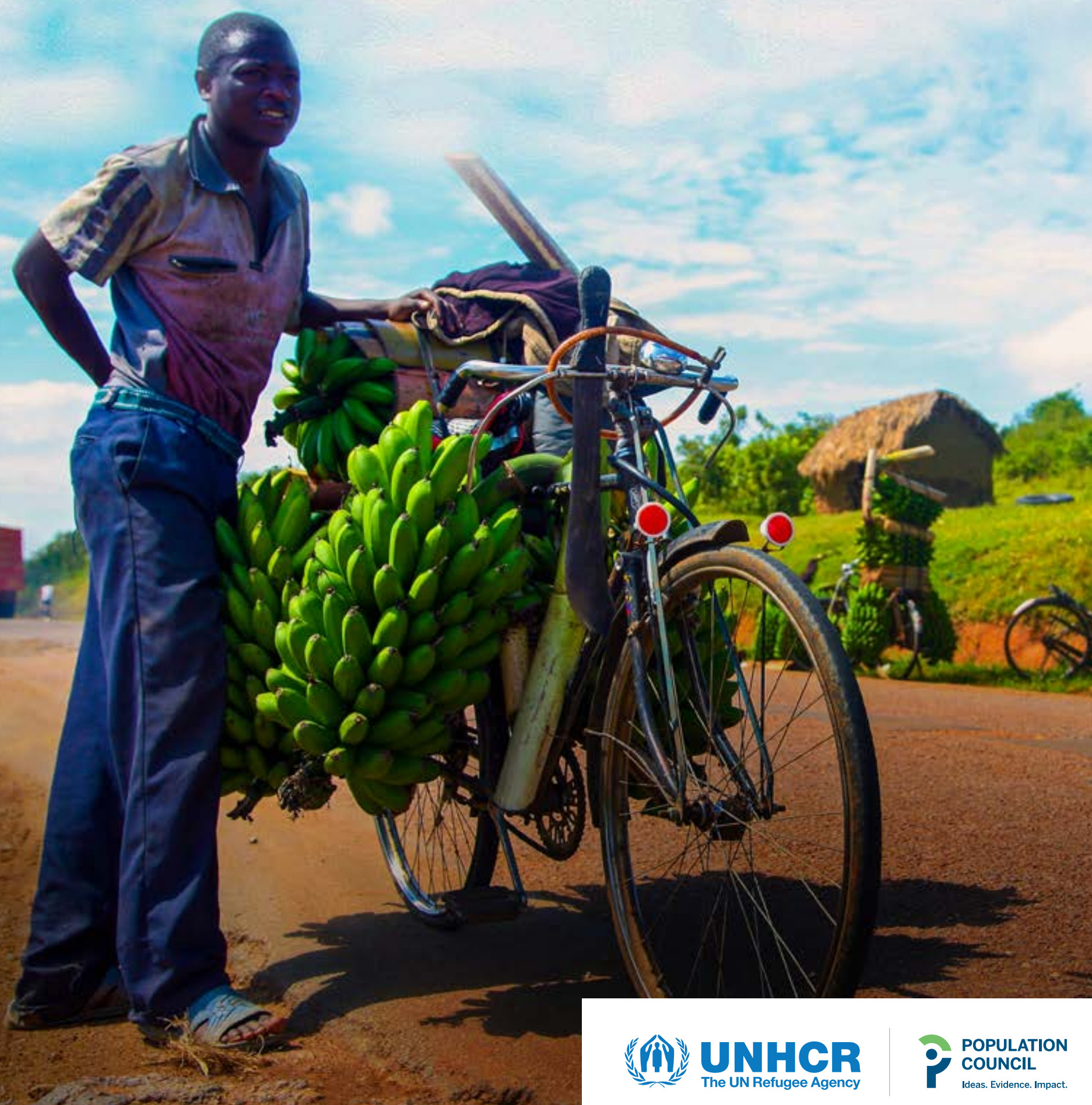




\section{Nukuu inayopendekezwa}

Charles Mballa, Josephine Ngebeh, Machtelt De Vriese, Katie Drew, Abigayil Parr, Chi-Chi Undie. 2020. Shughuli za UNHCR na Washirikiza Ulinzi kwa Jamii baina ya Sekta katika Maeneo ya Masharik na, Pembe ya Afrika na Maziwa Makuu. UNHCR na Baraza ya Idadi ya Watu 


\section{YALIYOMO}

VIFUPISHO

DIBAJI

UTANGULIZI

4

METHODOLOJIA

MUHTASARI WA DESTURI

HARAKATI ZINAZOHUSIANA NA UGONJWA WA COVID

1. KUWAKINGA WATOTO NA VIJANA

9

2. ULINZI WA KIJAMII

3. DHULMA ZA KIMAPENZI NA KIJINSIA/

KUPUNGUZA HATARI/ KUITIKIA

4. DHULMA ZA KIMAPENZI NA KIJINSIA/

KUPUNGUZA HATARI/ KUITIKIA 


\section{VIFUPISHO}

\begin{tabular}{|c|c|}
\hline AGD & Umri, Jinsia na Uanuwai \\
\hline ARRA & Shirika la Maslahi ya Wakimbizi na Wanaorudi \\
\hline CBAC & Kamati ya Kijamii ya Vijana Wanaobalehe \\
\hline CBP & Ulinzi wa Kijamii \\
\hline CCCT & Timu ya Muungano wa Utunzi kwa Jamii \\
\hline CHH & Nyumba Inayoongozwa na Mtoto \\
\hline CHW & Mhudumu wa Afya ya Jamii \\
\hline CORPS & Wataalam Wanaomilikiwa na Jamii \\
\hline CPC & Kamati ya Ulinzi kwa Watoto \\
\hline CPV & Watu Wanaojitolea kwa Ulinzi wa Watoto \\
\hline CRRF & Mfumo wa Kina wa Mwitikio kwa Wakimbizi \\
\hline CWC & Kamati ya Maslahi ya Watoto \\
\hline DICAC & Tume ya Maendeleo na Msaada Baina ya Makanisa \\
\hline DRC & Baraza ya Wakimbizi nchini Denmark \\
\hline DHR & Shirika la Direction de l'Hydraulique Rurale \\
\hline EHAGL & Mashariki na Pembe ya Afrika na Maziwa Makuu \\
\hline FDP & Maeneo ya Ugavi wa Chakula \\
\hline GE & Usawa wa Jinsia \\
\hline HCW & Wahudumu wa Afya \\
\hline HIAS & Jumuiya ya Misaada ya Wahamiaji wa Kiebrania \\
\hline IDP & Wakimbizi wa Ndani \\
\hline IPV & Dhulma kati ya Wapenzi \\
\hline IRC & Shirika la International Rescue Committee \\
\hline KAP & Ufahamu, Mitazamo na Mazoea \\
\hline LWF & Shirika la Lutheran World Federation \\
\hline MEARL & Kufuatilia, Kutathmini, Kuwajibisha, Utafiti, na Mafunzo \\
\hline MHPSS & Msaada wa Afya ya Kiakili na Kijamii \\
\hline MINEMA & Wizara Inayoshughulikia Udhibiti wa Dharura \\
\hline MoA & Mkataba wa Makubaliano \\
\hline MWC & Kamati ya Maji Sokoni \\
\hline NGO & Mashirika Yasiyo ya Serikali \\
\hline NRC & Baraza ya Wakimbizi ya Norway \\
\hline OPM & Ofisi ya Waziri Mkuu \\
\hline PPA & Makubaliano ya Ushirikiano wa Mradi \\
\hline PRC & Utunzi wa Baada ya Kubakwa \\
\hline PTA & Vyama vya Wazazi na Walimu \\
\hline RADO & Shirika la Ukarabati na Maendeleo \\
\hline RCC & Kamati Kuu ya Wakimbizi \\
\hline RCCE & Mawasiliano kuhusu Hatari na Kushirikisha Jamii \\
\hline RCW & Wafanyikazi wa Jamii ya Wakimbizi \\
\hline REF & Majadiliano Yanayohusisha Wakimbizi \\
\hline ROV & Wakimbizi wa Kujitolea kuwasilisha Huduma \\
\hline SCl & Shirika la Save the Children International \\
\hline SF & Baraza ya Washikadau \\
\hline SG & Kikundi cha Uendeshaji \\
\hline SGBV & Dhulma ya Kimapenzi na Kijinsia \\
\hline TB & Kifua Kikuu \\
\hline TdH & Shirika la Terre des Hommes \\
\hline TWG & Kikundi cha Utendakazi cha Kiufundi \\
\hline UN & Umoja wa Mataifa \\
\hline UNFPA & Hazina ya Umoja wa Mataifa kuhusu Idadi ya Watu \\
\hline UNHCR & Tume ya Wakimbizi ya Umoja wa Mataifa \\
\hline UNICEF & Hazina ya Dharura ya Watoto ya Umoja wa Mataifa \\
\hline VSLA & Chama cha Kuweka Akiba kwa Hiari na Kutoa Mikopo \\
\hline WASH & Maji, Usafishaji na Usafi \\
\hline WEO & Ofisi ya Elimu ya Woreda \\
\hline WFP & Shirika la Chakula Ulimwenguni \\
\hline ZTVA & Muungano wa Kijiji Kukatiza Kuvumilia Dhulma \\
\hline
\end{tabular}




\section{DIBAJI}

M

uhtasari huu ulitengenezwa kwa ushirikiano wa Ofisi ya Maeneo ya UNHCR ya Mashariki na Pembe ya Afrika na Maziwa Makuu, pamoja na Baraza ya Idadi ya Watu ncini Kenya. Muhtasari huu unaleta pamoja mikakati mbalimbali ya ubunifu ya ulinzi wa jamii. Muhtasari huu umearifiwa na desturi ya ulinzi wa jamii iliyoombwa kutoka kwa washiriki, wakimbizi na wenyeji pamoja na washikadau wengine. Maeneo ya Mashariki na Pembe ya Afrika na Maziwa Makuu yana wakimbizi milioni 4. Idadi hii ya watu wakimbizi inabainishwa na uanuwai wao na inajumuisha zaidi ya asilimia 80 ya wanawake, wasichana, wavulana na vijana waliobalehe walio na mahitaji mengi ya ulinzi.

Kwa kufuatia mtazamo wa jamii yote ambayo inaunga mkono Ushikamano wa Ulimwengu kwa Wakimbizi, muhtasari huu ni matokeo ya ushirikiano wa muda mrefu kati ya Ofisi ya Maeneo ya UNHCR na Baraza ya Idadi ya Watu nchini Kenya ulioanza katika mwaka wa 2014. Ofisi ya Maeneo na Baraza ya Idadi ya Watu nchini Kenya zilishirikiana, kwa kuongozwa na mfumo wa kiufundi wa ushirikiano unaolenga kuimarisha miradi na kugawana habari katika mazingira ya wakimbizi.

Muhtasari huu ndio ushahidi wa azimio linaloonyeshwa na taasisi zote mbili za kufanyisha kazi mwongozo wa kimsingi wa ulinzi, ikijumuisha: Sera na Mitazamo ya Umri, Jinsia na Uanuwai (2018); Miongozo ya Uwajibikaji kwa Idadi ya Watu Inayoathirika; Miongozo ya Kamati Maalum Baina ya Mashirika, Kujumuishwa kwa Watu wanaoishi na Ulemavu katika Hatua za Kibinadamu na mifumo ingine ya ulinzi ambayo inaimarisha malengo ya Ushikamano wa Ulimwengu kwa Wakimbizi unaothibitishwa na Mataifa Wanachama wa Baraza Kuu la Umoja wa Mataifa. Muhtasari huu pia unapenda kuonyesha mitazamo ya washikadau mbalimbali katika kufikia matokeo ya kijumla ya ulinzi, na kuorodhesha jinsi ya kujikinga kwa kuzingatia umri, jinsia na uanuwai na muitikio kwa COVID- 19 kwa njia ya kuimarika.

Ni matumaini yetu kuwa muhtasari huu utatumika kama zana mwafaka na washikadau ili kuboresha utoaji ulinzi; kukuza ushiriki wa moja kwa moja wa Watu Husika na wenyeji katika kufanya maamuzi; na kunakili desturi bunifu kwa njia ambayo itaathiri maisha na mahitaji mbalimbali ya ulinzi ya mamilioni ya wakimbizi katika eneo.

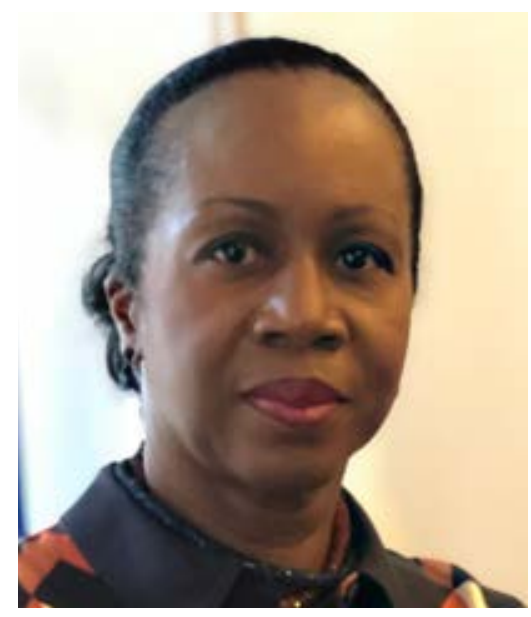

Clementine Awu Nkweta Salami Mkurugenzi Mkuu wa UNHCR Ofisi ya Eneo La Mashariki na Pembe ya Afrika na Maziwa Maku

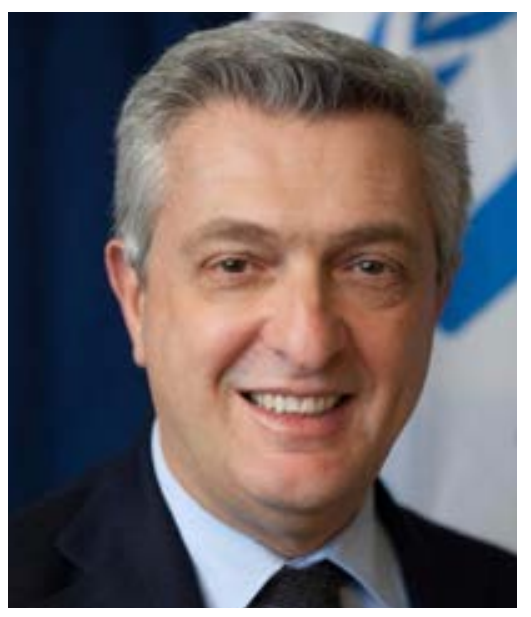

Filippo Grandi

Kamishina Mkuu wa Wakimbizi wa Umoja wa Mataifa

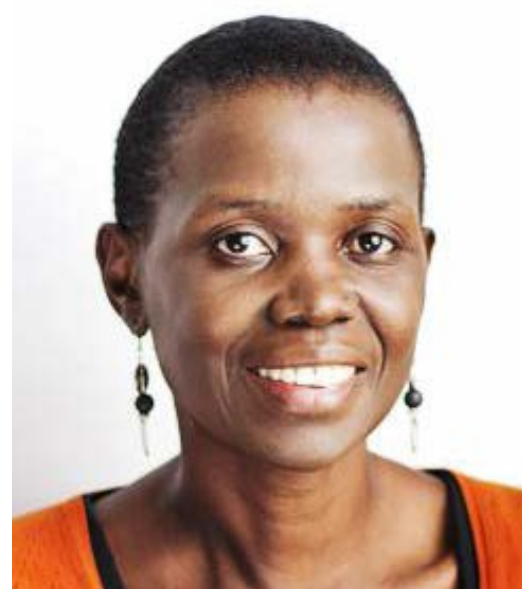

Harriet Birungi

Mkurugenzi Nchini \& Mshirika Baraza ya Idadi ya Watu, Kenya 


\section{METHODOLOJIA}

$\mathrm{O}$ fisi ya UNHCR katika Eneo la EHAGL husimamia jumla ya nchi 11, nazo ni: Burundi, Djibouti, Eritrea, Ethiopia, Kenya, Rwanda, Somalia, South Sudan, Sudan, Tanzania, na Uganda. Kitengo cha UNHCR cha Ulinzi wa Kijamii (CBP) kilialika watendaji wa nchi zote kutambua desturi muhimu za kabla na katika kipindi cha COVID- 19. Ili kuwezesha mchakato wa utambuzi, mizani ya ukadiriaji kutoka kwa UNHCR² uliwasilishwa kwa Watu Maalum wa Sekta za UNHCR ambao waliulizwa kueneza mizani hiyo na hati ya methodolojia ya UNHCR ili kulinganisha ${ }^{3}$ na utendakazi wao. Timu kutoka kwa sekta mbalimbali zilipendekezwa kama mbinu ya kuhakikisha kuwa utambuzi wa harakati utakuwa jumuishi kama inavyowezekana na kuwa Watu Husika walihusishwa pamoja na washiriki wanaotekeleza huduma. Kiolezo cha kuwasilisha harakati cha UNHCR ${ }^{4}$ pia kilipendekezwa kama muundo wa utendakazi utakaotumika katika kuandika na kuwasilisha desturi zao.

Ili kuhimiza mawasilisho, mapendekezo haya hayakuwasilishwa kama maagizo.

Hati ya methodolojia ya UNHCR ya kukusanya desturi inaanisha makundi matatu ya kuainisha desturi kwenye eneo la tukio, nazo ni, desturi zinazotia moyo, desturi zinazojitokeza na mifano ya visa. Makundi haya yamefafanuliwa vifuatavyo.

\begin{tabular}{l|l} 
Kikundi & Ufafanuzi \\
\hline Desturi Inayotia moyo & $\begin{array}{l}\text { Desturi ambayo imehakikiwa kufanya kazi vizuri na kuwa na matokeo endelevu na inahusu } \\
\text { ulinzi na/aoina uwezo wa kubadilisha watu binafsi na/au jamii kama inavyoonyeshwa na } \\
\text { matokeo ya kuaminika. Inaweza kutumika kama muundo utakaojirudia na kukuzwa zaidi. }\end{array}$ \\
\hline Desturi Inayojitokeza & $\begin{array}{l}\text { Desturi inayoonyesha ashirio za mapema za kuwa na matokeo chanya ya kubadilisha maisha } \\
\text { ya watu binafsi na/au jamii. }\end{array}$ \\
\hline Mfano wa Kisa & $\begin{array}{l}\text { Desturiau sehemu ya desturiambayo ilizingatia au ilijaribu kuzingatia (na kupata changamoto), } \\
\text { kigezo kilichowekwa kisichojitaji ushahidi au maamuzi yoyote kuhusiana na matokeo au } \\
\text { uendelevu wake. Inaweza kutoa ufahamu na mafunzo katika kupanga miradi katika siku za } \\
\text { usoni hata ikiwa haiambatani na vigezo fulani. }\end{array}$
\end{tabular}

Kama ilivyoorodheshwa katika hati ya methodolojia ya UNHCR,

'Desturi za kutia moyo ni zile zinazotimiza viwango vya juu zaidi na kuonyesha ushahidi na zitakaguliwa zaidi. Desturi zinazojitokeza ni zile zinazoonyesha dalili au zenye uwezekano wa kuwa na matokeo. ... Mfano wa visa ni desturi ambazo zimetimiza mahitaji ya kimsingi na zinaonyesha dalili au zina uwezekano wa kuwa na matokeo. Desturi hizi huenda zikawa katika awamu tofauti katika msururu wa mradi na kuwa na kiasi au ubora wa ushahidi katika matokeo. [desturi zinazojitokeza na mifano ya visa] zina umuhimu wa kuratibiwa kwani zinaweza kutumika katika upangaji wa miradi katika siku za usoni.5

Mikutano miwili ilifanyika kupitia mtandao na Watu Maalum wa Sekta ya UNHCR ili kufafanua kusudi la muhtasari huu, kushinikiza umuhimu wake na kuongeza mawasilisho ya utendakazi wa nchi.

2 UNHCR (2019). Methodology and Background Document: Collecting Practices and Case Studies - Child Protection, Community-Based Protection, Gender Equality, Sexual and Gender-Base Violence, pp. 12-18.

3 Ibid.

4 Ibid., pp. 16-17.

5 UNHCR (2019). Methodology and Background Document: Collecting Practices and Case Studies - Child Protection, Community-Based Protection, Gender Equality, Sexual and Gender-Base Violence, p. 7. 
Katika mwezi wa Septemba 2020, timu ya watu watatu ya ukaguzi ilitathmini kila wasilisho kwa kujitegemea, ikaainisha kila moja kulingana na uainisho kutumia maelezo ya 'desturi ya kutia moyo,'desturi inayojitokeza,' na 'mfano wa kisa.' Kwa visa ambavyo utathmini wa wakaguzi haukuwa sawa, utathmini uliotolewa na idadi kubwa ya wakaguzi (2 kati ya 3) ulitumika kama uainisho wa mwisho. Pale ambapo kulizuka kuhitilafiana (kutokana na ushiriki/uhusiano wa mkaguzi na desturi inayotathminiwa) mkaguzi anayehusika alitengwa kushiriki utathmini uliokuwa ukizungumziwa. Isitoshe, warsha tatu za maeneo zilifanyika kupitia mtandao katika mwezi wa September 2020, zikiwaleta pamoja wafanyakazi wa nchi ili kujadiliana mawasilisho yao, kutoa maonyesho yao kuhusiana na desturi waliyochagua, kushinikiza ufahamu wa wawasilishaji kuhusu uainisho wa desturi hizi na kukubaliana kuhusu uainisho wa mwisho uliotolewa na watathmini. Warsha ya kwanza ilikusudiwa kuangazia desturi yoyote iliyowasilishwa kutoka nchi za Eritrea, Djibouti, Somalia, Sudan Kusini na Sudan; huku warsha ya pili ikiangazia Ethiopia, Kenya na Uganda; na ya tatu ikileta pamoja Burundi, Rwanda na Tanzania. Baada ya warsha hizi, wawasilishaji walipewa siku kadhaa kuwasilisha habari zaidi kuhusu desturi yoyote iliyokuwa imewasilishwa, ikiwa kulikuwa na ushahidi wowote ambao haukujumuishwa katia kiolezo ya uwasilishaji ambao ungesaidia katika uainishwaji wa desturi hii. Warsha hizi tatu zilizofanyika kupitia mtandao ziliwaleta pamoja jumla ya washiriki 120.

Mawasilisho yote yalizingatiwa hata ikiwa kiolezo cha uwasilishi kilitumika katika kuwasilisha desturi au la (mawasilisho ambayo hayakuzingatiwa nia yale ambayo yalijaribu kueleza desturi kutumia sentensi 1 au 2 na hivyo kukosa kutoa habari ya kutosha). Huku hili likiwa ni changamoto katika kuwasilisha desturi kwa njia ya usawa, jambo hili liliongeza ushiriki na kuakikisha kujumuishwa kwa desturi bunifu kutoka kwa maeneo yote: Muhtasari huu una takriban desturi 70 kutoka kwa nchi 10 za utendakazi.

Muhtasari huu umepangwa kwa sehemu mbili kuu: Desturi zinazohusiana na COVID- 19 na desturi zisizohusiana na COVID- 19. Sehemu hizi kuu zimepangwa zaidi katika mada kuu za UNHCR ambazo ni: Ulinzi wa Watoto na Vijana, Ulinzi wa Kijamii, Usawa wa Kijinsia, Dhulma za Kimapenzi na Kijinsia (SGBV)/Kupunguza Hatari/Kuitikia. Ni vyema kutambua kuwa Kuwajibika kwa Waathiriwa kunahusisha maeneo yote yanayoangaziwa kama inavyoakisiwa katika muhtasari huu.

Kila desturi inaelezwa na orodha ya matokeo pia ikijumuishwa kwa Desturi za Kutia moyo, na mara nyingi kwa Desturi Zinazojitokeza. Kwa ajili ya ufupisho, maelezo ya desturi hayaingilii katika masuala kama vile changamoto za utekelezaji ila unapaswa kujaribu kuwasilisha habari ya kutosha ili msomaji aelewe malengo na vipengele vya kila mkakati. Nambari za mawasiliano pia zinatolewa ili msomaji aweze kufuatilia moja kwa moja na waliobuni desturi na/au wanaotekeleza desturi hizi kwa mara ya kwanza. Kwa hakika, inatarajiwa kuwa muingiliano kama huu utafanyika ndani na kati ya nchi husika na kuwa muhtasari huu utazidi kuhimiza kutafuta na kugawana habari.

Muingiliano kati ya maeneo husika yameangaziwa na husababisha kuainishwa kwa desturi usiokuwa na dosari kwote. Hata hivyo, ukweli huu unaashiria muingiliano uliopo katika maeneo husika, jambo ambalo linaonekana kuwa sawa.

6 Two Innovation Officers, with the UNHCR EHAGL Bureau, and with the Innovation Service (Global), respectively; and a Population Council Senior Associate. All reviewers were based in Nairobi, Kenya. 


\section{MAMBO MAKUU KUHUSU DESTURI ZA KUTIA MOYO}

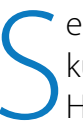
ehemu hii ya muhtasari huu inaishia na mambo makuu kutoka kwa desturi za kutia moyo zilizotambuliwa katika eneo kulingana na mawasilisho ya hiari kutoka kwa washikadau. Inalenga matokeo au athari zinazotokana na desturi hizi. Habari zaidi kuhusu kila desturi iliyowasilishwa ili kujumuishwa kwenye muhtasari (ikijumuisha desturi zote zinazotia moyo) zimeorodheshwa katika sehemu iliyosalia ya hati hii.

\section{Desturi ya Kutia moyo kutoka Ethiopia: Kukadiria na Kupanga wa Elimu na Mtazamo wa Kuhusisha Jamii}

\section{Matokeo}

- Tangu kuanzishwa kwa mtazamo huu katika mwaka wa 2015, kumekuwa na uhudhurio na usaliaji bora katika kiwango cha shule.

- Kulikuwa na asilimia 83 ya hudhurio katika kiwango cha shule ya msingi katika mwaka wa 2019 na ongezeko la asilimia 53 ya usajili katika kiwango cha sule ya sekondari katika shule za wakimbizi na zile za jamii inayoishi katika eneo kwa jumla katika nusu ya kwanza ya mwaka wa 2020.

\section{Desturi ya Kutia moyo kutoka nchi ya Rwanda: Kikundi cha Kufanya kazi ya Usafi katika Jamii na Jitihada za Pamoja za Jamii za Kukinga COVID-19, Ikiwajumuisha Wanamuziki wa Rwanda}

\section{Matokeo}

- Ikiwa kama hatua ya pamoja ya kuzuia COVID- 19, jamii ya wakimbizi imejenga sehemu 122 zaidi za uoshaji mikono ili kukuza usafi kambini.

- Matokeo kutoka kwa utafiti kuhusu Ufahamu, Mitazamo na Mazoea (KAP) uliashiria kuwa ufahamu kuhusu COVID- 19 kambini ulikuwa juu na kuwa wakimbizi wengi katika kambi husika walitambua mawasiliano ya afya ya kijamii kuwa asili kuu ya habari. Isitoshe, wakimbizi katika walipata alama ya juu katika KAP ya uoshaji mikono (ikilinganishwa na mazoea mengine ya usafi) hivyo kuakisi kwa kiasi athari chanya ya jitihada za pamoja za jamii katika kuanzisha maeneo ya kuosha mikono.

- Wanawake walipata alama a chini kuliko wanaume kuhusu ufahamu unaousiana na COVID- 19 kutokana na wao kutojua kusoma na kuandika na kutoweza kufikilia simu/redio.

\section{Desturi ya Kutia moyo kutoka nchi ya Rwanda: Vyama vya Kuweka Akiba kwa Hiari na Kutoa Mikopo (VSLAs)}

\section{Matokeo}

Kambi ya Wakimbizi ya Mahama kwa sasa ina VSLAs 15 vilivyotengenezwa hasa na Watu wanaoishi na Ulemavu ila ushiriki uko wazi kwa wanajamii wote.

Wanajamii ikiwajumuisha viongozi, wameunga vyama vya kuleta mapato vilivyoanzishwa na Watu wanaoishi na Ulemavu. VSLAs vyote vikiunganishwa vina wanachama 361 (159 wakiwa nia wa kiume na 202 wakiwa ni wa kike). Kati ya wanachama hawa, 90 ni wa kikundi cha Watu wanaoishi na Ulemavu.

Jumla ya VSLAs 15 vimefungua akaunti kwenye benki.

VSLAs kwa sasa utafutwa na washikadau mbalimbali (kwa mfano srikali ya Rwanda na ubalozi wa kigeni) ili kushiriki katika mashindano, maonyesho na sherehe za kutuza kambi na katika matukio mengine maalum. Katika mikutano ya umma na Siku mbalimbali za Kimataifa, muda fulani hutengewa wakimbizi wanaoishi na ulemavu, familia zao na washiriki ili kushuhudia jinsi VSLAs vimebadilisha maisha yao.

Jamii hushuhudia kuwa wakimbizi wanaoishi na ulemavu pia wana uwezo na nguvu ya kusaidia familia zao na jamii kwa ujumla. 


\section{Desturi ya Kutia moyo kutoka nchi ya Rwanda: Kutambua Manusura na Kuitikia kupitia Ukaguzi}

\section{Matokeo}

- Katika mwaka wa 2017, vituo vya afya vilivyoshirikiswa katika kambi 2 nchini Rwanda vilipata ongezeko la asilimia 300 ya kiasi cha manusura waliotambuliwa na kuunganishwa na utunzi wa kina kwa SGBV katika miezi 2 baada ya kuanzishwa kwa mkakati wa kukagua.

- Katika mwaka wa 2019, katika kambi 5 zilizoshirikishwa nchini Rwanda, watu 326 walitambuliwa kuwa waathiriwa wa SGBV kupitia ukaguzi na kati yao 276 walipewa rufaa kwa huduma za kuitikia SGBV.

- Katika mwaka wa 2019, vituo vya afya vilivyoshirikiswa katika kambi za wakimbizi katika jumla ya nchi 4 (ikijumuisha Rwanda) vilipata ongezeko la asilimia 129 ya kiasi cha manusura waliotambuliwa na kuunganishwa na utunzi wa kina kwa SGBV katika miezi 6 baada ya kuanzishwa kwa mkakati wa kukagua.

- Katika kambi 5 za nchi ya Rwanda ambapo ukaguzi wa SGBV unafanyika, mashirika ya kuitikia SGBV huripoti ongezeko la ghafla katika visa vinavyopokewa, jambo ambalo linafikiriwa kuhusiana na ongezeko la ghafla la utambuzi wa waathiriwa wa SGBV na kuweza kufikia utunzi. Data bora inayokusanywa kutoka kwa wateja na wanaotoa uduma katika kituo cha afya inaonyesha kuwa wanawake na wasichana wanafahamu kuwa wanaweza kuripoti matukio ya SGBV kwa kituo cha afya pamoja na mashirika ya kuitikia SGBV. Hii imesababisha kuongezeka kwa idadi ya visa vinavyotambuliwa vya SGBV.

- Pamoja na nchi ya Rwanda, mkakati huu umetumika na UNHCR na washiriki wenza nchini Sudan Kusini, Uganda na Zambia.

- Kwa pamoja na Baraza ya Idadi ya Watu, UNHCR imefanya mikutano ya kwanza na washirika wanaohusika na elimu, wilaya, walimu na wanafunzi, ili kutayarisha kuanzishwa kwa mkakati wa ukaguzi wa dhulma ya kimapenzi kwa watoto kwenye shule katika kambi za Mahama, Mugombwa na Kigeme. Mkakati huu, ambao pia ulianzishwa na kutathminiwa nchini Kenya na Baraza ya Idadi ya Watu na Hospitali ya Kitaifa ya Kenyatta, haujatekelezwa kwa sababu ya COVID19.

\section{Desturi ya Kutia moyo kutoka nchi ya Rwanda: 'Muungano wa Kijiji wa Kukatiza Kuvumilia Dhulma (ZTVA)}

\section{Matokeo}

- Matokeo ya utathmini wa mkakati wa kijamii wa kuzuia SGBV yameonyesha kupunguka kwa kiasi kikubwa kwa matukio ya: Dhulma ya kudhuru mwili unaotekelezwa miongoni mwa wapenzi (IPV) (wanaume na wanawake), Dhulma ya Kimapenzi kwa IPV (kwa wanaume), Dhulma ya kudhuru mwili miongoni mwa watu ambao sio wapenzi (kwa wanaume na wanawake), na Dhulma za Kimapenzi miongoni mwa watu wasio wapenzi (kwa wanawake).

- Hadi sasa, msaada wa ZTVA umetekelezwa katika takriban vijiji 15 vya wakimbizi nchini Uganda. Matayarisho ya awali (yanajumuisha kutoa mafunzo kwa washirika wanaohusika na utekelezaji, kuhamasisha viongozi wa jamii, na/au Kuundwa kwa Mabaraza ya Washikadau, na kadhalika.) vilevile umefanywa ili hatimaye kuanzisha msaada husika katika makazi ya wakimbizi kule Djibouti, Ethiopia, na Zambia. 


\section{MUHTASARI WA DESTURI}

\section{HARAKATI ZINAZOHUSIANA NA UGONJWA WA COVID}

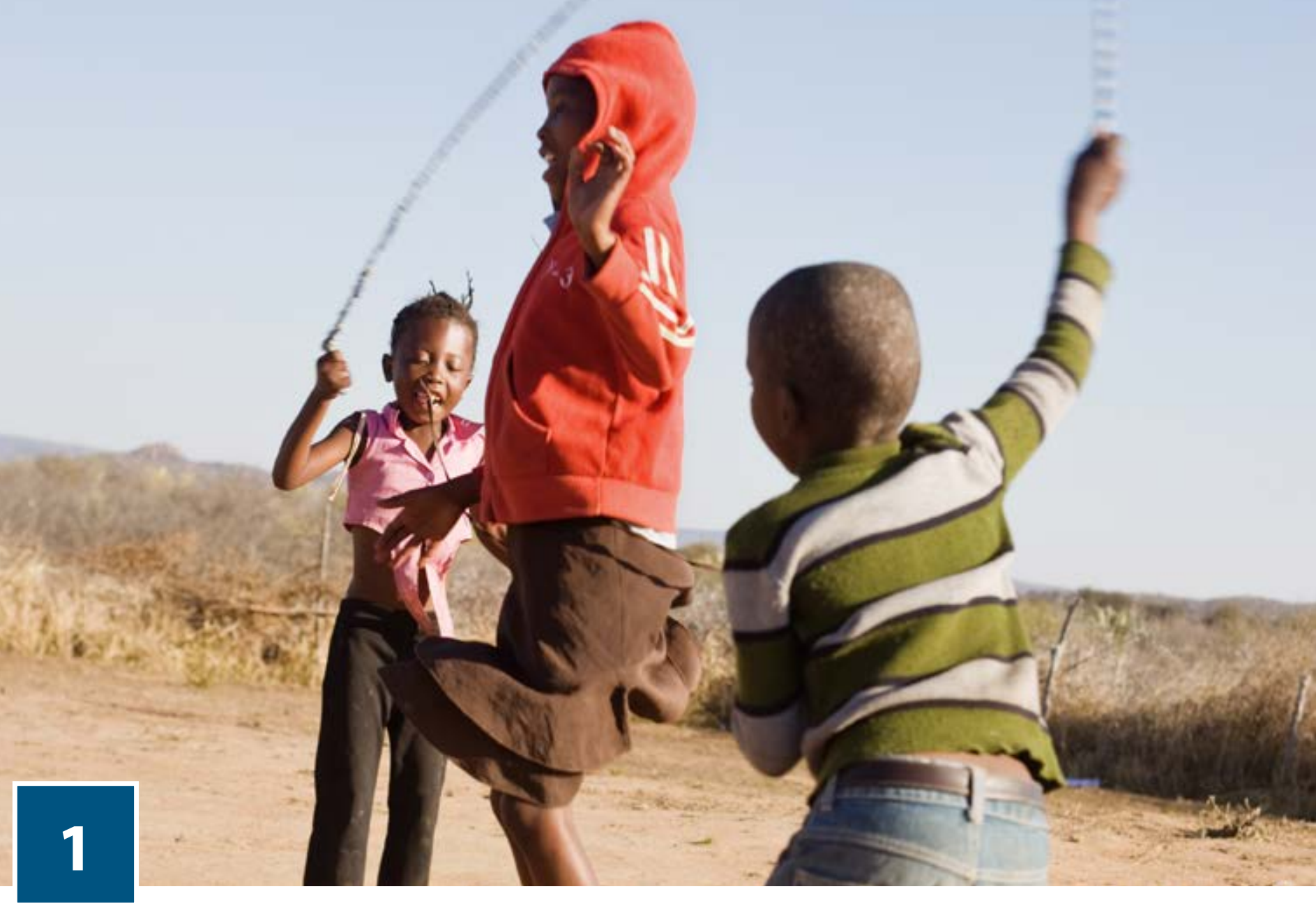

\section{KUWAKINGA WATOTO NA VIJANA}

Desturi Inayojitokeza kutoka Djibouti: Udhibiti wa Visa vya Ulinzi kwa Watoto Mashinani kwa kutumia Wafanyakazi wanaopewa Marupurupu na Wanachama wa Ulinzi wa Kijamii (CBP)

$<$

utambulika kwa visa vya ugonjwa wa COVID- 19 katika kambi za wakimbizi kulielekeza serikali kutekeleza marufuku ambayo ilikatiza kuingia (na kutoka) katika maeneo haya kwa wakimbizi, washiriki wanaotekeleza shughuli sawia na UNHCR. Hili lilisababisha hali ambayo haikutarajiwa ambapo baadhi ya watoto walitenganishwa na wazazi wao (kwa mfano, ambapo wazazi walikuwa wamesafiri nje ya kambi kwa ajili ya kazi au kupata utunzi wa kimatibabu).

Desturi hii inayojitokeza hivyo inajumuisha UNHCR na washiriki wenza wanaotekeleza shughuli mashinani pamoja na wafanyakazi wanaopewa marupurupu na wanachama wa CBP wanaofanya kazi ya kuwalinda watoto katika kambi za wakimbizi kufuatilia udhibiti wa visa vya watoto waliotenganishwa, wasio na wanaoambatana nao, watoto walio hatarini na waliotelekezwa au kuachwa. 
Vipengele hususa vya mkakati huu ni matembezi ya nyumbani, kuhamasisha ufahamu na kukusanya data ya udhibiti wa visa vya ulinzi wa watoto, kama inavyoelezwa hapa:

\section{Matembezi ya nyumbani}

- Kukadiria mahitaji ya watoto na hali ya maisha katika makazi ya wakimbizi ikijumuisha makazi, usaidizi wa kifedha, usaidizi wa chakula na usaidizi wa kimatibabu.

- Kutambua watoto walio na mahitaji maalum na mahangaiko ya ulinzi, kama vile wahasiriwa na dhulma ya kimwili au kijinsia, kuozwa mapema na ujauzito.

- Kutambua watoto waliotelekezwa, walioachwa, waliotenganishwa, wasio na watu wanaoandamana nao na yatima.

\section{Kuhamasisha ufahamu}

- Kuhamasisha ufahamu wa jamii kuhusu haki za watoto na kuhusu hali ambazo zinaelekeza watoto kuhitaji ulinzi kama vile dhulma za kingono na kimwili kwa watoto, kuajiriwa kwa watoto na ndoa za mapema.

- Kuhamasisha ufahamu wa jamii kuhusiana na hatua za kujilinda na COVID- 19 za kijamii.

\section{Kukusanya data}

o Kuratibisha idadi ya watoto walio kwenye kambi ambao wametenganishwa, mayatima, walioachwa au wasio na watu wanaoambatana nao.

- Kutoa ripoti za kila wiki kuhusu shughuli zinazofanya katika kambi na hali haswa ilivyo kambini.

- Kutambua visa vya ulinzi wa watoto vinavyohitaji usaidizi mara moja na kuviripoti.

- Kufanya utafiti katika nyumba ili kukadiria hali ya kiakili na kijamii ya watu wa nyumba zile (ikijumuisha watoto) na athari za vikwazo vya COVID- 19 kwa maisha ya kila siku ya wakimbizi.

\section{Matokeo}

Jumla ya watoto manusura 41 walitambuliwa kutumia mkakati huu katika kipindi cha kwanza cha miezi 4 kufuatia kuanza kwa ugonjwa wa COVID- 19. Manusura hawa wameorodheshwa katika mradi wa udhibiti wa visa unaoendelea.

Washiriki: International Children's Action Network

Kwa habari zaidi, wasiliana na:

Laura De Somer: desomer@unhcr.org

Arnaud Ntahompagaze: ntahompa@unhcr.org

Helene Chege Wangui: helen.wangui.c@gmail.com

\section{Desturi Inayojitokeza from Tanzania: Redio ya Jamii ili kutoa Mafunzo wakati wa ugonjwa wa COVID-19}

$\gamma$

atika kambi za wakimbizi nchini Tanzania, mtazamo wenye njia mbalimbali unatumika ili kuhakikisha kuendelea kwa masomo wakati wa mlipuko wa ugonjwa wa COVID- 19. Mtazamo huu unahusisha matumizi ya mradi wa mafunzo ya mbali kupitia redio ya jamii, ikifungamanishwa na kutayarishwa kwa pakiti za masomo zinazowasilishwa nyumbani kwa wanafunzi.

Kikundi cha Utendakazi cha Mafunzo kwa Wakimbizi kilifanya mikutano na serikali na kupata idhini ya kutengeneza na kupeperusha mpango wa masomo kupitia redio utakaotimiza mahitaji ya wakimbizi. Baada ya kupata idhini, EWGkilianza kufanya kazi na Redio ya Kwizera katika kupanga upeperushaji. Mchakato wa kupanga ulihusisha kushughulikia vikwazo vya kifedha na kiteknolojia. Kwa vile kuna uhaba wa kuweza kufikia mitambo ya redio katika kambi za wakimbizi, Kikundi cha Utendakazi cha Mafunzo kwa Wakimbizi kinashughulika ili kuweza kununua na kusambaza vifaa vya redio vinavyotumia nishati ya jua manyumbani kwa wakimbizi. Washiriki wa UNHCR katika masomo pia wanatengeneza bajeti zao upya na kubadili mitazamo yao ili kukidhi mahitaji yanayojitokeza.

Isitoshe, vifurushi ya masomo vya kuenda navyo nyumbani, ikijumuisha nakala za vitabu vya mafundisho vinavyolingana na mtaala vinatayarishwa ili kutumika katika mafunzo ya nyumbani huku vikilenga hasa wanafunzi wa vyuo vya sekondari.

\section{Washiriki:}

Kikundi cha Utendakazi cha Mafunzo kwa Wakimbizi

Redio ya Kwizera

Kwa habari zaidi, wasiliana na:

Tamar Joanian: joanian@unhcr.org 


\section{Desturi Zinazojitokeza kutoka Uganda: Mabalozi Watoto wa COVID- 19}

M lipuko wa COVID- 19 ulilazimu kuanzishwa kwa mafunzo kwa jamii ili kuhakikisha ufuataji wa kanuni za Wizara ya Afya, ikijumuisha uoshaji wa mikono, kukaa nyumbani, na kukaa mbali na mwenzako. Hivyo, shirika la World Vision lilianzisha mpango wa uhamasishaji wa jamii kwa ushirikiano na viongozi wa jamii, ikijumuisha wanachama wa Kamati ya Ulinzi kwa Watoto na viongozi wa makundi ambao walisambaza sabuni kwa manyumba yaliyo hatarini ili kutumia katika kuosha mikono. Wakati wa kusambaza bidhaa katika manyumbani, ilionekana kuwa watoto walifanya mzaha kwa waliokuwa wakisambaza kwa kupaza sauti wakisema neno 'corona.' Kati ya makundi ya usambazaji bidhaa, ni 'William,'mtoto mkimbizi wa kutoka Sudan Kusini, ambaye alikuwa mwanachama wa klabu ya usafi iliyoanzishwa na shirika la World Vision shuleni, ndiye pekee alipendeza watoto na kuwa walionekana kupenda kumsikiliza. Hivyo basi, watoto wakakuwa kipengele muhimu katika mpango huu. Umuhimu wa kuwashirikisha katika mpango huu ulitiliwa mkazo na vikwazo vya kutoka na kuingia vilivyowekwa wakati wa mlipuko, ambavyo vilikatiza shughuli za kila siku za watoto na kuwafanya wasiwe na mahali pa kuenda au kucheza na kuingiana na marafiki zao. Hali hii iliwapa wazazi na watunzi msongo mipya ya mawazo ambao iliwabidi wakati mwingine kukosa kuenda kazini au kutafuta mbinu mpya ya kuwapatia watoto wao utunzi.

Mkakati huu ulilenga kuwawezesha watoto kuwasiliana na wenzao kwa njia bora katika hali mpya iliyoletwa na ugonjwa wa COVID- 19 na kuhusika katika kuzingatia hatua za kujikinga. Mkakakati huu ulianza na William, mwanachama wa klabu ya usafi aliyechukuliwa ili kusaidia akiwa kama balozi wa mkakati huu. Jukumu lake lilikuwa ni kukuza ufahamu kwa watoto wengine katika makazi, alipewa kipaza sauti ili kuwasilisha jumbe kwa vijiji mbalimbali huku akiwahamasisha watoto wenzake kuhusu njia za kujikinga ugonjwa wa COVID- 19. Tangu wakati huo, watoto 19 mabalozi (watoto 9 wa kike na 10 wa kiume) wameshiriki katika vipindi vya siku 2 vya mafunzo kuhusu COVID- 19 vilivyotekelezwa na wahudumu wawili wa afya na mfanyakazi mmoja kutoka kwa shirika linaloendeleza shughuli hii inayolenga mawasiliano katika jamii. Vipindi hivi vya mafunzo hujenga uwezo wa watoto kueneza habari zinazohusiana na ugonjwa wa COVID- 19 kwa watoto wenzao na kwa jamii. Sawia na William, watoto waliopokea mafunzo upatiwa vipaza sauti ili kuwafikia watoto wenzao katika maeneo wanayoishi. Pia, wanapatiwa vipeperushi vilivyo na jumbe zinazowafaa watoto zilizoandikwa na Wizara ya Afya na kuchanganuliwa na watoto hawa kama sehemu moja ya mafunzo.

\section{Matokeo}

Kufikia mwezi Juni 2020, watoto 948 (watoto 458 wa kike na 490 wa kiume) walikuwa wamefikiwa na jumbe kuhusu ugonjwa wa COVID- 19 kutoka kwa watoto mabalozi waliokuwa wamepokea mafunzo wakati wa vipindi vya uhamasisho vya nyumba hadi nyumba. Kupitia kushiriki kwa katika mkakati huu, watoto hawa waliona na kuripoti kukosekana kwa sabuni katika familia nyingi zilizofikiliwa na kuomba kufanyika kwa shughuli ya usambazaji wa sabuni zaidi ili kuendeleza usafi wakisaidiwa na Kamati za Ulinzi kwa Watoto.

Washiriki:

Shirika la Kimataifa la World Vision

Kwa habari zaidi, wasiliana na:

Veronique Njo: njo@unhcr.org

Brenda Adrako Madrara: Brenda_Madrara@wvi.org; abitimorenda@gmail.com 


\section{Mfano wa Kisa kutoka nchi ya Sudan Kusini: Uhamasisho wa Wanafunzi wa Kutuma jumbe kuhusu ugonjwa wa COVID- 19 katika Maeneo ya Mjini}

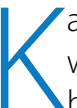
atika makazi ya Wakimbizi ya Gorom mjini Juba, wanafunzi wanofaidika na udhamini kutoka kwa UNHCR walijitolea kusaidia jamii zao katika kueneza habari kuhusu jinsi ya kujikinga na ugonjwa wa COVID- 19. Wanafunzi hawa waliojitolea walipewa mwelekeo kuhusu habari muhimu za kujikinga za Shirika la Afya Ulimwenguni na wakagawanywa katika makundi na kuongozwa na wahudumu wa afya katika jamii au wanaohamasisha usafi. Makundi haya hutekeleza kampeni zenye habari zinazowalenga watu manyumbani, kuonyesha desturi za kimsingi za usafi kulingana na jumbe zinazopendekezwa za Habari, Mafunzo na Mawasiliano. Makundi haya ya wanafunzi pia huwapatia rufaa visa vya kimatibabu hadi kwa washiriki wanaoshughulikia afya.

Washiriki: mbalimbali

Kwa habari zaidi, wasiliana na:

Tamar Joanian: joanian@unhcr.org

\section{Desturi Zinazojitokeza kutoka Sudan Kusini: 'Kila mtu Anaweza Kufanya Mabadiliko; Kila Tendo ni la Muhimu'}

Yarik

atika kambi ya wakimbizi ya Doro, kila kijiji kina mwakilishi wa vijana (Mwenyekiti) ambaye hushiriki katika Kamati ya Vijana ya Kambi. Kabila nane tofauti zimetambuliwa kwa wakimbizi hawa huku kila moja likiwa na lahaja zake tofauti. Kwa vile kuna hitaji la uhamasisho kuhusu COVID- 19 na desturi nzuri za usafi, uanuawai huu huwa bora, hivyo kuwezesha mawasilianokupitia lugha mbalimbali za wenyeji. Kuweka jumbe muhimu za ugonjwa wa COVID- 19 katika muktadha unaoafikiana na kambi ya eneo hususan kunahitajika ili kufanikisha jambo hili.

Katika mkakati huu ulioongozwa na vijana, watu wachanga katika kambi za wakimbizi wamejihusisha na uhamasisho wa jamii kuhusu ugonjwa wa COVID- 19. Wenyekiti wa Vijana wameungana na kutunga nyimbo nane kuhusu ugonjwa wa COVID- 19 kutumia lugha zao. Nyimbo hizi zinalingana na jumbe kuhusu COVID- 19 ambazo zimepitishwa na Shirika la Afya Ulimwenguni. Kikundi hiki cha vijana hutembelea jamii moja hadi nyingine, huku wakiwahamasisha watu wa jamii kuhusu ugonjwa wa COVID- 19, na kuimba nyimbo zilizotafsiriwa kulingana na lahaja inayozungumzwa katika eneo ndogo hususa.

Kikundi hiki kinahakiksha kimetimiza umbali unaohitajika kutoka kwa mtu mmoja hadi mwingine katika maonyesho yao yote pamoja na uoshaji mikono unaostahili.

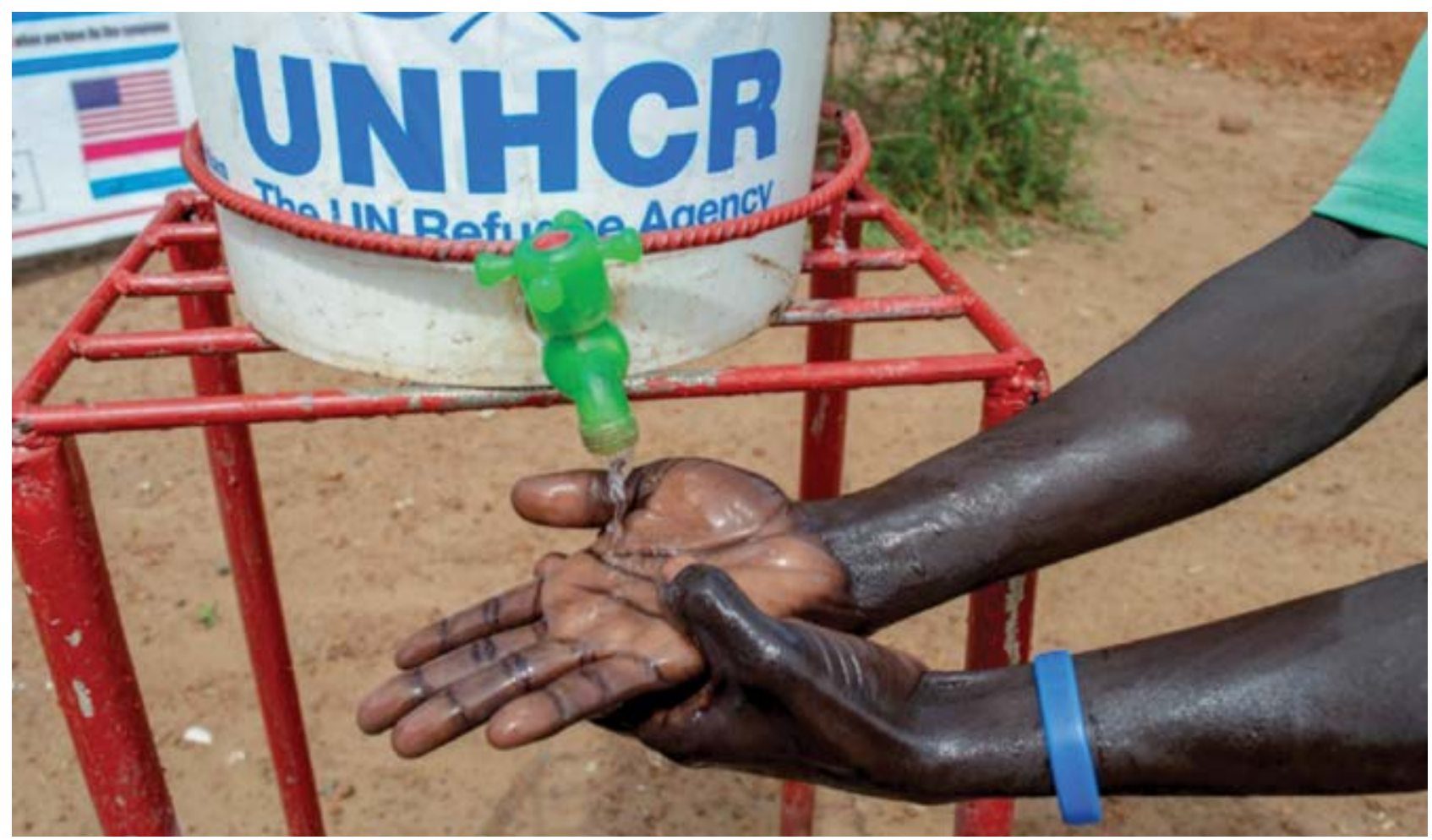



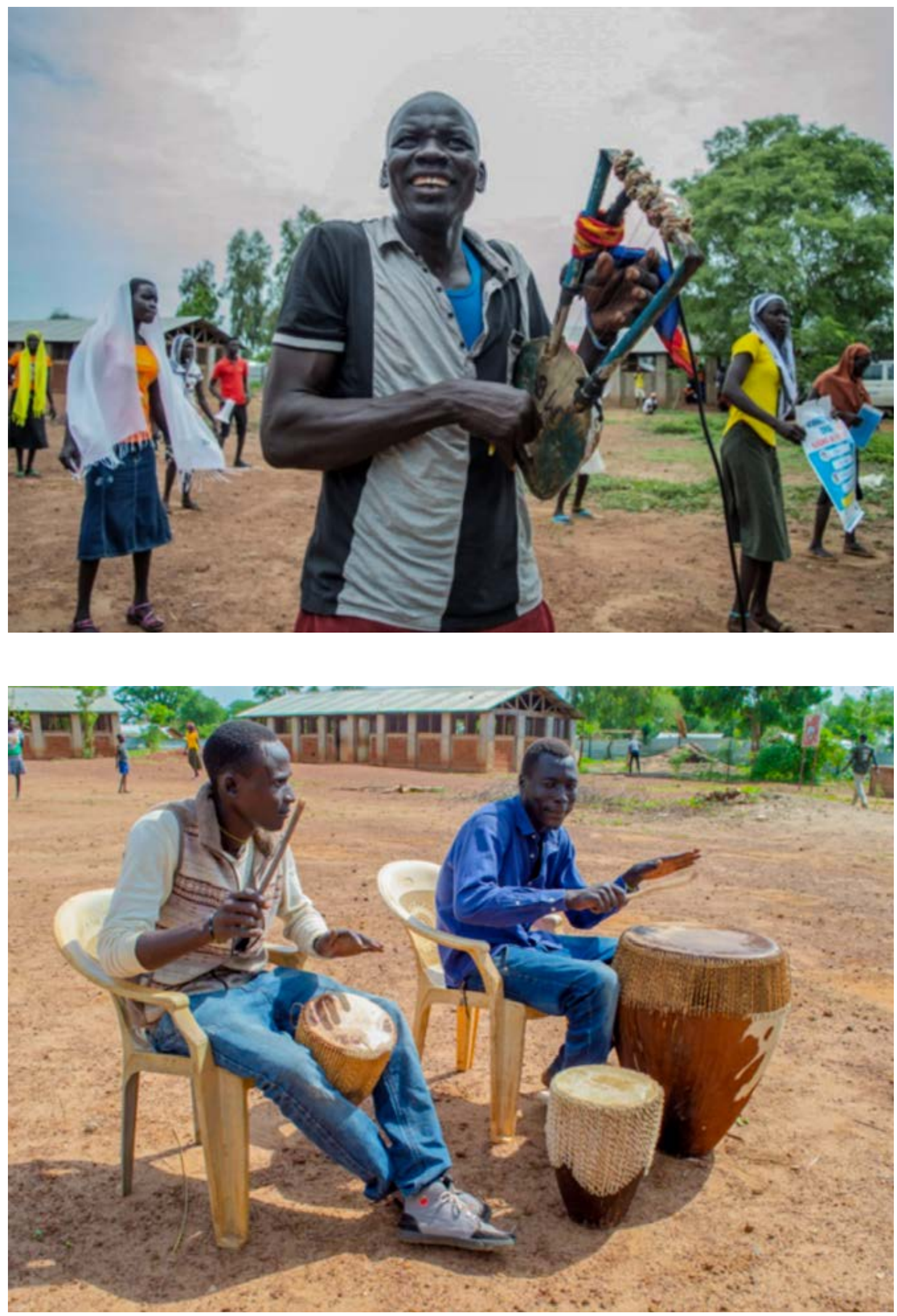


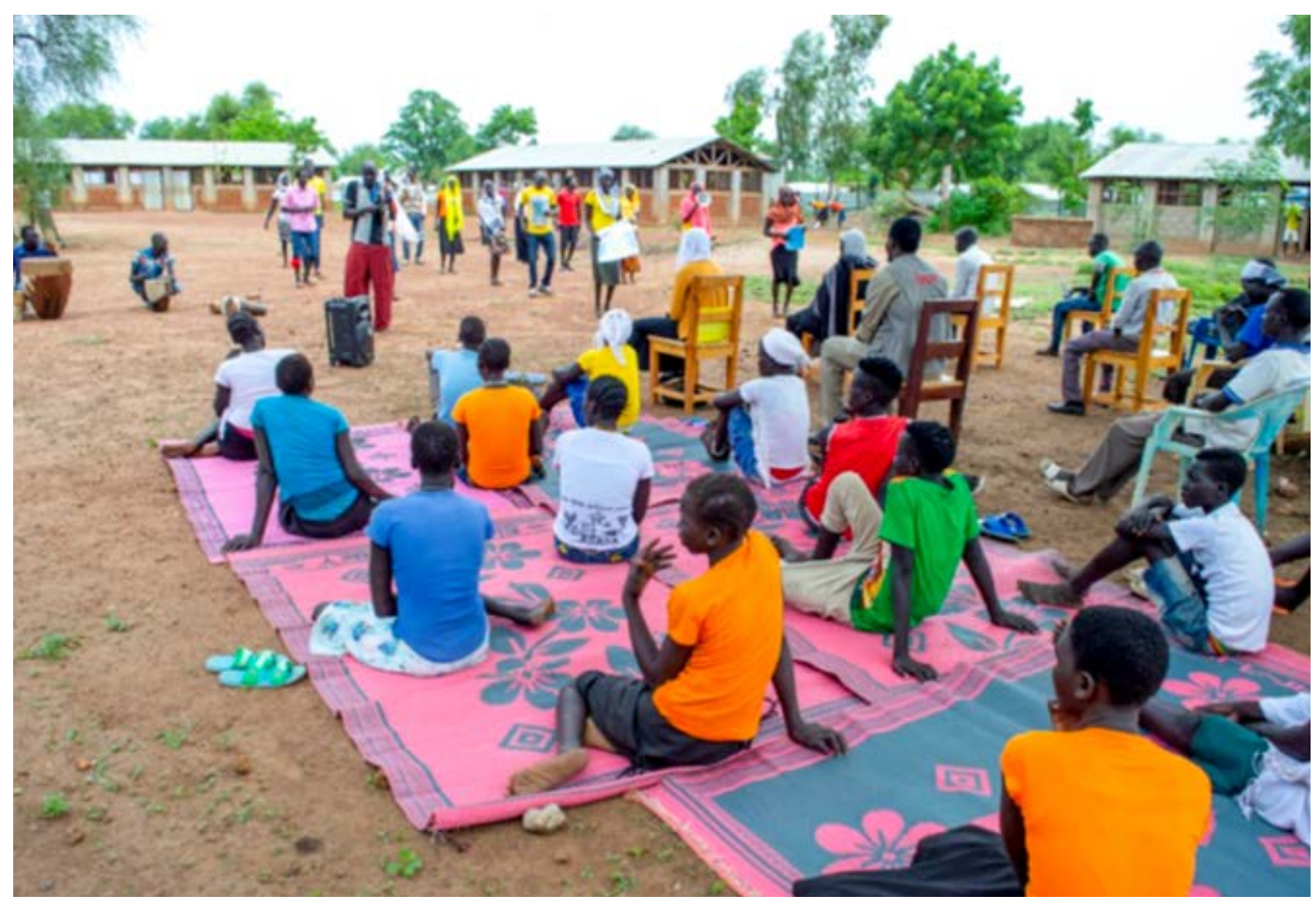

Katika kila tamasha, kikundi cha wanaojitolea huelekeza watazamaji katika maeneo ya uoshaji mikono. Bango kubwa lenye maudhui kuhusu COVID- 19 huwekwa nyuma ya maeneo haya ya kuosha mikono. Wakati watu wa jamii wanapanga foleni, kila mmoja anaelekezwa kusimama takriban umbali wa mita moja mbali na mwenzake. Ili kuhakikisha umbali huu haujakiukwa, wanajamii wanahimizwa kusimama katikati ya miviringo iliyochorwa chini ardhini. Mtu mmoja aliyejitolea husimama kando ya kila eneo la kuosha mikono ili kuwaelekeza wanawake, wanaume na watoto kuhusu mbinu zinazopendekezwa za kuosha mikono.

Viti vya watazamaji zimewekwa umbali wa mita moja kutoka kwenye kiti kimoja hadi kingine na kila mtu anayependelea kuketi kwenye mikeka hushauriwa kuakikisha umbali huu umetiliwa maanani. Wakati viti vyote (viti na mikeka) vimejaa, maonyesho yanaanzishwa. Nyimbo hizi hujumuisha mafumbo yanayotumika katika eneo fulani na kuangazia desturi nzuri kama vile kuepukana na salamu za mkono na kukaa umbali unaohitajika kutoka kwa mtu mmoja hadi mwingine. Baada ya nyimbo kadhaa, kikundi hiki hutoa hotuba zilizo na maudhui ya jinsi ya kujikinga na COVID- 19.

Kamati ya Vijana huratibu idadi ya maeneo ambayo imetembelea, idadi ya vipindi vya maonyesho vilivyofanyika na idadi ya watu waliofikiwa na ujumbe huu. Katika mwezi wa Julai 2020, kupitia mtazamo wa sekta mbalimbali, chini ya mpango wa Dhulma za Kimapenzi na Kijinsia, miundo ya kijamii ya Dhulma za Kimapenzi na Kijinsia (Wasiamizi wa Ulinzi, Wanaharakati wa kikundi cha SASA!, watetezi wa Dhulma za Kimapenzi na Kijinsia) walijihusisha na vipindi vya uhamasisho na kusambaza habari kuhusu jinsi ya kujikinga Dhulma za Kimapenzi na Kijinsia ambazo zimefungamanishwa na jumbe kuhusu jinsi ya kujikinga COVID- 19. Jumla ya watu 4,899 wa jamii (watu 2,092 wa kiume na 2,807 wa kike) walifikiwa na habari hii.

Kikundi hiki cha vijana kilianzisha mkakati huu chenyewe ila kinahitaji msaada kutoka kwa mashirika ya kutoa misaada ili kuendeleza shughuli zao. Mikakati ya kijamii kama hii inaweza kuwa ni jukwaa lenye ushawishi katika kufikisha jumbe muhimu zilizolinganishwa na muktadha na mila ya eneo husika.

\section{Washiriki:}

Tume ya Masuala ya Wakimbizi

Baraza la Wakimbizi nchini Denmark

\section{Kwa habari zaidi, wasiliana na:}

Dr. Gebrewold Petros Yohannes: petros@unhcr.org

Thomas John Hither, Mwenyekiti, Kamati ya Vijana, Kambi ya Wakimbizi ya Doro

Alor Kuol, Naibu wa Meneja wa Kambi ya DRC, Kambi ya Wakimbizi ya Doro 


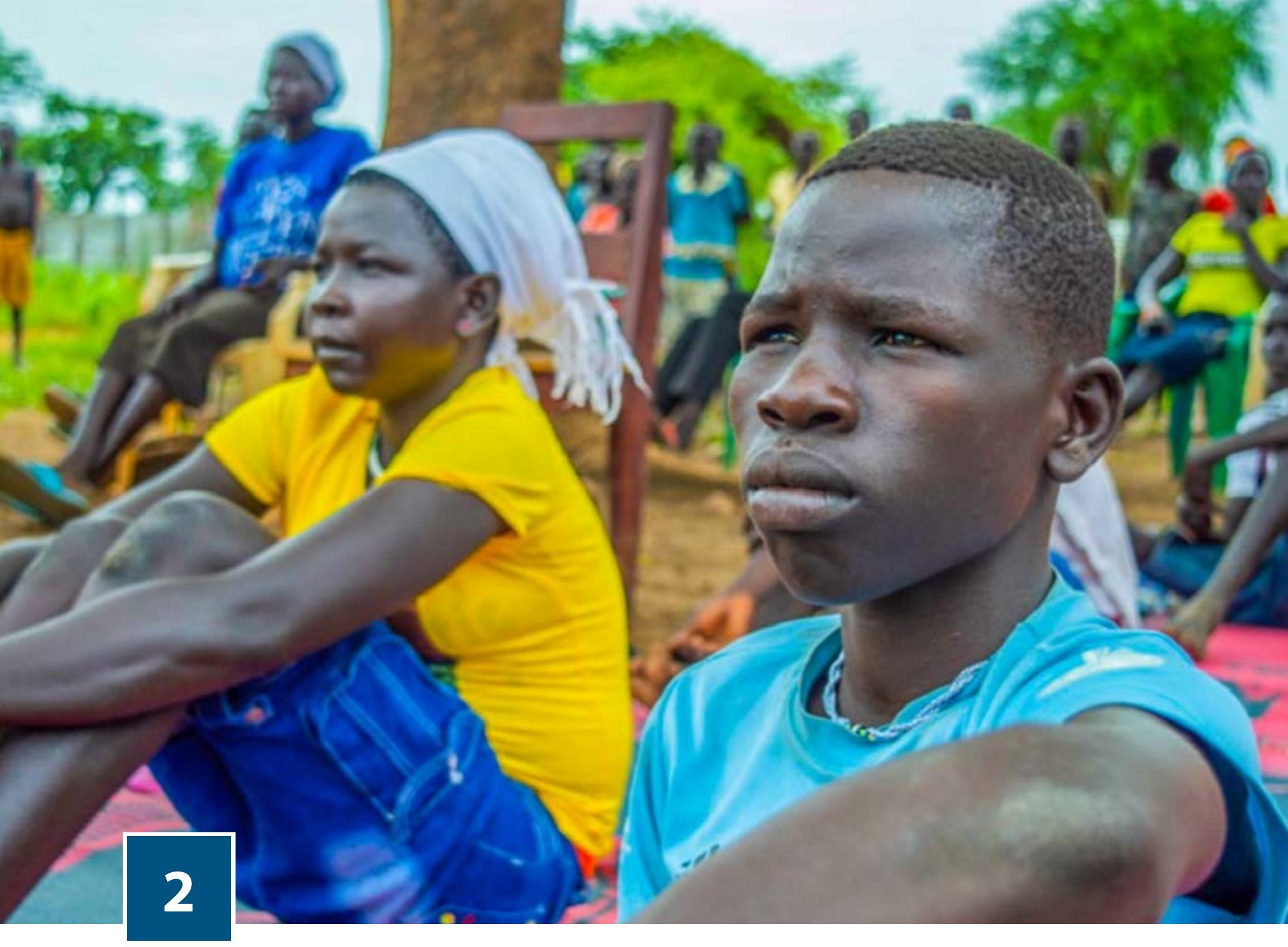

\section{ULINZI WA KIJAMII}

\section{Desturi Inayojitokeza kutoka nchini Djibouti: Huduma za Mashinani za Jamii}

$\mathrm{H}$ atua za kuzuia COVID- 19 nchini Djibouti ziliwaelekeza wafanyakazi wa UNHCR na washiriki wake katika utendakazi kuanza kufanya kazi kwa kutumia simu. Hili lilikuwa na athari katika kufikilia huduma za ulinzi kwa Watu Husika.

Mkakati uliopo ulitokana na hitaji la kudumisha uendelevu wa huduma za ulinzi na kuwahusisha wakimbizi pamoja na wanaotafuta hifadhi. Mkakati huu unajumuisha kuanzishwa kwa huduma ya simu watakayotumia wakimbizi na wanaotafuta hifadhi. Huduma hii ya simu ilifungamanishwa na makundi mawili ya mtandao wa WhatsApp katika kambi za wakimbizi - kikundi kimoja kikiwa ca viongozi wa jamii na kikundi kingine kikiwa cha wahudumu wa afya walioko katika jamii.

Nambari ya huduma hii ya simu inasambazwa kote (kupitia mabango yenye habari yaliyoandikwa katika luga ya Oromo,Somali, Kifaransa, Kiarabu na Kiingereza) ili kufahamisha idadi kubwa ya wakimbizi na wanaotafuta hifadhi (wanawake, wanaume, wasichana na wavulana) kuhusu kuwepo kwa huduma hii. Huduma hii inapatikana kila siku kutoka saa 2 asubuhi hadi saa 11 jioni huku makundi ya mtandao wa WhatsApp yanafanya kazi saa 24 kila siku. Viongozi wa jamii za wakimbizi wanapewa simu iliyolipiwa ada/muda wa maongezi ili kudumisha mawasiliano na wanajamii, wafanyakazi wa ulinzi wa UNHCR na baina yao wenyewe.

Watu wenye matatizo ya kiafya na mahitaji mengine ya dharura hupewa rufaa na viongozi wa jamii hadi kwa wahudumu wa afya ambao hutoa utunzi na kuwasaidia katika kuwaelekeza kwa washiriki wanaohusika (washiriki wenzao wa kitaifa na/ au washiriki wa UNHCR wanaoendeleza shughuli) kulingana na hatua za usalama zilizowekwa. Wahudumu wa afya katika 
jamii pia wanapokea mafunzo ya jinsi wanaweza kushughulikia matatizo ya kiafya yanayohusiana na COVID- 19. Simu zinazopigwa kwenye nambari ya simu ya msaada zinaratibiwa pamoja na huduma zinazotolewa kutokana na simu hizo.

Mkakati huu umesaidia UNHCR katika: kudumisha mawasiliano na miundo ya jamii; kudumisha shughuli za udhibiti wa visa; na kusikiliza na kuwaongoza wakimbizi na wanotafuta hifadhi walio na malalamiko na matatizo yaliyozidishwa na janga hili.

Washiriki: haihusiki

Kwa habari zaidi, wasiliana na:

Laura De Somer: desomer@unhcr.org

Dr. Tongna Alain Rodrigue Zoure: zoure@unhcr.org

Oubah Abdi Hassan: abdihaso@unhcr.org

\section{Mfano wa Kisa nchini Ethiopia: Makundi ya mtandao wa WhatsApp ya Viongozi wa Jamii na Wakimbizi wa Kujitolea Kuwasilisha huduma mjini Addis Ababa}

ngawa wakimbizi nchini Ethiopia wamewekwa katika kambi za wakimbizi, makundi mbalimbali ya wakimbizi pia yanaishi katika maeneo ya mjini ya mji wa Addis Ababa. Baadhi ya wakimbizi huhamia mjini Addis Ababa baada ya kuhamishwa kutoka kambi za wakimbizi kutokana na sababu za kiafya, usalama, Dhulma za Kimapenzi na Kijinsia na sababu zinginezo zisizoweza kutatuliwa katika kambi hizi. Wakimbizi wa asili ya nchi ya Eritrea ndio wengi zaidi katika idadi ya wakimbizi wanaoishi mijini. Wengi wao huishi katika mji wa Addis Ababa chini ya Sera ya Kuishi nje ya Kambi, mpango maalum na Vserikali ya nchi ya Ethiopia, kuzingatia mila na misingi ya kihistoria na mahusiano kati ya nchi ya Ethiopia na Eritrea.

Hatua mbalimbali za kujikinga COVID- 19 zilizoanzishwa mjini Addis Ababa ikijumuisha kufungwa kwa kituo cha mapokezi na kuwekwa kwa vikwazo katika huduma za ana kwa ana, zilizuia mawasiliano ya UNHCR na jamii za wakimbizi wanaoishi katika mji wa Addis Ababa. Mji wa Addis Ababa kwa kawaida una mtandao mwema wa simu na intaneti na wakimbizi wanaoishi mji huu wanafurahia muunganisho huu mzuri. Asilimia inayofikia 94 ya nyumba za wakimbizi wanaoishi mji wa Addis Ababa wanaweza kufikilia simu ya rununu.

Kwa vile njia za mawasiliano za kawaida kwa jamii za wakimbizi (katika kituo cha mapokezi, mikutano ya ana kwa ana) zilizidi kuzuiliwa vikali kwa sababu ya vizuizi vya COVID- 19, mbinu mbadala za mawasiliano ziliangaziwa. Kwa vile mji wa Addis Ababa una msambao mwema wa simu za rununu, njia za mawasiliano ya kidijitali zilianzishwa, ikijumuisha makundi ya mtandao wa WhatsApp ya UNHCR na viongozi wa jamii ya wakimbizi na wanaojitolea kutekeleza shughuli.

Mkakati huu ulilenga kuhakikisha mawasiliano bora ya pande mbili kati ya UNHCR na jamii za wakimbizi kuhusu mada mbalimbali ikijumuisha habari kuhusiana na uhamisho, jumbe kutoka kwa Wawakilishi wa UNHCR kwa wakimbizi, kupatikana kwa huduma, nambari za huduma ya simu, habari kuhusu hatua za kujikinga COVID- 19 na ufafanuzi zaidi kuhusu habari zisizo sahihi.

UNHCR ilianzisha makundi matatu ya WhatsApp ya viongozi wa jamii ya wakimbizi (wanachama 15-20 kwa kila kikundi), wanaowakilisha raia wakuu katika mji wa Addis Ababa, na kikundi kimoja cha Wanaojitolea Kutoa huduma kwa Wakimbizi. Wafanya kazi wa UNHCR (Kikundi cha Mjini) wanatumika kama wasimamizi wa vikundi vyote vinne, huku wakituma habari husika kwenye makundi na kujibu maswali.

Wanachama wa makundi haya ya WhatsApp wanahitajika kufuata kanuni zilizowekwa ikijumuisha kutojadili visa wala kusambaza habari nyeti au za kibinafsi kuhusu wakimbizi.

Makundi haya ya WhatsApp yanakubalisha uwezo wa kufikilia habari husika kwa viongozi wa wakimbizi ambao hupewa jukumu la kuwasilisha habari hii kwa wanajamii ikijumuisha makundi ya watu wenye mahitaji maalum. Mawasiliano ya pande mbili ni chombo cha kukuza uwezo na kuboresha ushirikiano kati ya viongozi wa wakimbizi na UNHCR.

Washiriki: haihusiki

Kwa habari zaidi, wasiliana na:

Ota Hlinomaz: hlinomaz@unhcr.org

Nunu Aliyi: aliyi@unhcr.org 


\section{Mfano wa Kisa kutoka Kenya: Kamati ya Ulinzi wa Jamii}

$\mathrm{M}$ lipuko wa ugonjwa wa COVID- 19 uliongeza hitaji la kuhakikisha kuweza kupata habari kwa wakati unaofaa, habari inayofaa na sahihi katika lugha zote husika kwa Watu Husika pamoja na kuweza kufikilia mawasiliano na udhibiti mahususi wa ulinzi wa visa kwa watu walio katika hatari iliyoongezeka (kwa mfano, watu wa umri wa juu, watoto, wanarika, Watu wanoishi na Ulemavu, watu wa kikundi cha LGBTI, na watu walio wachache wa kabila au dini fulani). Jamii ilipendekeza kutengenezwa kwa kamati itakayofanya kazi pamoja na kamati za sekta zingine. Katika kuitikia pendekezo hili, na kuangazia mtazamo wa AGD, UNHCR ilianzisha kamati ya ulinzi ya wanachama 36 ambapo wanachama walifunzwa kuhusiana na kanuni za kimsingi za Kutoa Ulinzi, Usiri, Njia ya Kutoa Rufaa na kukinga/kupunguza mlipuko wa COVID- 19.

Baada ya kuanza kwa mlipuko wa COVID-19, hudhurio la kambini/kuwepo kwa UNHCR na washiriki ilishuka chini hata ingawa kazi iliendelea mashinani kupitia huduma ya simu, utumaji wa jumbe nyingi, matumizi ya mtandao wa WhatsApp na maonyesho ya mazungumzo kwenye redio. Hata hivyo, wakimbizi walitaka habari zaidi kutoka kwa UNHCR wakidai kuwa hawangeweza kutegemea habari wanayopata kutoka kwa viongozi wao tu.

Kipengele kikuu cha mkakati huu kinahusisha kutumia mitandao iliyopo ya wanaojitolea kutoa huduma na makundi ya wanawake, watoto na makundi mengine yaliyotengenezwa na kuongozwa na Watu Husika ili kutoa habari muhimu kuhusu shughuli muhimu za kisekta na mahitaji ya ulinzi pamoja na kuhakikisha njia za kutoa rufaa zinadumishwa huku zikitoa ulinzi kwa jamii na msaada wa kisaikolojia. Kamati ya Ulinzi kwa Jamii hutengeneza jukwaa kwa UNHCR kutumia mitandao hii. Kamati hii ya wanachama 36 inapokea habari kutoka kwa washiriki na kueneza hadi kwa jamii kijumla. Kamati hii pia hufanya kazi na watekelezaji sheria na shirika la usimamizi wa kambi ili kuhakikisha kuwa visa vyote vinavyowasili vinawekwa kwenye kituo cha karantini sawia na Kamati zingine za kisekta za wakimbizi.

Majukumu mengine ya kamati hii ni:

- Kufanya matembezi ya nyumba hadi nyumba au ya nyumbani kwa familia na kutoa habari ya kimsingi kuhusu huduma zinazotolewa na masuala mengine yanayojitokeza.

- Kuwatambua kwa usalama wakimbizi walio hatarini zaidi wanaohitaji msaada wa dharura, na kuwapa rufaa hadi kwa UNHCR na washiriki au kitengo/sekta husika ili kupata mwitikio wa haraka.

- Kuanzilisha mitandao pamoja na wanaojitolea kutoa huduma kwa wakimbizi, wafanyakazi na miundo, viongozi wa vitengo na kijumla, polisi wa Kenya ili kuwezesha kutoa rufaa.

- Kutekeleza uenezaji wa habari katika jamii kuhusu kujlinda na kujikinga dhidi ya kuambukizwa virusi vya Corona.

- Kufanya ufuatiliaji wa mara kwa mara wa ulinzi wa vitengo kupitia viongozi wa vitengo hivyo ili kuweza kukusanya habari na kuwajulisha kwa haraka kuhusu masuala yanayojitokeza kama vile afya, dhulma za kimapenzi na kijinsia, ulinzi kwa watoto, makazi, mahitaji ya kimsingi na kwa malengo mengine yoyote.

- Kutoa habari za kila siku na ripoti kwa UNHCR kila siku ya Alhamisi.

UNHCR na Barazaya Wakimbizi ya Norway hutoa ada ya uda wa maongezi kupitia simu kwa wanachama wa kamati hizi ili kuhakikisha mawasiliano ya pande mbili na kuwezesha kutoa ripoti za kila juma jinsi hali ilivyo.

Washiriki:

Baraza ya Wakimbizi ya Norway

Sekretariati ya Masuala ya Wakimbizi

Kwa habari zaidi, wasiliana na:

Lina Koki: koki@unhcr.org

Nicholas Midiwo: midiwo@unhcr.org 


\section{Mfano wa Kisa kutoka Kenya: Matumizi ya Mtandao wa Utumaji ujumbe wa WhatsApp na Wafanya kazi wa Jamii ya Wakimbizi katika mpango wa WASH}

I izuizi vya safari wakati wa mlipuko wa COVID- 19 kulielekeza UNHCR na shirika la CARE kuwategemea Wafanyakazi wa Jamii ya Wakimbizi(RCWs) ili kutoa msaada wa ufuatiliaji kwa shughuli za mpango wa WASH katika jamii za wakimbizi. RCWs hufuatilia shughuli katika visima na katika jamii kwa ujumla. Kuanza kwa mlipuko wa COVID- 19 kulisababisha hitaji la matumizi ya mfumo wa mashinani ili kuwezesha mawasiliano ya habari za ufuatiliaji, ikijumuisha kupiga na kutuma picha zinazoonyesha uendelevu wa shughuli, na kuripoti malalamiko ya dharura kama vile mifereji inayovuja na mabomba yaliyopasuka. Mtandao wa utumaji jumbe wa WhatsApp umetumika kwa kusudi hili. Matumizi ya mtandao huu umehakikisha kuwa malalamiko yameripotiwa na kushughulikiwa kwa upesi, na kuwa ripoti za uendelevu zinazidi kupokelewa na kuwasilishwa katika ofisi za shirika la CARE linalofanya mpango wa WASH. RCWs na viongozi wa kambi wana nambari za mawasiliano za maafisa wa mpango wa WASH katika shirika la CARE na UNHCR na wanaweza kuwasilisha malalamiko wakati wowote saa za mchana na hata usiku kutumia mtandao huu.

Washiriki:

Shirika la CARE

Kwa habari zaidi, wasiliana na:

Osman Yussuf Ahmed: ahmedos@unhcr.org

\section{Desturi ya Kutia moyo kutoka nchi ya Rwanda: Kikundi cha Kufanya kazi ya Usafi katika Jamii na Jitihada za Pamoja za Jamii za Kukinga COVID- 19, Ikiwajumuisha Wanamuziki wa Rwanda}

M lipuko wa COVID- 19 ulimwenguni na mkurupuko wa upele kwenye kambi ulielekeza pendekezo la mkutano wa kuratibisha washiriki kuanzisha Kikundi cha Utendaji kazi wa Usafi katika Jamii katika eneo la Kiziba, kikisaidiwa katika uenyekiti na kikundi cha Ulinzi wa Jamii katika eneo la Karongi huku kikipata msaada kutoka kwa washiriki wa afya na wa mpango wa WASH.

Malengo hususan ya desturi hii yalikuwa ni: kukadiria kikamilifu hali ya usafi (ufahamu, uzoefu na raslimali) katika kambi kwa kutengeneza vifaa na tafiti za kitaalam za kukagua ambazo zinawajali Watu Husika; kukarabati vifaa vya kambini vya mpango wa WASH kwa kutoa mapendekezo kulingana na matokeo ya ukaguzi na utafiti; kuwawezesha wanaohamasisha jamii kuwajibika na kuwapa uwezo wa kufuatilia usafi katika jamii na kukuza tabia za usaf; na kuhamasisha jamii ya wakimbizi kuchukua hatua za kujikinga COVID- 19 kwa kuwaalika wanajamii (hasa vijana wao) kuwa mstari wa mbele katika vipindi vya kuhamasisha jamii na shughuli zingine za utathmini.

Vipengele vikuu vya mkakati ni vifuatavyo:

- Ukaguzi wa Usafi wa Kijamii: Fomu za ukaguzi wa usafi zilitengenezwa kwa pamoja na kikundi cha utendakazi na timu hii ikawezesha ukaguzi wa usafi katika manyumba, shule na miradi ya ulishaji wa wanafunzi shuleni. Mapendekezo kulingana na matokeo ya ukaguzi yaliwasilishwa kwa washiriki wa mpango wa WASH ili kuboresha vifaa vya mpango wa WASH kabla ya kuanza kwa mlipuko wa COVID- 19 nchini Rwanda.

- Mkakati wa Mfanyakazi wa Afya na Usafi katika Jamii: Mkakati huu ulianzishwa katika mji wa Kiziba ili kutengeneza makundi yaliyo na mhudumu mmoja wa afya ya jamii na wafanyakazi kadhaa wa jamii wa mpango wa WASH kwa kusudi la kutekeleza matembezi ya nyumbani na kampeni za uhamasisho.

- Kuhamasisha jamii na jitihada za pamoja za jamii:Timu ya Ulinzi wa Jamii iliwaalika kikundi cha vijana wanaofoka na wanaoimba (kinachoitwa'DJ MC Orphans'), ili kuwezesha kampeni ya uhamasisho wa jinsi ya kujikinga COVID19.

- Kuwaweka wakimbizi kuwa lengo katika utafiti wa COVID- 19 katika kambi ya wakimbizi: Kwa ushirikiano na Chuo kikuu cha Global Health Equity, na kupata kibali kutoka kwa Wizara ya Elimu na Wizara ya Udhibiti wa Dharura (MINEMA), vijana wakimbizi katika Kiziba walifanya utafiti kupitia njia ya simu kuhusu Ufahamu, Mitazamo na Mazoea (KAP) ya COVID- 19 - utafiti wa kwanza wa aina hii kufanyika katika kambi ya wakimbizi uliotekelezwa na vijana wakimbizi. Vijana wakimbizi walihusika vilivyo katika kubuni na kutekeleza mradi, hivyo kukuza maarifa na uzoefu wao katika utafiti. 


\title{
Matokeo
}

- Ikiwa kama hatua ya pamoja ya kuzuia COVID- 19, jamii ya wakimbizi imejenga sehemu 122 zaidi za uoshaji mikono ili kukuza usafi kambini.

- Matokeo kutoka kwa utafiti kuhusu KAP uliashiria kuwa ufahamu kuhusu COVID- 19 kambini ulikuwa wa kiasi cha juu na kuwa wengi wa wakimbizi katika eneo la Kiziba walitambua mawasiliano ya afya katika jamii kuwa asili njema ya habari. Isitoshe, wakimbizi katika eneo la Kiziba walipata alama ya juu katika KAP inayohusiana na uoshaji mikono (ikilinganishwa na mazoea mengine ya usafi) hivyo kuakisi kwa kiasi athari chanya ya jitihada za pamoja za jamii katika kuanzisha maeneo ya uoshaji mikono.

- Wanawake walipata alama a chini kuliko wanaume kuhusu ufahamu unaousiana na COVID- 19 kutokana na wao kutojua kusoma na kuandika na kutoweza kufikilia simu/redio.

\author{
Washiriki: \\ Shirika la Africa Humanitarian Action \\ Shirika la Alight
}

Kwa habari zaidi, wasiliana na:

Ruizhi Zhu: zhur@unhcr.org; rwaki@unhcr.org

Speciose Buwamaria: buwamari@unhcr.org

\section{Mfano wa Kisa kutoka nchini Uganda: Uhamasisho wa Jamii kupitia Baraza la Maslahi ya Wakimbizi katika Kuzuia na Kuitikia COVID- 19}

M abaraza ya Maslahi ya Wakimbizi (RWCs) yalianzishwa katika makazi ya wakimbizi ya Imvepi katika mwaka wa 2017 ili kuongeza uhamasisho wa jamii na uratibu katika idadi jumla ya wakimbizi. Ikiwajumuisha viongozi wa jamii ya wakimbizi, jukumu lao kuu ni kutambua, kuzuia na kuitikia hatari katika mazingira yao. Hii inajumuisha kuweka mifumo ya mikakati chanya ya kujilinda; utetezi katika eneo; kukuza ufahamu kuhusu haki za kibinadamu, sheria na matibabu, huduma za kisheria na kisaikolojia; na kukuza ushiriki wa makundi tofauti ya AGD katika maamuzi yanayohusiana na ulinzi.

Kutokana na juhudi za RWCS washiriki wanaoshughulikia ulinzi na serikali (OPM) na UNHCR waliweza kupendezwa na kukubali kuitikia masuala na mahitaji ya ulinzi, huku Watu Husika wakiweza kupata habari zaidi na wakaweza kufikilia huduma mwafaka za ulinzi katika makazi yao. Kutokana na mlipuko wa COVID- 19, RWCs yanawezeshwa ikiwa ni njia ya kukinga na kuitikia COVID- 19 katika maeneo yafuatayo:

- Kupunguza hatari au kukatiza matumizi ya mikakati hasi ya ulinzi

- Kushinikiza mikakati chanya iliyopo (hasa miongozo ya COVID- 19 ya Shirika la Afya Ulimwenguni na Wizara ya Afya)

- Kuunga mkono mbinu mpya na chanya za kukinga na kuitikia zisizo sambamba na baadhi ya mazoea ya kipindi kabla ya COVID- 19 (kama vile kukaa nyumbani, uoshaji mikono wa mara kwa mara kwa kutumia sabuni, kukaa mbali na mwenzako, kuvaa barakoa).

Mabaraza ya Maslahi ya Wakimbizi ndio watu wa kwanza/mgusano wa kwanza katika makazi ambao hutoa habari kwa washiriki wote kuhusiana na Watu Husika. Kutoka kwa RWCs, waohupata mwongozo kuhusu utekelezaji wa shughuli katika vipindi vya kusitishwa kwa shughuli za kawaida, na jinsi ya kutambua, kukadiria na kusaidia wale walio hatarini zaidi. Hivyo, RWCs hufanya kazi ya pamoja na washiriki wa utekelezaji na uendeshaji, UNHCR, mashirika mengine ya Umoja wa Mataifa (UN) (Shirika la Chakula Ulimwenguni (WFP), Hazina ya Dharura ya Watoto ya Umoja wa Mataifa (UNICEF), Hazina ya Umoja wa Mataifa kuhusu Idadi ya Watu (UNFPA), Wanawake wa Umoja wa Mataifa, na kadhalika) na OPM kila siku ili kuhakikisha kuwa huduma bora za ulinzi (ulinzi kwa watoto, SGBV, ulinzi wa jumla, ulinzi wa kisheria na kimwili, ulinzi wa kijamii unafikia Watu wote Husika.

Huku washiriki wengi wakifanya kazi kwa njia ya simu wakati wa COVID- 19, uratibu kati ya washiriki na jamii umeweza kuboreshwa na RWCs yanayotoa ripoti za kila siku kuhusu hali ilivyo kwa UNHCR na kufuatiliza hatua zinazopaswa kuchukuliwa wanazopewa na washiriki hawa. RWCs pia yamesaidia kuwezesha mawasiliano bora na jamii za wakimbizi katika kupunguza kupingwa kwa kanuni mpya zilizoletwa na COVID- 19 (kama vile miongozo mipya ya kusambaza chakula na miongozo ya kitaifa ya kujikinga COVID- 19).

Isitoshe, jukumu la uongozi la RWCs katika shughuli za jamii, limesababisha athari chanya katika jamii. Kwa mfano, RWCs 
yamewezesha mtazamo mpya wa kazi ya jamii ambapo malipo hayalazimishwi baada ya kufanya kazi ile. Jamii pia hujuika katika kufanya kazi iliyotengewa wafanyakazi wanaopewa marupurupu hivyo kuharakisha juhudi zozote za usambazaji na kupunguza mikusanyiko mikubwa ya watu. Hii inaonekana wazi wakati wa kusambazachakula, bidhaa za usafi wakati wa hedhi na matukio ya jamii kama vile mazishi.

RWCs pia yanatumika katika kuwasiliana na jamii ya eneo husika, kusuluhisha mizozo inayohusiana na ardhi, wanyama waliopotea, kuni na fito za ujenzi wa makazi kwa njia ya uhuru. Wao huhusisha jamii ya eneo husika kwa maongezi ya kuishi pamoja kwa amani, na kuhusisha OPM na UNHCR kama mashahidi ili kukubali hatua zinazopaswa kuchukuliwa walizokubaliana.

Ujenzi wa makazi kwa Watu wenye Mahitaji Maalum katika eneo wakati wa mlipuko umetekelezwa na RWCs ambao waliwahusisha jamii ya wakimbizi katika shughuli hii. Kwa mfano, katika Eneo la 2 (Kijiji cha 14), chini ya uongozi wa Mwanabaraza wa kike wa Masuala ya Wakimbizi, vijana walihamasishwa kurekebisha makazi matano ya Watu wenye Mahitaji Maalum wakati wa mlipuko huu.

\section{Washiriki:}

Baraza la Wakimbizi nchini Denmark

Ofisi ya Waziri Mkuu

Mshiriki wa UNHCR anayetekeleza shughuli

Kwa habari zaidi, wasiliana na:

Willian Ngeze: ngeze@unhcr.org

Faith Kamoyo: kamoyo@unhcr.org

\section{Desturi Zinazojitokeza kutoka Uganda: Miungano ya Utunzi wa Jamii}

I atu wenye Mahitaji Maalum kama vile Watu wanaoishi na Ulemavu, wale wenye hali mbaya ya kimatibabu, wanawake walio hatarini, wazee na watoto wasiokuwa na watu wanaoandamana nao katika makazi ya wakimbizi huwa na changamoto zinazohusiana na kuweza kufikilia huduma pamoja na msaada wa chakula, afya, elimu, maji na makao. Pia, mara nyingi wao huwa hatarini ya kupata COVID- 19 kali sana. Kwa vile kulikuwa na idadi ndogo ya wafanyakazi wa ulinzi kwa jamii katika maeneo kutokana na COVID- 19, kulitokea pengo katika kutambua, kukadiria na kutoa rufaa kwa Watu wenye Mahitaji Maalum hadi kwa watoa huduma wanaohusika. Mtazamo wa Miungano ya Utunzi kwa Jamii ulioelezwa hapa chini unalenga kuboresha kutoa huduma na kuwasaidia Watu wenye Mahitaji Maalum.

Uhaba wa raslimali zilizotengwa ili kuwalinda Watu wenye Mahitaji Maalum katika utendakazi katika Adjumani ulielekeza kuwepo kwa hitaji la mtazamo unaolenga jamii katika kushughulikia mahitaji ya idadi hii ya watu. Kupitia Makubaliano ya Ushirikiano katika Mradi na Shirika la Lutheran World Federation, mtazamo wa Muungano wa Utunzi wa Jamii ulianzishwa. Mtazamo huu unatekelezwa kupitia muundo wa kijamii unaoitwa Timu za Miungano za Utunzi wa Jamii (CCCTs). Timu hizi zinatengenezwa kufuatia mashauriano na viongozi wa Mabaraza ya Maslahi ya Wakimbizi (viongozi wa wakimbizi katika makazi tofauti). Vizuizi vya kitaifa vya COVID- 19 vimejumuisha kukatizwa kwa usafiri (isipokuwa ule wa huduma muhimu), kufungwa kwa mipaka, kukaa mbali na mwenzako, kusitishwa kwa makusanyiko ya umma na kupunguzwa kwa idadi ya watu katika maeneo ya kazini hadi kufikia asilimia 30. Katika hali hizi, CCCTs zimetumika katika kuwasaidia Watu wenye Mahitaji Maalum na katika kuhakikisha kuwa watu hawa wanaweza kufikilia huduma na msaada bila kuzingatia idadi ndogo ya wafanyakazi mashinani.

\section{Vipengele Vikuu:}

Vipengele vikuu vya mtazamo huu vinajumuisha yafuatayo:

- $\quad$ Uramanishaji Taasisi wa Kimsingi

- $\quad$ Kutoa mafunzo kwa Wafanyakazi wa Ulinzi katika Jamii kuhusiana na mkakati huu (kusaidia katika kutekeleza mkakati)

- Mikutano ya washikadau katika jamii

- Kutengenezwa kwa CCCT

- Kupanga hatua

- Kutoa mafunzo kwa CCCT 
- Kuwatambua Watu wenye Mahitaji Maalum katika makazi na kuwaunganisha na muundo huu

- Kutoa mafunzo kwa watunzi wa Watu wenye Mahitaji Maalum

- Kutoa usaidizi kwa CCCT

- Ufuatiliaji na utoaji ripoti

- Kuhamasisha raslimali ili kusaidia utekelezaji wa shughuli za jamii na CCCTs

Kufikia umbali huu, jumla ya wakimbizi 129 (66 wa kike na 63 wa kiume) ni wanachama wa CCCT.. Wanachama wa CCCT wanapewa aproni zinazoakisi mwanga ili kuweza kutambulika kwa haraka katika jamii. Watu kadhaa wenye Mahitaji Maalum wametambuliwa na kutathminiwa.Kuna ufahamu ulioongezeka kuhusu huduma ya jamii na maeneo ya msaada kwa Watu wenye Mahitaji Maalum kupitia mafunzo ambayo yamefanyika na njia za kutoa rufaa za huduma/ msaada kama huu ukaboreshwa kwa kuhusisha CCCTs ili kuwaongoza Watu wenye Mahitaji Maalum katika makazi. Kuhusishwa kwa viongozi wa wakimbizi katika mikutano ya kuanzishwa na ya kutoa majibu baada ya kila robo umesababisha mkakati huu kuratibiwa vyema. Mwishowe, uwezo wa jamii kuitikia Watu wenye Mahitaji Maalum katika hali zenye uhaba wa huduma umekuwa dhabiti.

\section{Washiriki:}

Shirika la Lutheran World Federation

Kwa habari zaidi, wasiliana na:

Elvira Ndanga: ndangael@unhcr.org

Elizabeth Kaboyo: Elizabeth.kaboyo@lutheranworld.org

Ajavu Patrick: patrick.ajavu@lutheranworld.org

Deogratias Acidri: acidri@unhcr.org

Prosscovia Achiro: achiro@unhcr.org

\section{Desturi Zinazojitokeza kutoka Uganda: Msaada wa Makao kwa Watu wenye Mahitaji Maalum}

\footnotetext{
ufungwa kwa muda kwa kituo cha mapokezi cha Kabazaana kutokana na COVID- 19 kulisababisha uhamisho wa dharura wa Watu Husika waliokuwa wakiishi katika kituo kile. (Hili lilifanyika kwa kuzingatia mahitaji ya kukaa mbali na mwenzako na kuepuka msongamano wa watu). Hivyo basi, watu waliokuwa hatarini na hasa wale ambao hawakuwa wamejengewa makao kufikia wakati huo awakuwa na mahali pa kulala. Watu hawa pia hawakuwa na uwezo wa kujitengenezea makazi wao wenyewe. Hali hii iliwaacha Watu wenye Mahitaji Maalum kuwa hatarini ya kukosa ulinzi sawia na hatari za afya zinazoletwa na hali ya anga yenye ubaridi na mvua.
}

Ikiwa kama suluhisho la tatizo hili, jumla ya majengo 74 ya muda yamejengwa, ambayo yametumika kuwapa makao watu 65 walio hatarini na kina mama 9 wasiokuwa na waume. Mtazamo wa kijamii ulitumika katika kujenga makao haya ikijumuisha kutoa kwa vifaa vya kuweka paa (paa za plastiki zilizotolewa na UNHCR kama sehemu ya kifurushi cha Bidhaa za Kimsingi za Msaada) kwa hasa watu walio hatarini. Viongozi wa jamii na wafanyakazi wa jamii walijukumiwa kukadiria, kusimamia na kufuatilia ujenzi huku wanajamii wenye uwezo walifanya ujenzi huu kwa gharama iliyopunguzwa pamoja na kuhakikisha usalama wa mali ya hasa watu walio hatarini. Shirika la ALIGHT lilichangia dola 53 kwa kila makao katika ununuzi wa vifaa kama vile fito, matete, misumari, usafirishaji na ujenzi.

\section{Matokeo}

Mkakati huu umekuwa na matokeo mema kutoka kwa mtazamo wa Watu wenye Mahitaji Maalum na wanajamii wengine. Alipoulizwa maoni yake kuhusu msaada wa makao, mmoja wa waliofaidika alijibu, 'Nimefurahi sana kuwa nyumba yangu, mahali ambapo nina faragha yangu binafsi. Hata ikiwa watatueleza turudi kwenye kituo cha mapokezi baada ya mlipuko huu, sipendelei kurudi huko. Niko tayari kuendelea kuishi hapa. Shukrani kwa shirika la Alight.' Vivyo hivyo, mfanyakazi wa jamii alisema, 'Ninathamini mtazamo ambao shirika la Aligt linatumia ambapo wanajamii wanasaidia wenzao katika kutengeneza manyumba haya na hasa watu walio hatarini na jambo hili litawasaidia kuweza kuzoea mahali pale kwa haraka. Mimi kama mfanya kazi wa jamii, ninafurahi kuona kuwa wanajamii wangu wanapata msaada kutoka kwa shirika la Alight katika kipindi hiki ambapo mlipuko huu umetuathiri sisi wote.'

\section{Washiriki:}

Shirika la Alight

Kwa habari zaidi, wasiliana na:

Davis Mugumya: mugumya@unhcr.org

Sylvia Samanya: samanya@unhcr.org 


\title{
Mfano wa Kisa kutoka nchi ya Sudan Kusini: Jopokazi ya COVID- 19 Inayoongozwa na Jamii
}

\begin{abstract}
li kuzuia kuenea kwa COVID- 19 katika kambi za wakimbizi za Ajoung Thok na Pamir, huduma zilizohusisha watu kukusanyika pamoja na mgusano wa moja kwa moja zilipunguzwa. Ofisi za UNHCR zilisitisha shughuli za kuenda mashinani na kushirikisha jamii moja kwa moja. Hili ililisababisha pengo katika usambazaji wa kawaida wa habari kwa jamii ambao huwa wa haraka na bora kuhusiana na hatua za kujilinda na habari jumla kuhusiana na COVID- 19. Uandikishaji wa wakimbizi pia uliathirika huku shughuli husika zikifanyika tu mbali na maeneo husika. Huku ukuzaji wa ufahamu ukifanyika kupitia redio, utathmini wa maoni kutoka kambini ulionyesha kuwa wakimbizi wengi bado walikuwa na maswali.
\end{abstract}

Kwa muhtasari, kulikuwa na hitaji la mawasiliano mwafaka kuhusiana na shughuli za jamii kwa kufuata miongozo ya COVID -19; utambuzi wa haraka na kutoa rufaa kwa Watu wenye Mahitaji Maalum na watoto walio hatarini; na kampeni zenye habari kuhusiana na mbinu mpya ya usajili inayofanyika mbali na maeneo husika. Hivyo, mkakati ulitengenezwa ili kukuza ufahamu kuhusu COVID- 19; kufuatilia uvumi kuhusu COVID- 19 katika jamii; kutambua na kuwapa rufaa Watu wenye Mahitaji Maalum ili wapate huduma wanazohitaji wakati wa mlipuko huu.

Kwa haswa, jopokazi za COVID- 19 zilianzishwa na viongozi wa baraza ya wakimbizi katika kambi mbili zikiwa na malengo ya kusaidia UNHCR na washiriki wake kukuza mawasiliano ya pande mbili na jamii ya wakimbizi, na kutoa habari ya mahitaji (hasa ya Watu wenye Mahitaji Maalum wakati wa COVID- 19), na hatua za tahadhari zinazotekelezwa. Katika kambi ya Ajuonga Thok, jopokazi ya COVID- 19 Inayoongozwa na Jamii iliwahusisha wanachama 16 (4 wa kike na 13 wa kiume). Katika kambi ya Pamir, jopokazi hili liliwahusisha wanachama 10 (5 wa kike na 5 wa kiume). Jopokazi hizi zote zilisimamiwa na wenyekiti wa kambi.

Jopokazi za COVID-19 zinazoongozwa na jamii pia zinasaidia UNHCR katika usajili wa mashinani kwa kunakili habari binafsi za watoto wazaliwa katika jamii zao.Wao hufanya kazi na washiriki wa huduma ya afya katika kutambua wakimbizi wanaosafiri kurejea kwenye kambi hizi mbili ili kufuatilizwa na washiriki wa huduma ya usafi na mapokezi ya habari inayohusu COVID19 ya mara moja. Pia wanaripoti kuhusu kadi za mgawo ambazo azitumiki, na zile zilizopotea na zinahitaji kubadilishwa na kuamilishwa tena ili kupokea ulinzi kutoka kwa UNHCR kwa niaba ya wakimbizi. Jopokazi hizi zinashiriki katika mikutano ya kila juma ya Baraza la Wakimbizi ambayo pia huhudhuriwa na UNHCR, Baraza la Wakimbizi nchini Denmark na shirika la LWF linahusika na ulinzi kwa watoto. Jopokazi katika kambi zote mbili zinasaidiwa vipaza sauti, barakoa, vinywaji na vifaa vya kuandika na Baraza la Wakimbizi nchini Denmark ili kuweza kutekeleza vipindi vya kukuza ufahamu katika jamii kwa ubora.

Ushirikiano:

Baraza la Wakimbizi nchini Denmark

Shirika la Lutheran World Federation

Kwa habari zaidi, wasiliana na:

Ivy Wahome: wahomei@unhcr.org

Regina Khamis Wude: wude@unhcr.org

Abraham Mijok Ayuel Dau: dau@unhcr.org

\section{Mfano wa Kisa kutoka nchi ya Sudan Kusini: Utengenezaji wa Barakoa}

fisi ya mashinani ya UNHCR katika eneo la Malakal imeanzisha utengenezaji wa barakoa na wakimbizi wa ndani na jamii inayoishi katika eneo ili kama njia ya kupata mapato na shughuli ya kukuza amani ili kuhimili athari za COVID- 19. Ofisi ndogo ya Jamjang inaangazia uwezekano wa kuhusisha wanachuo waliohitimu kutoka kwa vituo vya ukufunzi vya kiufundi (kutoka kwa wakimbizi na jamii inayoishi katika eneo lile) ili kutengeneza barakoa zinazoweza kutumika mara nyingi pindi bajeti iliyopo itakapothibitishwa.

Washiriki: haihusiki

Kwa habari zaidi, wasiliana na:

ViTran: tran@unhcr.org 


\section{Mfano wa Kisa kutoka nchi ya Sudan Kusini: Kituo cha Redio cha Jamii}

$K^{3}$ atika kambi za wakimbizi za Jamjang, UNHCRimefanya kazi na kituo cha redio cha Jamjang FM ili kueneza habari kuhusiana na kujikinga COVID- 19. Mbinu tofauti za utumaji wa jumbe zimetumika ikijumuisha matangazo na vipindi vya maongezi kwenye redio. Isitoshe, baadhi ya wakimbizi wanaendelea kutengeneza maigizo mafupi yatakayopeperushwa kwenye kituo cha FM cha redio yanayohusu jumbe muhimu za kujikinga na kuitikia COVID- 19. Katika baadhi ya vipindi, UNHCR na madaktari kutoka kwa washiriki wanaoendeleza shughuli za kimatibabu wamehusishwa ili kusaidia katika kutibua dhana zilizoko. Mipango inaendelea ya kupeperusha kipindi ambacho kitakuwa cha kujibu maswali, uvumi na kuzungumzia baadhi ya dhana potofu katika jamii kuhusiana na COVID- 19.

Kuna itaji la kuakikisha kuwa Watu wengi Wanaohusika wanaweza kufikilia mitambo ya redio ili kuwasaidia kufikia jumbe hizi. Mitambo inayopendekezwa ni redio za simu za rununu kwani zinaweza kutumika kama simu za kuwasiliana na jamii na kutoa maoni pamoja na kutumika kama redio ya kupata habari muhimu kuhusu afya ya umma inayoenezwa na Wizara ya Afya nchini Sudan Kusini sawia na UNHCR na washiriki wake kupitia Jamjang FM.

Jitihada za kuhakikisha ubadilishanaji wa mawasiliano kati wa Watu Husika, UNHCR na wanaotoa huduma ya afya kwa jamii katika kueneza habari kuhusu COVID- 19 kupitia vituo vya redio zinaendelea. Tathmini za maoni ya jamii za dharura zimefanywa kimsingi kupitia wafanyakazi wa afya kuwauliza wanajamii wanaotembelea vituo vya afya. Jamii inapendezwa na jumbe zinazotumwa na inapokea habari sahihi inayopeperushwa kupitia redio.

Washiriki:

Jamjang FM

Kwa habari zaidi, wasiliana na:

Tamar Joanian: joanian@unhcr.org

\section{Desturi Zinazojitokeza kutoka Sudan Kusini: Uratibu wa Kibinadamu wa Kuitikia COVID- 19}

\footnotetext{
K ikundi cha Utendakazi cha Mawasiliano kuhusu Hatari na Kushirikisha Jamii (RCCE) cha Maban kimetajwa katika matukio kadhaa na Kikundi cha Kitaifa cha Utendakazi wa Kiufundi cha RCCE nchini Sudan Kusini kama mfano wa 'desturi bora zaidi ua ushirikiano wa mashirika tofautitofauti katika eneo.' Hakuna mbinu ya uratibu ya RCCE katika mwitikio wa mlipuko wa COVID- 19 sawia na hii imetekelezwa na washiriki wa huduma za kibinadamu mahali pengine nchini Sudan Kusini. Kufuatia kuanza kwa COVID- 19 nchini Sudan Kusini, washiriki wanaotoa huduma za kibinadamu katika eneo la Maban waliamilisha Kikundi cha Utendakazi cha RCCE cha Maban wakiwa na malengo ya kupatanisha mtazamo wao kwa mikakati ya mawasiliano kuhusu hatari ya COVID- 19. Kikundi cha Utendakazi cha RCCE kinaongozwa na serikali (Idara ya Afya ya Kaunti ya Maban) na kuongozwa kwa ushirikiano na UNHCR. Jumla ya washiriki 21 wa huduma za kibinadamu wameunga mkono kuwa wanachama wa Kikundi cha Utendakazi cha RCCE cha Maban kwa hiari yao ambacho hufanya mikutano yao mara mbili kwa kila juma.
}

Kikundi cha Utendakazi cha RCCE cha Maban kina makundi manne madogo: 1) Kamati ndogo ya Kufuatiliza na Kudhibiti Uvumi kuhusu COVID- 19, 2) Kamati ya Utendaji kuhusu Tabia za COVID- 19, 3) Kitengo cha Ufuatiliaji, Utathmini, Uwajibikaji, Utafiti na Mafunzo, na 4) Kikundi Kidogo cha Utendakazi cha kutoa Msaada wa afya ya Kiakili na Kiasikolojia. Majukumu ya kila kikundi hiki yanaelezwa hapa chini.

\section{Kamati ndogo ya Kufuatiliza na Kudhibiti Uvumi kuhusu COVID- 19: Kinaongozwa na Shirika la Internews na kushirikisha Baraza la Wakimbizi nchini Denmark.}

- Kutoa mafunzo kwa wakufunzi kwa washiriki wote wanaotoa huduma ya kibinadamu katika Maban kuhusu ufuatiliaji bora na udhibiti wa uvumi, habari potovu na masuali.

- Kuwasilisha mafunzo kwa mashirika yanayotoa huduma katika eneo.

- Kutengeneza zana ya mtandao ya kufuatiliza inayoshirikisha mashirika kadhaa ambayo washiriki wote katika eneo la Maban watatumia kuripoti kuhusu uvumi.

- KutengenezaRipoti ya Kudhibiti Uvumi ya mara kwa mara ambayo inanakili uvumi unaoenezwa katika jamii pamoja na majibu yaliyopangwa. Ripoti hii huelezwa Kamati Ndogo ya Kitaifa ya Kufuatilia na Kudhibiti Uvumi.

- Kutengeneza njia ya kudhibiti uvumi unaohakikisha maoni kuhusiana na Ripoti ya Kudhibiti Uvumi unafikia jamii. 


\title{
Kamati ya Utendaji kuhusu Tabia za COVID- 19: Inaongozwa na Shirika la Save the Children International na kushirikisha shirika la Relief International katika uongozi.
}

- Kutoa ushauri kulingana na ushahidi kwa mamlaka ya eneo husika na washiriki kuhusu mikakati ya kubadilika kwa tabia kwa muda mfupi na kwa muda mrefu ili kuhakikisha wakimbizi wanachukua mienendo ya kujikinga COVID19.

- Kuongoza kampeni mbili za uhamasisho wa COVID- 19 na miradi miwili ya utafiti, ikijumuisha msaada kutoka kwa washiriki wanaotoa huduma za kibinadamu ili kunakili hadithi za mawakala wa mabadiliko wa COVID- 19.

\section{Kitengo cha Kufuatilia, Kutathmini, Kuwajibisha, Utafiti na Mafunzo (MEARL): Kinaongozwa na Shirika la Chakula Ulimwenguni na kushirikisha shirika la Jesuit Refugee Service na UNHCR.}

Kufuatilia ukusanyaji data ya Mawasiliano kuhusu Hatari na Kushirikisha Jamii

- Washiriki wanaotoa huduma ya kibinadamu katika eneo la Maban huwasilisha data yao ya ufuatiliaji wa Mawasiliano kuhusu Hatari na Kushirikisha Jamii kwa Kitengo cha Kufuatilia, Kutathmini, Kuwajibisha, Utafiti na Mafunzo kila juma. Habari hii huunganishwa na kuwasilishwa kila juma kwa Kikundi cha Utendakazi cha Kiufundi cha Kitaifa cha RCCE. Kitengo cha MEARL pia hushirikisha ripoti za utathmini wa data ya kiuchunguzi kwa washiriki wanaotoa huduma ya kibinadamu kwa pamoja na data ya kimsingi ya RCCE.

- Kufuatia mkutano wa uhamasisho wa Kikundi cha Utendakazi cha RCCE katika eneo la Maban uliokusudiwa kukuza ushirikiano wa data kati ya washiriki wanaotoa huduma za kibinadamu, Kitengo cha MEARL kilipanga mafunzo ya mashirika mbalimbali ya sehemu kuu za kufuatilia na kutathmini (M\&E) katika Kaunti ya Maban, na kukuza uwezo wao wa kutumia zana ya kufuatilia iliyolinganishwa.

- Kitengo cha MEARL kimesaidia Kamati ya Uendeshaji wa Mienendo kutekeleza miradi miwili ya utafiti inayoshirikisha mashirika mbalimbali ya RCCE: 1) Utafiti wa haraka kuhusu Ufahamu, Mitazama na Mazoea (KAP) ambao ulifanya kwa kambi zote na jamii zinazoishi katika maeneo hayo; na 2) Utafiti kuusu jinsi bora zaidi ya kujumuisha jamii katika mikakakati ya COVID- 19. Miradi yote mbili ya utafiti iliungwa mkono na Kikundi cha Kitaifa cha Utendakazi cha Kiufundi cha RCCE na ilitengeneza muundo wa mwongozo wa sera za kitaifa za RCCE nchini Sudan Kusini.

\section{Kikundi Kidogo cha Utendakazi cha Kutoa Msaada wa Afya ya Kiakili na Kisaikolojia (MHPSS): Kinaongozwa na shirika la Jesuit Refugee Service (JRS) na kusaidiwa katika uongozi na Baraza la Wakimbizi nchini Denmark (DRC).}

- Kulainisha MHPSS katika kuitikia COVID- 19 kwa kuhakikisha mazingatio husika yanalinganishwa na kujumuishwa katika jumbe muhimu na uwasilishaji wa habari kati ya washiriki na miundo ya kijamii katika jamii zinazoishi katika eneo na jamii za wakimbizi; kuimarisha kipengele cha MHPSS katika miundo iliyoko katika jamii inayoishi katika eneo na kambi za wakimbizi; kutoa msaada na kukuza uwezo wa MHPSS, (kama vile Huduma ya Kwanza ya Kisaikolojia) kwa wafanyakazi wa mstari wa mbele, washiriki, na miundo ya kijamii; ukuzaji wa ujumuishwaji wa Watu wenye Mahitaji Maalum katika shughuli za MHPSS; kuunganisha habari kuhusiana na hali njema ya MHPSS sawia na kutoa huduma ili kusaidia utathmini wa mapengo, mwitikio ulioratibiwa na uhamasisho wa ugavi wa raslimali kwa washiriki wa MHPSS; kudumisha mawasiliano ya mara kwa mara na uhusiano na kikundi cha kitaifa cha utendakazi cha kiufundi (TWG) cha MHPSS, ikijumuisha kushiriki katika mikutano ya kitaifa ya TWG ya MHPSS; na kukuza mafunzo ya pande mbili na kubadilishana kwa mawazo na mazoea bora zaidi kati ya washiriki.

o Kikundi Kidogo cha Utendakazi wa MHPSS cha Maban kimewakilishwa katika MHPSS TWG cha Kitaifa.

\author{
Washiriki: \\ Baraza la Wakimbizi nchini Denmark \\ Shirika la Internews \\ Shirika la Jesuit Refugee Service \\ Idara ya Afya ya Kaunti ya Maban \\ Shirika la Relief International \\ Shirika la Save the Children International \\ Shirika la Chakula Ulimwenguni
}

Kwa habari zaidi, wasiliana na:

Dr. Gebrewold Petros Yohannes: petros@unhcr.org 


\section{Mfano wa Kisa kutoka nchi ya Sudan Kusini: Kushirikisha Jamii kupitia Ufasiri wa Jumbe kuhusu COVID- 19}

W

alimu wa jamii katika eneo la Kordofan wanahusishwa kusaidia kutafsiri jumbe za kujikinga COVID- 19 hadi lugha kuu za wakimbizi ili ziweze kuenezwa kupitia msaada wa viongozi katika maeneo haya. Mkakati huu unalenga kusaidia katika kukuza ufahamu na kupunguza ueneaji zaidi wa COVID- 19. Katika maeneo fulani, kama vile Khartoum, jamii zilichukua jukumu kuu kutoka awamu za kwanza za kuitikia COVID-19, kueneza mabango yenye habari na kutambua maeneo ya kuyaweka katika maeneo yao kupitia Wanaojitolea Kuwasilisha Huduma katia Jamii, viongozi wa jamii na wa kidini na Masultani.

Washiriki: haihusiki

Kwa habari zaidi, wasiliana na:

Annheli Aldhammar: aldhamma@unhcr.org

\section{Mfano wa Kisaa kutoka nchi ya Sudan Kusini: Kuhusisha Jamii kupitia Simu ya Rununu}

\footnotetext{
a atika Jimbo la White Nile na maeneo mengine, viongozi wa wakimbizi pamoja na wale wa jamii inayoishi katika maeneo haya wamechaguliwa na kupewa mafunzo kuhusiana na njia ya kutoa rufaa za COVID- 19 ili kuimarisha uwezo wao wa kuitikia visa kwa muda unaofaa na kwa njia bora. Pia wamesaidiwa kupata ada ya muda wa maongezi ya simu ili kuwezesha mawasiliano. Katika mji wa Khartoum, UNHCR inasaidia Kamati ya eneo hilo ya Afya ya Watu Husika kufanya kazi pamoja na Wizara ya Afya kufuatilia wanajamii katika kutambua dalili zinazohusiana na COVID- 19. Kitengo cha UNHCR nchini Sudan imeanzisha 'mawasiliano yenye muundo wa mti kupitia WhatsApp' hasa kwa jamii za wakimbizi kutoka Eritrea, Siria na Yemeni walioko Khartoum. Hii itasaidia uenezaji wa habari kupitia WhatsApp kati ya mtandao wa makundi ya wakimbizi na kusaidia kueneza habari kwa wakati unaofaa na kupata maoni kutoka kwa jamii.
}

Washiriki: haihusiki

Kwa habari zaidi, wasiliana na:

Annheli Aldhammar: aldhamma@unhcr.org 


\section{DHULMA ZA KIMAPENZI NA KIJINSIA/ KUPUNGUZA HATARI/ KUITIKIA}

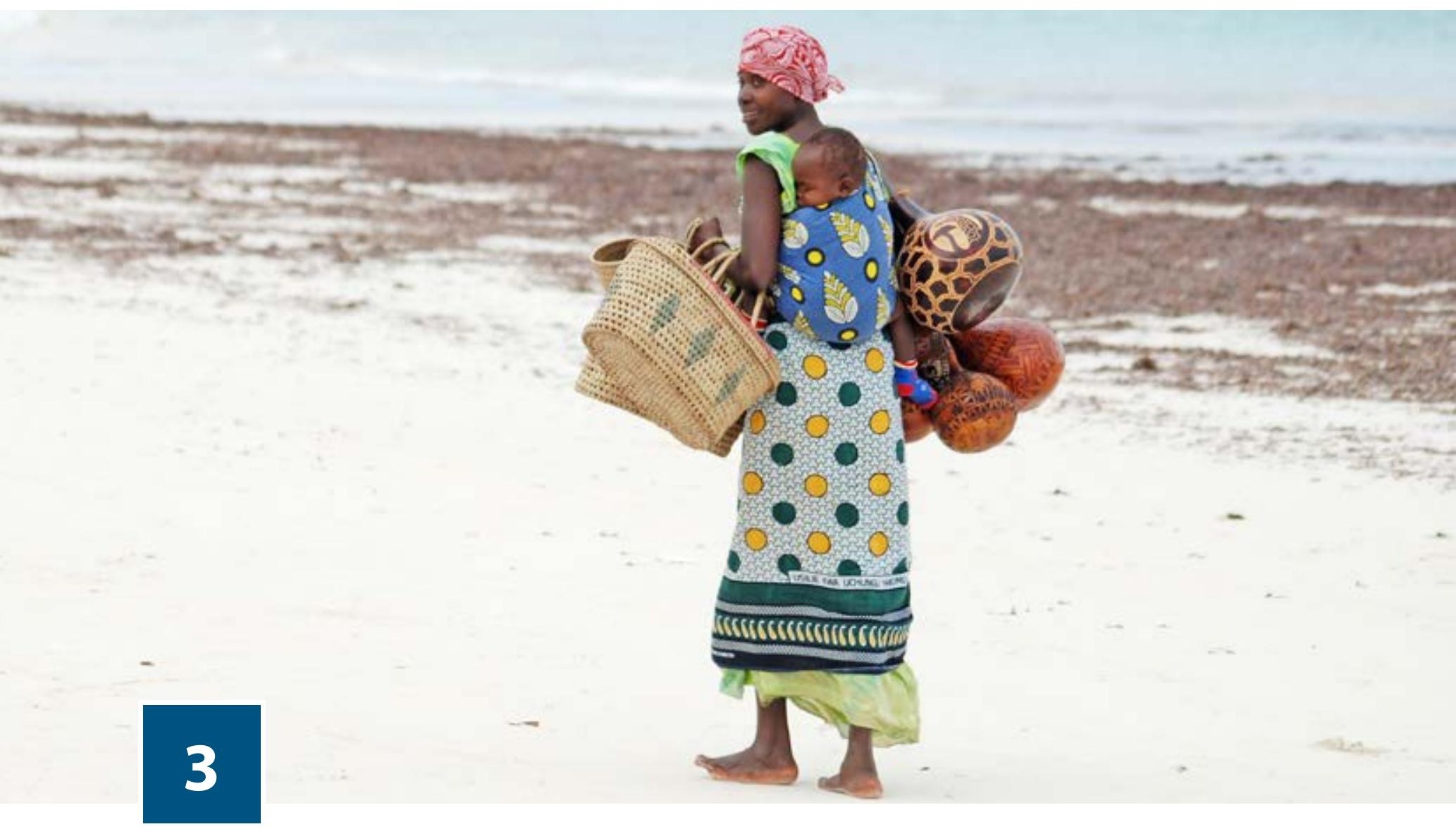

\section{Mfano wa Kisa kutoka nchi ya Burundi: Rufaa zinazotolewa kupitia Simu na Udhibiti wa Visa vya Dhulma za Kimapenzi na Kijinsia}

\footnotetext{
icha ya kusitishwa kwa usafiri nchini Burundi wakati wa mlipuko wa COVID- 19, UNHCR limeweza kuhakikisha utunzi endelevu kwa wanaonusurika kutoka kwa Dhulma za Kimapenzi na Kijinsia na uhusiano wa kuwatunza manusura wapya wanaotambuliwa kwa kutoa simu za rununu na ada ya maongezi ya kila mwezi kwa wanaotoa huduma ya ulinzi katika jamiikatika kambi za wakimbizi. Wahudumu hawa wanajumuisha viongozi wa jamii, walinda usalama, wanachama wa Kamati ya Usalama ya Pamoja kutoka kwa jamii inayoishi katika eneo, na Wenye kiti na Manaibu wao katika Kamati za Sekta mbalimbali. Mkakati huu unawezesha wahudumu hawa kuwasiliana na washiriki wanaotoa huduma husika kwa visa vya Dhulma za Kimapenzi na Kijinsia na kuwafuatilia. Pia, unawezesha UNHCR kusambaza jumbe muhimu kwa Wenye kiti na manaibu wao katika Kamati za Sekta mbalimbali ambazo husambazwa na kufikia jamii kwa ujumla.
}

Kuwezesha kutoa rufaa kwa visa vya SGBV na ufuatiliaji, wasimamizi wawili katika kila kambi walichaguliwa ili kuwasiliana na wafanyakazi wa jamii kama inavyohitajika. Visa vya dharura vya SGBV vinapewa rufaa ya huduma zinazopatikana kambini katika kipindi cha mlipuko. Pale ambapo udhibiti wa kisa unahitajika, wasimamizi hawa wanawasiliana na wafanyakazi wa jamii, mshiriki wa UNHCR anayetoa huduma ya SGBV (Shirika la International Rescue Committee) au kiini cha UNHCR kinachoshughulikia SGBV kutumia simu.

Washiriki: Shirika la International Rescue Committee

Kwa habari zaidi, wasiliana na:

Gwendolyn Roeske: roeske@unhcr.org

Brigitte Mukanga Eno: eno@unhcr.org

Christine Irambona: irambona@unhcr.org 


\section{Mfano wa Kisa kutoka Uganda: Kutoa Mafunzo kwa Miundo ya Jamii katika Kutambua, Kuripoti na Kufuatilia Masuala ya Ulinzi}

Mati wa kusitishwa kwa shughuli za kawaidia kutokana na COVID__19, visa vilivyohitaji umakinifu vilizidi kujitokeza
kutoka kwa jamii za wakimbizi, ila baadhi ya viongozi na miundo katika jamii hawakuwa na ufahamu mwafaka
kuhusiana na Ulinzi na michakato ya kuripoti inayohusiana na sekta hii.

Kwa vile miundo ya jamii na viongozi wa Mabaraza ya Maslahi ya Wakimbizi wamo katika jamii za wakimbizi, mkakati huu ulilenga kuangazia raslimali hizi zilizoko katika jamii kusaidia katika utambuzi wa visa vya ulinzi ikijumuisha matukio ya vita vya kinyumbani, na kuhusu dhulma kwa watoto na ukandamizaji. Mbali na utambuzi wa visa, kulikuwa na hitaji la visa hivi kuripotiwa hadi kwa washiriki husika ili waweze kuingilia kati.

Mkakati huu uliwezesha miundo ya jamii kwa kutoa mafunzo katika utambuzi wa masuala ya ulinzi na hatari (SGBV, ulinzi wa watoto na sheria), na kutoa ripoti kwa wakati unaofaa wa visa kama hivi ili kuweza kuingiliwa kati. Mtazamo huu umesaidia katika kuangazia mapengo katika ulinzi yaliyosababishwa na vizuizi kutokana na COVID- 19 ambavyo vilipunguza kuwepo kwa washiriki wanaotoa huduma katika jamii.

Washiriki: HADS

Kwa habari zaidi, wasiliana na:

William Ngeze: ngeze@unhcr.org

Mansur Buga: buga@unhcr.org

\section{Mfano wa Kisa kutoka nchi ya Sudan Kusini: Kutoa Rufaa kwa njia ya Simu na Vipindi vya Ushauri wa Kisaikolojia}

R hator

utoa rufaa kwa njia ya simu kunatumika ili kuwafikia wakimbizi wanaohitaji msaada, hasa katika maeneo ambayo hayafikiliwi katika majimbo ya Upper Nile, Unity, Central Equatorial na Jonglei. Katika maeneo ambayo mtandao wa simu haupatikani, washiriki wanaotoa huduma ya afya na mamlaka ya eneo huungana na kuwa kama eneo la kupewa rufaa kwa visa vya dharura. Kisha wao hutoa rufaa ya visa hivi na kuvifikisha kwa timu za Ulinzi na Uwezeshaji wa Wanawake za Shirika la International Rescue Committee (IRC) na Shirika la Lutheran World Federation (LWF) ili kuweza kusaidiwa zaidi ikijumuisha kupata ushauri wa kisaikolojia. Mashirika ya IRC na LWF yametoa mafunzo ya kimsingi kwa wafanyakazi wanaopewa marupurupu kutoka kwa jamii ya wakimbizi katika kambi za Jamjang ili kuwawezesha kutoa huduma ya kimsingi ya ushauri kwa visa vya SGBV na ulinzi wa watoto katika hali za kusitishwa kwa shughuli za kawaida ambazo zitazuia kutoka eneo moja hadi nyingine kwa wanajamii.

Washiriki:

Shirika la International Rescue Committee

Shirika la Lutheran World Federation

Kwa habari zaidi, wasiliana na:

ViTran:tran@unhcr.org 


\section{DESTURI ZISIZOHUSIANA NA COVID}

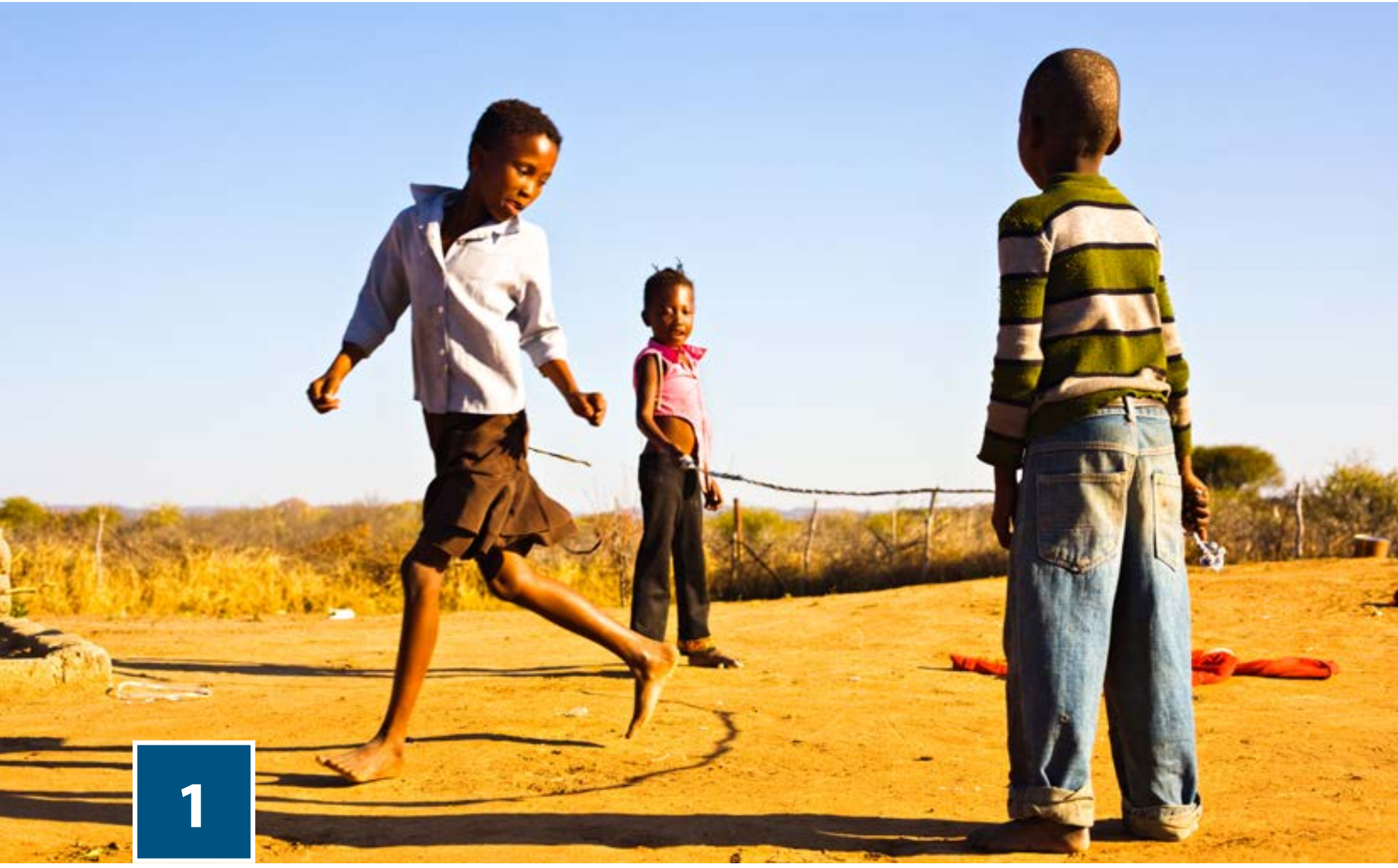

\section{KUWAKINGA WATOTO NA VIJANA}

\section{Desturi ya Kutia moyo kutoka Ethiopia: Kukadiria na Kupanga wa Elimu na Mtazamo wa Kuhusisha Jamii}

$1 \begin{aligned} & \text { kakati wa UNHCR wa utendaji wa elimu nchini Ethiopia hulenga kuboresha mafanikio ya mafunzo, kuhakikisha } \\ & \text { kuwa mashule yana mazingira salama ya kusoma, kuboresha uwezo wa kufikilia elimu ya sekondari na ya juu, } \\ & \text { kuhakikisha kuwa nafasi za masomo ni za kudumu na zinaweza kufikiliwa na watu wote kulingana na mahitaji yao, }\end{aligned}$ na kutoa nafasi za elimu upesi iwezekanavyo wakati wa hali ya dharura. Kiwango cha usajili wa mashule ni wa chini kwa sababu tofauti ikijumuisha nyakati ngumu za kiuchumi na kuhama kwa wakimbizi katika baadhi ya kambi.

Kwa kuangazia ukweli huu, mkakati huu hufanya Ukadiriaji wa Pamoja kila mwaka ili kukadiria hali jumla ya mipango ya Elimu, mapengo na mahitaji, na kuwa kama kigezo katika kupanga shughuli za mwaka unaofuata. Ukadiriaji wa Pamoja unafanyika baada ya kufungwa kwa mwaka wa shule kati ya mwezi wa Juni na Julai kutumia data bora na yote iliyokusanywa ili kupima kiwango cha utendaji ikilinganishwa na mpango wa mwaka huo. Hojaji na orodha za ukaguzi pia zinatumika. Washiriki katika ukadiriaji wa Pamoja wanajumuisha Shirika la Serikali la Masuala ya Wakimbizi na Wanaorudi (ARRA), Tume ya Maendele na Msaada baina ya Makanisa (DICAC), shirika la Shirika la Save the Children International (SCI), UNHCR, Ofisi ya Elimu ya Woreda (WEO) walimu, Vyama vya Wazazi na Walimu (PTAs) wawakilishi wa jamii na viongozi. Data ya sekondari hutolewa kutoka kwa ripoti za kila mwezi na za ufuatiliaji inayotolewa kwa ofisi za UNHCR na ARRA katika eneo. 
Wakati wa Ukadiriaji wa Pamoja, kuna mjadala kuhusiana na matekeo katika kiwango cha jamii, kuboresha ushirikisho wa wawakilishi wa jamii na uhakiki wa matokeo. Mtazamao hususa wa kushirikisha jamii unatumika kana sehemu ya mkakati kama inavyoelezwa vifuatavyo:

- Kushirikisha wawakilishi wa jamii katika Makadirio ya Pamoja: Viongozi wa kidini, Vyama vya Wanawake, Kamati Kuu za Wakimbizi (RCCs) na wanachama wengine wanaochaguliwa na jamii kulingana na uwezo wao kuwasilisha mtazamo wa jamii.

- Kufuatilia kuhudhuria kwa wanafunzi na walimu kila siku ya shule na PTAs. (Kuna PTA moja katika kila shule - jumla ya vyama 43, kila moja kikiwa na wakimbizi 7, sawia na Mabaraza ya Wanafunzi [wanafunzi 5-8 kwa kila Baraza la Wanafunzi katika shule za sekondari] na Viongozi wa Wanafunzi [wanafunzi 3-4 kwa kila shule ya msingi]).

- Kukuza uwezo wa PTAs. Kwa mfano, kufikia katikati ya mwaka wa 2020, PTAs 43 vimepokea mafunzo kuhusu majukumu yao katika mashule.

- Matumizi ya mbinu za kuwasilisha malalamishi katika shule na taratibu mwafaka za kufuatilia (kama vile visanduku vya kuwasilisha malalamishi, watendakazi maalum katika shule [baadhi yao wakishughulikia malalamiko kuhusu SGBV pekee].

- Kukuza ushiriki wa wazazi katika shughuli za shule zinazowajumuisha kama vile vipindi vya kusimulia hadithi na nyimbo vinavyoongozwa na wazazi vinavyofungamanishwa na urithi wa kitamaduni. Shughuli hizi hufanyika wakati wote mwakani ili kuboresha ushiriki wa wazazi.

Ukadiriaji huu wa Pamoja unatoa jukwaa la jamii kuzungumzia wasiwasi walio nao, kutoa mapendekezo kulingana na mipango iliyopo na kushiriki katika mchakato wa kupanga masuala ya elimu. Matokeo ya Ukadiriaji wa Pamoja wa kila mwaka hunakiliwa na kutumika kama zana ya uhamasisho na vile vile kuwa kumbukumbu ya ugavi wa raslimali na ukuzaji wa mapendekezo.

Ni muhimu kufahamu kuwa UNHCR imetengeneza mbinu mwafaka ya uratibu na Ofisi ya masuala ya Elimu katika Wilaya ili kulainisha kazi ya masomo ya UNHCR katika mfumo wa kitaifa wa elimu, kudumisha viwango vya kitaifa na kuboresha makubaliano. Jukumu la uratibu la UNHCR ni kuhakikisha muitikio bora wa washikadau tofauti tofauti katika kukuza mahitaji ya wakimbizi pamoja na ya jamii zinazoishi katika maeneo husika. Kikundi cha Utendakazi cha Elimu kinachojumuisha ARRA, UNHCR, Shirika la Chakula Ulimwenguni na washiriki wengine wanaotekeleza huduma (Shirika la Save the Children International, Tume ya Ukuzaji na Msaada Baina ya Makanisa), kilianzishwa na kimefungwa na Mkataba wa Makubaliano. Kikundi hiki kinajumuisha taasisi 43 za masomo (vituo 19 vya Utunzi na Elimu ya Utotoni [ECCE], shule 21 za msingi, shule 3 za sekondari na Chuo kikuu cha Elimu ya Ukufunzi).

\section{Matokeo}

Tangu kuanzishwa kwa mtazamo huu katika mwaka wa 2015, kumekuwa na uhudhurio na usaliaji bora katika kiwango cha shule. Kulikuwa na asilimia 83 ya hudhurio katika kiwango cha shule ya msingi katika mwaka wa 2019 na ongezeko la asilimia 53 ya usajili katika kiwango cha sule ya sekondari katika shule za wakimbizi na zile za wenyeji kwa jumla katika nusu ya kwanza ya mwaka wa 2020.

\section{Washiriki:}

Shirika la Masuala ya Wakimbizi na Wanaorudi

Tume ya Ukuzaji wa Msaada Baina ya Makanisa

Shirika la Save the Children International

Ofisi ya Elimu ya Woreda

Shirika la Chakula Ulimwenguni

Kwa habari zaidi, wasiliana na:

Muhammad Harfoush: harfoush@unhcr.org

Jael Shisanya: shisanya@unhcr.org 


\section{Desturi Inayojitokeza kutoka Kenya: Mtazamo wa Kifamilia na Kijamii katika Utunzi wa Nyumba Zinazoongozwa na Watoto}

$\int k$ tunzi wa undugu au ulezi haupatikani kila mara kwa watoto wanaohitaji huduma hii katika mazingira ya ukimbizi, kutokana na uanuwai wa tamaduni na mazoea katika kutoa utunzi huu. Ingawa, hali nyingoi katika mazingira ya ukimbizi husababisha hitaji la utunzi wa malezi ikijumuisha wakati watoto wanaachwa na wazazi wanaporejea katika nchi zao asilia; udhalimu wa baadhi ya watoto katika utunzi wa kindugu, hivyo kulazimisha utenganisho wa muda mfupi au wa muda mrefu; kukosekana kwa makazi mwafaka ya kuwapeleka watoto waliookolewa kutoka kwa mipangilio isio bora ya utunzi; na kuwasili kwa watoto wasiokuwa na jamaa wanaoambatana nao katika kambi za wakimbizi au ambao hawajapata utunzi wa malezi.

Mkakati wa wakati huu unalenga kutoa utunzi wa kuaminika wa muundo unaoongozwa na jamii ambao unatumika kama muundo wa msaada kwa Nyumba Zinazoongozwa na Watoto (CHHs); Kukoleza utambuaji salama wa malalamiko ya ulinzi kwa watoto wanaoishi katika CHHs na kuwaunganisha na mikakati mwafaka ya ulinzi; na kuwasaidia vijana waliokomaa katika kukuza stadi za maishani na stadi za kujikimu katika kambi za wakimbizi.

Kufikia hapa, mikutano ya kutoa maoni ilifanyika pamoja na CHHs ili kuwapa jukwaa la mazungumzo kuhusu masuala yanayowaathiri. CHHs ziliulizwa watambue watu wazima ambao wangependelea wawe washauri/wasimamizi wao ambao wangependa wawasaidie kwa njia mbalimbali. Washauri waliotambuliwa walijulishwa kuchaguliwa na mtoto husika na kuwaleta pamoja kwa vipindi vya uhamasisho kuhusu majukumu ya ulezi, na jukumu la mshauri/msimamizi chini ya mkakati huu ikijumuisha kuripoti wasiwasi kuhusu ulinzi ambayo watoto hawa wanaweza kuwa nayo.

Mikutano ya kutoa maoni ya kufuatilia na CHHs ilionyesha kuwa washauri/wasimamizi tayari walikuwa wakiwasaidia CHHs kwa njia mbalimbali ikijumuisha kuwachotea watoto maji wanapokuwa shuleni, kuwapikia na kuwapeleka hospitali wanapokuwa wagonjwa. Jambo hili la washauri/wasimamizi limependekezwa na kuhimizwa.

Watoto wanaohitaji walezi wanatambuliwa kutumia mbinu tofauti kama vile kupitia miundo ya jamii, vituo vya Furaha (vituo vya ulinzi kwa watoto baina ya mashirika yanayotoa huduma kwa watoto), wakati wa mifumo ya usambazaji chakula, kupitia ripoti zinazofikia ofisi za Baraza la Wakimbizi nchini Denmark au UNHCR kupitia msaada unaotolewa mashinani mara mbili katika juma au kupata rufaa kutoka kwa vitengo vingine. Rufaa mwafaka zinatolewa na kitengo cha ulinzi wa watoto cha UNHCR, kutumia njia zilizowekwa za kutoa rufaa baina ya mashirika katika kambi za wakimbizi. Ukadiriaji wa mtoto unafanywa kupitia zana za kukadiria zilizo sanifu na zenye kuzingatia maslahi ya watoto na mpango wa utunzi kupendekezwa kwa kujumuisha mtoto mwenyewe. Uwezekano wa utunzi mbadala kama vile wa undugu au mlezi huzingatiwa kwanza. UNHCR hukagua mipango ya utunzi na ikiwa utunzi wa undugu au mlezi haupatikani, patanisho la mpango wa kuishi ulio huru na unaosimamiwa hufikiliwa na mshiriki husika na mtoto mwenyewe. lli kuwezesha mchakato huu, mtoto yule husema majina ya watu wazima ambao wangependelea kuwa washauri/wasimamizi wao na watu hawa wazima wanahamasishwa kuwasaidia watoto kama ilivyoelezwa awali.

\section{Matokeo}

Tangu kuanzishwa kwake, jumla ya washauri/wasimamizi 50 (waume 16 na wa kike 34) wametambuliwa, kuhamasishwa na kuunganishwa na watoto.

\section{Washiriki:}

Baraza la Wakimbizi nchini Denmark

Shirika la Lutheran World Federation

Kwa habari zaidi, wasiliana na:

Amin Afridi: afridi@unhcr.org

Alexander Novikau: novikau@unhcr.org

Ina Guya: guyai@unhcr.org 


\section{Desturi Inayojitokeza kutoka Kenya: Miundo ya Kijamii ya Ulinzi wa Watoto}

W

asiwasi na hatari nyingi zimejitokeza za ulinzi wa watoto katika kambi za wakambizi za Daadab ikijumuisha kazi kwa watoto, kujihusisha na ngono ili kujikimu kimaisha, ujauzito wa mapema, utenganisho wa watoto kutoka kwa watunzi wao, kuacha shule, kuuzwa kwa watoto, hatari za kuajiriwa katika jeshi na ndoa za mapema/za kulazimishwa kwa wasichana wachanga. Hatari hizi zinahusiana na uwezo usiotosha wa kifedha na kukosa chakula, matumizi yaliyoongezeka ya dawa za kulevya kwa watu wachanga na ongezeko la idadi ya wanaotafuta hifadhi ambao hawajaandikishwa ikijumuisha wanaowasili na wale wanaorudi kambini baada ya kurudishwa nyumbani.

Ili kuangazia maswala ya ulinzi wa watoto katika kambi ya Daadab, UNHCR inafanya kazi na Shirika la Terre des Hommes na la Save the Children International ili kutekeleza mkakati unaotumia njia nyingi, kuangazia miundo ya kijamii kama vile Watu Wanaojitolea kwa Ulinzi wa Watoto (CPV), Wataalam Wanaomilikiwa na Jamii (CORPs), Idara za Msaada Zinazowajali Watoto, Kamati za Ulinzi wa Watoto (CPC), Baraza la Wazazi Walezi na Watoto, Kamati za Kijamii za Vijana Wanaobalehe (CBAC), Kamati za Maslahi ya Watoto (CWC), Kamati za Elimu kwa Watoto na Vituo vya Maslahi na Kina mama wa watoto ili kuwezesha jamii kuitikia wasiwasi uliopo na ule unaojitokeza kuhusiana na ulinzi wa watoto. Miundo hii ya kijamii hupokea mafunzo ili kusaidia katika kutambua wasiwasi wa ulinzi wa watoto na katika kuangazia visababishi na matokeo yake. Isitoshe, huwa wanatengeneza mikakati ya pamoja ya utambuzi, kujikinga na kuitikia inayohusiana na mahitaji na uwezo wa jamii binafsi ya ulinzi wa watoto.

Mikakati hii inakamilishwa na mitazamo ingine ikijumuisha kukuza ufahamu, kuingilia kati, kueneza habari, kutoa njia za kutoa rufaa, mafunzo kwa jamii, kutoa huduma za kimsingi za ushauri, kutengeneza na kutekeleza mipango ya hatua za jamii zinazoongozwa na vijana, kushughulikia visa vyenye hatari ya chini, kufuatilia visa vinavyopewa rufaa na kuunganisha jamii na watoa huduma ili kuimarisha na kukuza ulinzi wa watoto katika kiwango cha familia na jamii. Hasa:

- Shirika la TdH linatoa huduma ya kuwaweka watoto katika makao ya muda na ya kudumu chini ya walezi katika mpango wake wa wazazi walezi.

- Kikundi cha wazazi walezi ambao ni wakimbizi wanafanya kazi na SCI, wanajitolea kuwatunza na kuwalea watoto walioachwa na wasio na watu wanaoambatana nao (Kuna wazazi walezi 100 katika maeneo ya Ifo na Dagahaley na jumla ya watoto 542 wako chini ya utunzi wao).

- Miundo ya kijamii inasaidia katika kutambua watoto wanaodhulumiwa, kuripoti na kutoa njia za kutoa rufaa na kufuatiliza msaada kwa visa kama hivi.

- Miundo ya kijamii kama vile CPC, CORPs na CPVs inahusika katika michakato ya uingiliaji kati wa visa vya dhulma ya watoto vilivyo na hatari ya chini hivyo kusababisha kupungua kwa visa vyenye hatari ya chini katika jamii. Pamoja na viongozi wa jamii, washikadau wawa hawa wanahusika katika kutambua na kudhibitisha watakaonufaika na huduma za TdH kama vile msaada wa makazi, mafunzo na huduma za udhibiti wa visa.

- Kamati za Maslahi ya Watoto husaidia katika kukuza ufahamu na kuhamasisha jamii kuhusiana na kuzuia dhulma kwa watoto, na kuhusu desturi mbaya za kitamaduni kama vile ndoa za watoto, Ukeketaji wa Wasichana (FGM) na kazi kwa watoto. Pia wanasaidia SCI katika kukuza uwezo wa wahasiriwa wa dhulma ya watoto kupata haki. Wanatumia jumbe zinazoweza kufikiwa (zinazowajali watoto) katika uenezaji wa habari kwa watoto, wazazi/ watunzi na wanajamii. Isitoshe, wanatoa mafunzo kwa wanajamii kuhusu athari za muda mfupi na za muda mrefu za dhulma kwa watoto na jinsi ya kutambua watoto walio taabani.

- Wanachama wa kongamano la watoto wanafanya mikutano na washiriki ambapo malalamishi yao huzungumziwa na kujibiwa na washiriki wanaohusika. Kijumla, miundo ya jamii hutumika kama mbinu za kuripoti maoni ya wanaofaidika na washiriki.

\section{Washiriki:}

Shirika la Save the Children International

Shirika la Terre des Hommes

Kwa habari zaidi, wasiliana na:

Charity Chomba: chomba@unhcr.org

Virginia Njoroge: njorogev@unhcr.org 


\section{Desturi Inayojitokeza kutoka Kenya: Masomo kupitia Redio katika Jamii}

L

atika kambi ya wakimbizi ya Daadab, wanafunzi wengi hawawezi kufikilia vifaa vya mafunzo wakati shule zimefungwa. Masomo kupitia redio yanatoa fursa ya kuwafikia idadi kubwa ya wanafunzi - hasa wale wanaoweza kujisomea wao binafsi na wanaweza kujifunza bila kuhitaji msaada wa moja kwa moja kutoka kwa walimu na watunzi. Vipindi vya masomo kupitia redio huwakubalisha walimu kuendelea kuwasiliana na wanafunzi na kuwapa vifaa husika vya masomo. Wajumuisha mitandao ya mawasiliano kama vile WhatsApp, walimu na wanafunzi wana fursa njema ya kuwasiliana kwa karibu zaidi pale ambapo teknolojia inayohitajika inapatikana.

Vituo vya redio ya jamii ni muhimu katika mkakati huu kwani vituo vingi vya redio vya FM vinavyopeperusha masomo ya shule za kitaifa haviwafikii watu katika kambi za wakimbizi. Hivyo, vituo vya redio ya jamii vinawapa wanafuzi ambao ni wakimbizi firsa sawa ya kufikia vifaa vya masomo vinavyotolewa kwa wanafunzi wa vyuo vya kitaifa. Mkakati huu unahusisha ushirikiano kati ya kituo cha redio ya jamii (Redio ya Gargaar) ambayo kwa sasa inaweza kufika umbali wa kilomita 40 (kuwakilisha eneo lote na kambi na zaidi).

Mkakati huu unajumuisha matumizi ya ratiba ya redio ambayo huenezwa kutumia utumaji wa jumbe kwa watu wengi na kupitia viongozi wa jamii juma moja kabla ya kupeperushwa kwa kipindiu hiki cha masomo. Matangazo ya redio pia hutumika kuwajulisha wakimbizi na wanajamii wa eneo husika kuhusu kipindi cha redio kilichopangwa kupeperushwa. Wanafunzi wanahitajika kusikiliza kituo hicho cha redio kulingana na ratiba iliyotolewa awali. Familia zilizopakana zinaweza kutumia redio moja kwa kuiweka karibu na ua na kuongeza sauti ya mtambo.

Hati za redio za kila mada hutayarishwa na walimu waliochaguliwa wa masomo husika na kuchapishwa. Mwalimu mmoja anyehusika katika utayarishaji wa hati huchaguliwa ili kujitayarisha siku moja kabla ya kuwasilisha somo kupitia redio. Makundi ya WhatsApp yanayowajumuisha wanafunzi na walimu yanatumika ili kuwezesha mawasiliano ya moja kwa moja.

\section{Matokeo}

Mipango ya kupata masomo yaliyotayarishwa kutoka kwa Taasisi ya Kuendeleza Mtaala nchini Kenya inaendelea. Masomo haya ya kitaifa yatatumika badala ya hati zinazotumika za redio pindi tu zitakapopatikana huku walimu wakiendelea kudumisha mawasiliano na wanafunzi kupitia makundi ya muundo wa mti katika mtandao wa WhatsApp.

\section{Washiriki:}

Kwa habari zaidi, wasiliana na:

Clara van Praag: vanpraag@unhcr.org

Alan Mwika:mwika@unhcr.org

\section{Mfano wa Kisa kutoka Kenya: Kuhusisha Wanachama wa Klabu ya mpango wa WASH Shuleni}

M kakati wa Baraza ya Wakimbizi ya Norway (NRC's) wa mpango wa WASH unajumuisha kuhusishwa kwa watoto na vijana na mtazamo wenye pande nyingi na viwango tofautitofauti. Katika masule, Baraza la Wakimbizi la Norway hufanya kazi kwa ukaribu na wanachama wa klabu ya WASH (wanachama 20 katika kila shule), kutoa mafunzo kuhusu mbinu za usafi na usafishaji. Hivyo, wanachama wa klabu ya WASH waliopokea mafunzo huwafikia wanafunzi wenzao kama waelimishaji na kuwapatia habari na mbinu ambazo wamejifunza. NRC pia hutoa mafunzo kwa wasichana matineja katika mashule kuhusu usafi wakati wa hedhi.

Katika kiwango cha jamii, timu ya NRC ya mpango wa WASH inafanya kazi na vijana ili kuimarisha ushiriki wa mashirika ya kijamii na makundi ya kujisaidia kibinafsi katika shughuli za mpango wa WASH. Kufuatia lengo la Baraza la Wakimbizi la Norway kuangazia watoto na vijana, wafanyakazi wa baraza hili wamepewa mafunzo kuhusiana na Sera ya Kutoa Ulinzi kwa Watoto, na wafanyakazi wa kitaifa wa baraza hili hutia sahihi kudumisha sera hii kana sehemu ya maelezo ya kazi yao.

\section{Washiriki:}

Baraza ya Wakimbizi ya Norway

Kwa habari zaidi, wasiliana na:

Moe Miyahara: miyahara@unhcr.org

Oscar Nabiswa: nabiswa@unhcr.org

John Wagacha Burton: burtonj@unhcr.org 


\section{Desturi Zinazojitokeza kutoka Uganda: Mradi wa Kuunganisha Jamii}

M radi wa Kuunganisha Jamii katika makazi ya Imvepi ni mkakati unaosimamiwa na vijana ambao unashughulikia swala la vijana kutofanya kazi katika makazi huku ukisaidia wanajamii kuchaji simu zao. Asili ya umeme iliyo imara na inayopatikana wakati wote ya kuchaji simu ni changamoto kuu katika makazi haya ambayo huzuia mawasiliano na uratibu kati ya UNHCR, washiriki wake na jamii ikijumuisha Mabaraza ya Maslahi ya Wakimbizi. Mradi huu ulianza baada ya kibanda cha kuchaji simu cha jamii kinachotumia nishati ya jua kuwekwa. Mchakato huu unajumuisha kuunganisha majengo katika jamii (lengo hasa likiwa ni Idara za Ulinzi na Habari na Vituo vya Msaada), kwa kuwezesha kufikia mtandao wa WiFi na kwa kubadilisha majengo haya kuwa vituo vilivyounganika. Kukuwepo kwa muunganisho huu, majengo haya yamefanyika kuwa maeneo ambayowakimbizi wanaweza kupata habari, kuwasiliana na UNHCR na washiriki wenza kupitia nambari mpya za simu iliyoanzishwa (kama sehemu ya Mbinu ya Kutoa Maoni Rufaa na Suluhisho [FRRM] au kuwasiliana na wapendwa mashinani kupitia baadhi ya mifumo ya kutuma jumbe na kupiga simu.

Katika kuanzishwa kwa mradi, vijana walihamasisha na kushiriki katika shughuli za kukuza ufahamu kuhusu kuchaji simu katika kituo, kufikia vijiji vyote katika eneo la Zone 1 ikijumuisha maeneo ya ibada, sokoni, maeneo jumla ya usambazaji wa chakula na matukio ya michezo. Ikiwa kama sehemu ya mchakato huu, vijana waliafikiana na jamii kuhusu ada za kuchaji vifaa tofauti tofauti: Ugx.300/- kwa simu za kawaida, Ugx.400/- kwa simu za kisasa, Ugx.500/- kwa tochi, na Ugx.700/- kwa vipakatalishi. Watu wachanga pia waliwajumuisha RWCs katika vipindi vya kukuza ufahamu wa jamii ili kukubaliana kuhusu mradi huu. Mwenyekiti wa RWC II hasa amewapa vijana msaada mkubwa katika mradi huu kwa njia ya uhamasisho.

Mradi huu ulipoanzishwa ulikuwa na uwezo wa kuchaji vifaa vichache tu kwa wakati mmoja. Hata hivyo, UNHCR hivi punde imetoa msaada kwa kituo hiki wa chaja 20 za simu. Kwa wakati huu, kituo hiki kina uwezo wa kuchaji simu 12 hadi 15 na vifaa vingine (vipakatalishi, tochi na kifaa cha kuweza chaji ya ziada ya simu) kila siku.

Kuwepo kwa muunganisho huu kumeboresha kuishi kwa amani na kuleta pamoja vijana wa jamii ya wakimbizi na wanajamii wanaoishi katika eneo kucheza michezo ya ubao kwa pamoja na kuingiliana kwa njia zingine. Hii imeelekeza vijana hawa kupanga mkutano na wenzao wa jamii inayoishi katika eneo hilo utakaofanyika katika kituo hiki wa kuwajumuisha vijana hawa katika timu ya mradi wa uunganisho.

Watu wachanga pia wameanzisha kuuza ada ya muda wa maongezi ya simu kutokana na akiba ya mkakati wa kuchaji simu. Hili ni jambo linalofurahiwa na Watu wengi Husika ambao kwa sasa hawalazimiki kusafiri masafa marefu ili kuchaji simu zao. Vijana hawa pia walipendekeza kutengenezwa kwa vyoo vya pande mbili katika kituo. Hili ilifanyika hatimaye kwa mujibu wa shirika la International Rescue Committee huku watu wachanga wakichangia katika kuchimba choo hiki.

UNHCR na Ofisi ya Waziri Mkuu ina jukumu la kiujumla la mradi huu huku mshiriki anayetoa huduma ya ulinzi na RWCs wanawajibika katika kufuatilia matokeo ya mradi huu ya kila siku.

\section{Washiriki:}

Baraza la Wakimbizi nchini Denmark

Ofisi ya Waziri Mkuu

Kwa habari zaidi, wasiliana na:

William Ngeze: ngeze@unhcr.org

Faith Kamoyo: kamoyo@unhcr.org 


\section{Desturi Zinazojitokeza kutoka Uganda: Kuweza Kufikilia Elimu ya Sekondari}

$\mathrm{M}$

kakati huu ulitengenezwa kama muitikio wa maoni yaliyotolewa na jamii wakati wa tukio la hamasisho kwa jamii ('Kuja Kuja') na tukio la mbio za kilomita 5. Wanajamii waliwasilisha mapendekezo ya jinsi ya kuboresha jamii yao ikijumuisha pendekezo la kuwepo kwa shule ya jamii. Watoto wengi wakimbizi katika Rubondo (Nakivale) wanaokamilisha shule ya msingi hapo awali hawajaweza kuendelea na masomo ya shule ya sekondari kwa sababu ya kutembea masafa marefu hadi kwenye shule ya pekee ya sekondari (iliyoko umbali wa takriban kilomita 35), na hatari za ulinzi zinazohusiana na hali hii.

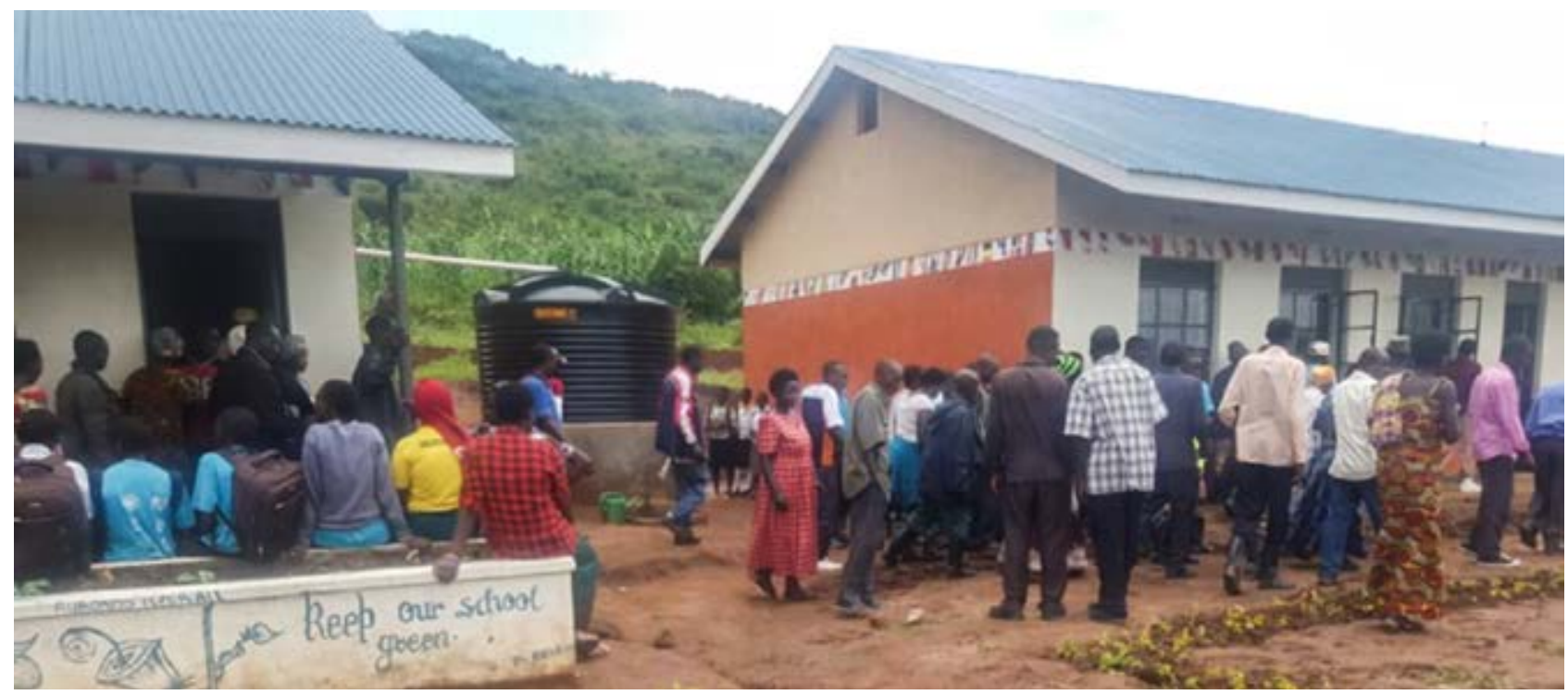

Mkakati huu unaongozwa na wanajamii ambao wanawahamasisha wazazi wote katika jamii ya wakimbizi na ya wenyeji na kuwahimiza kuwapeleka watoto wao shuleni. Wanajamii pia wanazidi kushiriki katika shughuli za kila mwezi za jamii ikijumuisha kusafisha uwanja wa shule na kuweka mipaka na kulinda mipaka ya shule kutokana na uvamizi. Wazazi hulipa karo ya shule ya Ugx 100,000 (takriban dola 27) kila muhula ili kuwezesha uendeshaji wa shule ikijumuisha malipo ya mishahara ya walimu.

\section{Matokeo}

- Usajli uliongezeka kutoka idadi ya wanafunzi 76 (32 wa jinsia ya kike) mwishoni mwa mwaka wa 2019 hadi wanafunzi 110 (52 wa jinsia ya kike) katika mwezi wa Machi mwaka wa 2020 kabla ya shule kufungwa kutokana na mlipuko wa ugonjwa wa COVID- 19.

- Usaliaji ulikuwa wa juu huku asilimia 93 ya wanafunzi waliojisajili katika mwaka wa 2019 wakisalia shuleni kufikia mwanzoni mwa mwaka wa 2020.

- Shule hii ina mpango wa kuwalisha wanafunzi, jambo ambalo huwezesha usaliaji wa wanafunzi shuleni.

- Mtazamo chanya wa shule wa jamii ni wa kutia moyo. Jamii inaunga mkono mkakati huu unaoongozwa na jamii kwa kulipa karo na kununua mahitaji mengine yanayohitajika ya kusoma na sare za shule ili watoto hawa waweze kupata elimu katika shule.

- Uratibu na ushirikiano na washiriki wengine uko imara Usimamizi wa shule unafanya kazi kwa karibu na Serikali ya Wilaya, UNHCR, Ofisi ya Waziri Mkuu, Shirika la Windle International nchini Uganda (mshiriki wa UNHCR katika kutoa huduma ya elimu) na Shirika la Alight, mshiriki wa kusaidia ukuzaji.

- Ukuzaji wa miundomisingi: Kupitia msaada kutoka kwa washikadau, hasa, Shirika la Alight, maabara ya kisayansi yamejengwa na kukamilishwa. Hili ni hitaji la Wizara ya Elimu na Michezo kwa shule zote za sekondari ili kuweza kufundisha masomo ya sayansi.

\section{Washiriki:}

Shirika la Alight

Serikali ya Wilaya

Ofisi ya Waziri Mkuu

Shirika la Windle International nchini Uganda

\section{Kwa habari zaidi, wasiliana na:}

Davis Mugumya: mugumya@unhcr.org

Sylvia Samanya: samanya@unhcr.org 


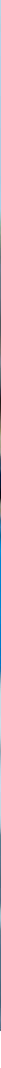

\section{ULINZI WA KIJAMII}

\section{Desturi Inayojitokeza kutoka Djibouti: Mchango wa Jamii katika Kutafuta Visa vya Kifua Kikuu kati ya Wakimbizi}

$\Gamma^{k}$

hughuli hii inalenga kuboresha utafutaji wa visa vya Kifua Kikuu (TB) katika mazingira ya jamii ya wakimbizi na kukuza uanzishaji wa mapema wa matibabu kupitia shughuli zinazojumuisha jamii. Pia hutafuta kuboresha msaada

kwa wagonjwa wa Kifua Kikuu kwa kuwafikishia utunzi karibu zaidi. Mkakati huu una vipengele sita vikuu, navyo ni, uundaji wa kamati za afya, kukuza uwezo kwa wahudumu wa afya ya jamii (CHWs) na wahudumu wa afya(HCWs), mikutano ya utathmini ya baada ya miezi mitatu, mikutano ya uhamasisho, msaada ya kilishe na kufikisha matibabu katika jamii. Vipengele hivi vinaelezwa zaidi hapa chini.

- Uundaji wa kamati ya afya yenye wanachama 10 katika kila kambi ili kutumika kama unganisho kati ya mfumo wa afya na kuhakikisha kuwa jamii inahusika vilivyo

- Kutoa mafunzo kwa CHWs ili kutekeleza uchunguzi wa jamii wa nyumba hadi nyumba wa Kifua Kikuu ikifungamanishwa na kutoa mafunzo kwa HCWs kuhusu udhibiti wa TBna wa ugonjwa Sugu wa TB ambao hausikii dawa.

- Utathmini wa matokeo ya visa vilivyopatikana katika jamii kila baada ya miezi mitatu na HCWs. Matokeo haya yanajadiliwa na jamii kwa ujumla kupitia mkutano unaoongozwa na viongozi wa eneo/sehemu, mwakilishi wa eneo wa TBna mteuliwa wa UNHCR. Washiriki wanaokutana hukubaliana kuhusu mapendekezo ya kuboresha udhibiti wa TBkatika jamii zao.

- Mikutano ya uhamasisho na viongozi wa jamii ili kusaidia kupigana na unyanyapaa dhidi ya TBna WVU na wagonjwa wanaopata magonjwa haya.

- Msaada wa kilishe unatolewa na UNHCR kwa visa vilivyotambuliwa

- Mpango wa matibabu wa kitaifa kwa sasa unakubalisha wagonjwa wa TB kupokea matibabu wakiwa kambini hivyo kufaidika na msaada kwa jamii zao.

\section{Matokeo}

Kufuatia msaada wa kamati za afya ya jamii na viongozi, CHWs kufikia sasa wamewapa visa 140 vinavyokisiwa rufaa hadi vituo vya afya kufanyiwa utambuzi. Mchakato huu ulielekeza kutambuliwa kwa visa 12 haswa vya Kifua kikuu kufikia katikati ya mwaka - yaani mara mbili ya idadi inayotarajiwa (ya visa 6) katika kipindi kinachofikia nusu ya mwaka.

\section{Washiriki:}

Mamlaka Baina ya serikali mbalimbali kuhusu Maendeleo

Wizara ya Afya nchini Djibouti

Kwa habari zaidi, wasiliana na:

Dr. Tongna Alain Rodrigue Zoure: zoure@unhcr.org 


\section{Desturi Inayojitokeza kutoka Djibouti: Ushiriki wa Jamii katika Ukusanyaji wa Taka na Watu Husika}

1 apoa awali, shughuli ya uokotaji taka gumu kutoka kwa nyumba za wakimbizi ulifanywa na mtu mwingine ila shughuli hii iliwachiwa Watu Husika kwa sababu za kupunguzwa kwa bajeti. Katika awamu za kwanza, kampeni za uhamasisho za ukusannyaji taka uliwashirikisha wanajamii wachache. Mshiriki mmoja wa UNHCR anayeshughulikia swala hili, Direction de I'Hydraulique Rurale (DHR), hivyo alipendekeza kuanzishwa kwa mikakati ya kutoa taka kama ile inayotumika katika miji ya nchi ya Djibouti.

Kiongozi wa jamii ya wakimbizi alihusika katika kutengeneza mkakati wa kutoa taka za nyumbani pamoja na DHR, huku wakitambua maeneo mbalimbali ya ukusanyaji taka na kuhakikisha kuwa hakuna nyumba iliyosahaulika katika shughuli hii. Ushirikiano huu ulielekeza kuanzishwa kwa ratiba ya kutoa taka ya kila juma, njia itakayotumika na maeneo ya kuchukua taka hii na haya yote yaliwasilishwa kwa wanajamii. Pikipiki mbili zilinunuliwa ili kupitia njia zitakazotumika kila siku huku wakimbizi wakusubiri katika maeneo ya ukusanyaji katika maeneo yao ili kutupa taka za nyumba zao.

Ili kuhakikisha huduma bora za ukusanyaji na udhibiti wa taka za nyumbani DHRiliwateua wakimbizi kuwa watakaohamasisha usafi. Wahamasishaji wa usafi walinufaika kwa vipindi vya kukuza uwezo wao ili kuhakikisha kudumishwa kwa mazingira bora ya kuishi katika jamii zao husika.

Kudumishwa kwa usafi ambako mkakati huu unakuza pia unaweza kuwa njia ya kuzuia migogoro baina ya wenyeji na Watu Husika. Isitoshe, kwa vile viongozi wa jamii ya wakimbizi wanahusika katika kutengeneza mkakati hadi kutekelezwa na pia kusimamia shughuli yote, wao wana uhuru wa kurejelea na kubadilisha mkakati huwa kama inavyohitajika.

\section{Washiriki:}

Direction de l'Hydraulique Rurale

Office National d'Assistance aux Réfugiés et Sinistrés

Kwa habari zaidi, wasiliana na:

Moustapha Hassan Ahmed: mhaktulli@gmail.com

Abdo Ali Kayad: abkalay@yahoo.fr

\section{Desturi Inayojitokeza kutoka Ethiopia: Kupanga Uchaguzi wa Wawakilisi wa Wakimbizi Kulingana na Makadirio ya Umri, Jinsia na Uanuwai (AGD)}

ufuatia masuala ya usalama yanayotokana na uhasama kati ya makabila ambao ulisababisha kupotezwa kwa maisha ya wakimbizi katika kambi ya wakimbizi ya Kule, Shirika la Masuala ya Wakimbizi na Wanaorudi (ARRA), shirika la

usimamizi wa kambi, lilisimamisha kazi Kamati Kuu ya Wakimbizi (RCC) (ambayo ilikisiwa kuwa ilihusika) na kwa muda ikaanzisha Kamati ya Amani ambayo ilikuwa na majukumu mawili, jukumu la kutatua migogoro na la kuongoza jamii. Kulikuwa nahitaji la kuanzisha kamati mpya itakayochaguliwa na inayoaminika ya kuwawakilisha itakayochukua hatamu kutoka kwa Kamati ya Amani na kuendeleza usimamizi wa jamii ya kambi ya wakimbizi ya Kule.

Mkakati huu wenye nyenzo mbili ulihusisha kutengeneza miongozo ya uchaguzi ambayo iliwezesha uelewano kati ya washikadau na kukuza usawa, uwazi na uwajibikaji katika mchakato wa uchaguzi; Na kuwezesha kufanyika kwa uchaguzi wa wawakilishi wa wakimbizi kulingana na mtazamo wa AGD.

Huku UNHCR na ARRA wakiwezesha kukuzwa kwa miongozo ya uchaguzi na kufanywa kwa uchaguzi, wanachama wa Kamati ya Amani (wanachama watendaji wa RCC) walichangia katika kutengenezwa kwa mwongozo huu na wakaongoza mchakato wote wa uchaguzi kwa kuwafaamisha wanajamii kuhuu miongozo hii, kuhamasisha jamii katika uchaguzi na kusimamia uchaguzi katika kila eneo ndogo wakiwa kama kamati ya kusimamia uchaguzi. Kuhusika kwa Kamati ya Amani (iliyojumuisha kabila zote ndogo) kutoka kwa ukuzaji wa miongozo ya uchaguzi hadi katika uchaguzi wenyewe ulikuwa unalenga kuzuia wasiwasi wowote wa ulinzi utakaotokana na kutengenezwa kwa RCC mpya. Miongozo hii ya uchaguzi ilikuwa na sehemu ya ujumuishwa na kukinga makundi yote ya watu ikijumuisha Watu wenye Mahitaji Maalum.

\section{Matokeo}

Kutokana na desturi hii inayojitokeza, Kamati Kuu mpya ya Wakimbizi imefanikiwa kuanzishwa na ina wanachama 15 waliochaguliwa. Kwa mara ya kwanza, makabila yote na watu wenye mahitaji maalum wamewakilishwa katika muundo wa uongozi. Idadi ya wanawake katika muundo huu wa uongozi pia uliongezeka kutoka 1 hadi 5. 


\section{Washiriki:}

Shirika la Maslahi ya Wakimbizi na Wanaorudi

Kamati ya Amani ya Wakimbizi

\section{Kwa habari zaidi, wasiliana na:}

Dismas Nyamwana: nyamwana@unhcr.org

Mekdes Aschalew: aschalew@unhcr.org

\section{Desturi Inayojitokeza kutoka Ethiopia: Miongozo ya Uwezeshaji wa Jamii}

M iongozo ya Uwezeshaji wa Jamii ilitengenezwa na viongozi wa jamii ya wakimbizi nchini Eritrea kwa pamoja na UNHCR, Shirika la Masuala ya Wakimbizi na Wanaorudi (ARRA), na mashirika mengine yasiyo ya kiserikali (NGOs) ikiwa na lengo la kutoa msaada wa kifedha na mali ambayo ingewawezesha wakimbizi kujutegemea, na kuwezesha jamii kwa kukuza uwezo wake wa kutengeneza na kusimamia shughuli za ulinzi katika kambi za wakimbizi.

Kufikia hapa, Shirika la Ukarabati na Maendeleo (RADO), moja ya washiriki wa UNHCR inayotoa huduma kwa kufanya kazi na Watu wanaoishi na Ulemavu na watu wazee ilipokea bajeti ya ETB 770000 (Dola 24,062.5) ili kusaidia vyama vyake katika kuanzisha makundi ya kujisaidia binafsi kupitia Miradi ya Kuwezesha Jamii katika kambi zote nne za Tigray: Adi Harush, Mai Aini, Hitstats, na Shimelba. RADO husimamia vyama 8 (vyama viwili katika kila kambi) - vyama 4 vikiangazia Watu wanaoishi na Ulemavu na 4 vikiangazia watu wazee). Miongozo ya Kuwezesha Jamii ilitoa muundo wa Miradi ya Kuwezesha Jamii na shughuli za kufanya hivi vilijumuisha:

- Kuhamasisha watakaonufaika (hapa ikiwa ni vyama vya RADO) kuhusu miongozo wakati wamkutano na kila chama huku UNHCR ikitoa mwongozo wakati inaitajika. Vipindi vya maswali na majibu vilijumuishwa ili kuhakikisha kuwa wakimbizi walielewa miongozo hii kikamilifu na miongozo hii kutafsiriwa hadi lugha ya Tigriana kupewa wakimbizi.

- Kuandika mapendekezo ya mradi ya kila chama kwa wakimbizi wakishauriana na wanajamii kupitia fomu ya kuwezesha jamii. Vipengele vikuu katika mapendekezo vilijumuisha mahitaji, malengo na mipango ya usimamizi wa mradi.

- Kutoa msaada wa ziada katika kukuza mapendekezo kwa vyama vinavyohitaji msaada huu. Hii ilihakikisha kuwa mapendekezo yaliwasilishwa kwa muda uliofaa na pia kujumuisha mafundisho kuhusu bajeti na kupanga shughuli.

- Kutambua maeneo ya kuweka miradi kwa kushirikiana na mameneja wa kambi.

- Vipindi vya utathmini vya kila chama ili kuhakikishia ubora wa mapendekezo yao kabla ya kuwasilisha mapendekezo haya kwa kamati itakayochagua.

- Kutoa mafunzo kwa timu ya usimamizi na RADO kuhusu usimamizi wa biashara ndogo kabla ya ugavi wa pesa na mali.

- Ufuatiliaji unaofanya na timu ya pamoja (UNHCR, Kamati Kuu ya Wakimbizi, RADO) kupitia kutembelea maeneo ya mradi mara kwa mara.

- Kutoa malipo ya kila mwezi kwa wale wanaosimamia mradi moja kwa moja.

\section{Washiriki:}

Shirika la Maslahi ya Wakimbizi na Wanaorudi

Shirika la Ukarabati na Maendeleo

Kwa habari zaidi, wasiliana na:

Yamah Massaley: massale@unhcr.org 


\section{Mfano wa Kisa kutoka Kenya: Kutumia Michezo kama njia ya Kulinda na Kuzuia Vita}

$\mathrm{M}$ ichezo imekuwa na jukumu muhimu katika kukuza ulinzi kwa wanawake, wanaume, wasichana na wavulana katika sekta mbalimbali kama elimu, afya na kuishi kwa amani kaika kambi ya wakimbizi ya Kakuma na makazi ya Kalobeyei. Jambo la kushangaza ni kuwa, michezo nchini Kenya (hasa mpira wa miguu) pia inaweza kusababisha hisia kali na migogoro kati ya makundi ya kabila moja na hata utamaduni mmoja.

Ikiwa ni njia ya kusitisha vita na kukuza kuishi kwa amani kati ya wakimbizi na jamii wanaoishi katika eneo, UNHCR na washirika wenza walianzisha muundo mpya wa michezo inayojumuisha timu zenye watu wa uraia na kabila tofauti ikilenga kukuza uhusiano bora kati ya makundi tofauti ya watu wanaowakilishwa na wakimbizi na wanajamii wanaoishi katika eneo hilo.

Muundo huu mpya unakuza mabadiliko ya tabia kwa wasichana, wavulana na wanaume wachanga na wanawake wachanga pamoja na kutumia kanuni za 'Kutodhuru yeyote au chochote' kupitia shughuli kadhaa muhimu:

- Kutengeneza ligi za wanaume ('Kakuma Premier') na wanawake ('Diva League') za mira wa miguu: Ligi hizi zinahitaji kuwa timu zinazoshiriki ziwe na watu wa kabila na uraia tofauti.

- Kutoa Mafunzo ya Kitaalam ya Marefa: UNHCR na washiriki wenza walitoa mafunzo ya kitaalam kwa marefa ikijumuisha wanajamii wa eneo husika kwani iliaminika kwa vita vilichochewa na dhana ya uamuzi wa kimakosa wa marefa.

- Kutoa Mafunzo ya Kitaalam ya Makocha: Makocha pia walipokea mafunzo chini ya mkakati huu kwani wao huwa viongozi wanaoheshimika wa timu za mpira wa miguu wanaojukumika katika kukubali maamuzi (hata pale yanapoonekana kuwa ya kimakosa), na kuwatuliza wachezaji na mashabikiambao huenda hawaelewi fika maamuzi ya marefa.

- Usimamizi wa Timu na Uwanja wa Michezo: Baraza la Michezo huchaguliwa na wachezaji ili kusimamia uendeshaji wa michezo kama ilivyoainishwa katika Kakuma Premier League. Mabaraza ya Michezo na Sekretariati imetengenezwa katika kila eneo kwa kusudi hili. Wanatekeleza jukumu muhimu katika kupanga shughuli za michezo za kuridhisha umma ili kuepuka migogoro yoyote inayoweza kutokea. Wanachama wa Mabaraza ya Michezo wanapokea mafunzo kuhusu stadi za uongozi, ugavi wa raslimali na usimamizi.

- Kutengenezwa kwa 'Kakuma United Football Club': Ili kuimarisha kuishi kwa amani na kuhamasisha kupendezwa kwa mashindano yasiyoishia katika eneo moja t, UNHCR pamoja wa washirika wenza wamependekeza kutengenezwa kwa klabu itakayojumuisha wachezaji bora kutoka kwa timu zaligi ya Premier ili kushindana katika Kitengo cha Pili cha Ligi ya Kitaifa ya Mpira wa miguu nchini Kenya. Wachezaji wa Kakuma United Football Club wamepata hadhi katika maeneo yao na kujulikana zaidi kati ya wakimbizi na Wakenya.

- Programu ya Ushauri: Mafunzo ya kutoa ushauri yanafanyika yakiwalenga wasichana au wanawake wachanga, na wavulana katika mji wa Kakuma. Kupitia mikutano na wachezaji wanaojulikana na wanaoheshimika kutoka kwa Kakuma Divas League (ligi ya wanawake), wasichana na wanawake wanahamasishwa na kupewa mafunzo na stadi kuhusu masuala mbalimbali ya kujikinga. Vipindi vya mafunzo vimefanyika kupitia mtandao wakati huu wa COVID- 19 huku kukiwa na usajil wa wachezaji kadhaa.

- Jumba la Michezo la Kalobeyei: Jumba hili linajumuisha kumbi mbili kuu, yaani, Uwanja na Ukumbi. Jumba hili linalenga kutoa mazingira salama na yanayosaidia kwa wakimbizi na jamii ya eneo hili. Shughuli zitakazoendelea katika eneo hili pia zitasaidia watu wachanga kushiriki katika ulinzi na kutumika kama mabalozi wa mabadiliko chanya, kwa kuhakikisha ushiriki jumla wa wanawake, wasichana, wakimbizi walio na ulemavu na makundi mengine yanayotengwa.

\section{Washiriki:}

Shirika la Lutheran World Federation

Kwa habari zaidi, wasiliana na:

Amin Afridi: afridi@unhcr.org

Alexander Novikau: novikau@unhcr.org

Astone Ananda: ananda@unhcr.org 


\title{
Desturi Inayojitokeza kutoka Kenya: Mipango ya Utunzi wa Kijamii kwa Watu wazima wasioambatana na mtu (Ikijumuisha Wazee) Wanaoishi na Hali Kali ya Ulemavu na Magonjwa ya Kiakili
}

M kakati huu unalenga: kutambua na kukadiria watu wazima wasioambatana na mtu wanaoishi na hali kali ya ulemavu na magonjwa ya kiakili (ikijumuisha wazee) katika mazingira ya wakimbizi; kutoa mpango wa utunzi wa kijamii kwa watu kama hawa; kuhakikisha kuwa watu hawa wanapokea ulinzi na msaada mwafaka; kupunguza unyanyapaa dhidi wa watu hawa. Mkakati huu ulitengenezewa walengwa ambao walikaa kwa muda mrefu katika vituo vya mapokezi kutokana na mahitaji yao maalum. Hapo awali, watu hawa awangeweza kuhamishwa hadi kwa jamii kwani walihitaji msaada zaidi kutoka kwa jamii.

Walengwa hawa wanatambuliwa na jamii, washiriki wenza au wafanyakazi wa UNCR na kupewa rufaa hadi timu ya huduma maalum katika kitengo cha ulinzi cha UNHCR kupitia njia zilizowekwa za kutoa rufaaa baina ya mashirika katika kambi ya wakimbizi ya Kakuma. Timu hii ya huduma maalum huchunguza visa vyote vilivyopewa rufaa na kuvielekeza kwa udhibiti wa visa kwa washiriki husika huku wakiangazia mtazamo mwafaka wa mashirika mbalimbali. Mshiriki huyu hufanya makadirio kwa ushirikiano na mtu husika, familia yake na watu husika wa jamii. Kama sehemu moja ya mpango, mshiriki huyu hutambua familia ambayo inapendelea na ina uwezo wa kusaidia mtu huyu au hata msaada mkubwa wa jamii kupitia mfumo wa urafiki ( unaojumuisha majirani, marafiki, wafanyakazi wa washiriki walio wakimbizi, viongozi wa vijana na viongozi wa jamii wanaopendelea na wana uwezo wa kutoa msaada). Katika mfumo wa urafiki, vipengele vya msaada hutambuliwa na kuainishwa, kwa mfano ikijumuisha msaada wa kupika, kuosha au kuchukua mgawo.

Mchakato wa kuweka mtu mzima aliye hatarini katika utunzi wa kifamilia unajumuisha mashauriano mengi na washiriki wenza pamoja na matembezi ya nyumbani ili kuhakikisha kuwa mbinu za utunzi na uwasilishaji umeeleweka na kukubalika na mtu huyu mzima pamoja na familia inayotoa msaada. UNHCR na washiriki wenza wanatoa msaada zaidi unaohitajika ikijumuisha uhamisho, makao, bidha za kimsingi za matumizi, kuainishwa tena katika maeneo ya msaada na kuchagua mipango mbadala ya utunzi. Kufuatia uwasilishaji huu, washiriki wenza hufanya matembezi ya ufuatiliaji ya mara kwa mara, hukuza uwezo wa jamii inayohusika na kuhakikisha kuwa mtu huyu mzima aliye hatarini na familia inajulishwa jinsi ya kuwasiliana na UNHCR na/au mshiriki mwenza ikiwa kutatokea jambo lolote.

Kimuhtasari, hatua zinazochukuliwa wakati wa mkakati ni ni zifuatazo:

- Visa vya makundi yanayolengwa vinatambuliwa.

- Wafanyakazi wa shirika wanaoshughulikia visa wanahakikisha kuwa mhusika ameandikishwa na kisha kumpa rufaa hadi mashirika yanayohusika ili kuhakikisha ameshughulikiwa ipasavyo.

- Ukadiriaji unafanyika ikijumuisha ukadiriaji wa hali yake ya hatari. Mhusika anakubali kukutana na watu waliotambuliwa wanaozingatiwa.

- Uchunguzi wa mapema unafanyika ikijumuisha kukutana na mtu aliyetambuliwa, kukadiria ikiwa wanapendezwa kushiriki katika mpango huu, kutembea nyumbani kwa mtu aliyetambuliwa na kukadiria kuweza kufika na mazingira jumla ya nyumbani humo. Ikiwa mkutano wa kwanza haukufanyika nyumbani, mbinu za kumuwasilisha mhusika zinaelezwa.

- Matayarisho yanafanyika ikijumuisha kutambua ikiwa kuna mahitaji ya mipango ya uhamisho kama vile makao, bidhaa zisizo za chakula, kuchukua mgawo, kuni na kadhalika.

- Uhamisho na kuwasilisha mtu manyumbani hufanyika baada ya mkutano na mhusika na mtunzi pamoja na familia yake ikiwa wapo na kusikiliza maswali ai masikitiko yoyote.

- Ufuatiliaji hutekelezwa ikijumuisha kufanya ufuatiliaji wa mara mbili kila juma katika miezi miwili ya kwanza katika nyumba ile, kushughulikiawasiwasi wowote utakaoibuka, kufanya ufuatiliaji wa kila mwezi katika miezi mingine mitatu na baada ya hapo ikiwa inahusika kufunga kisa hiki.

\section{Matokeo}

Katika mwezi wa kwanza wa mpango huu wa utunzi wa kijamii, jumla ya watu 9 wazima wasioambatana na mtu wanaoishi na hali kali ya ulemavu au ugonjwa wakiakili ikijumuisha wazee walitambuliwa na kuunganishwa na msaada unaotolewa chini ya mpango huu.

\author{
Washiriki: \\ African Inland Church \\ Shirika la Humanity and Inclusion \\ Shirika la International Rescue Committee \\ Shirika la Jesuit Refugee Service
}

Kwa habari zaidi, wasiliana na:

Leana Podeszfa: podeszfa@unhcr.org

Alexander Novikau: novikau@unhcr.org

Caroline Jeptoo: jeptoo@unhcr.org

Astone Ananda: ananda@unhcr.org 


\section{Mfano wa Kisa kutoka Kenya: Kuzuia Uchafuzi wa Maji kutumia Vifuniko vya Nyuzi Kufunika Jerikeni}

erikeni za ukubwa tofauti husambazwa mara mbili katika mwaka katika kambi ya wakimbizi ya Dadaab. Mpango wa muda wa kubadilisha jerikeni pia upo huku ukikubalisha ubadilishaji wa jerikeni zilizovunjika kulingana na kuwepo kwa jerikeni zilizo sawa.

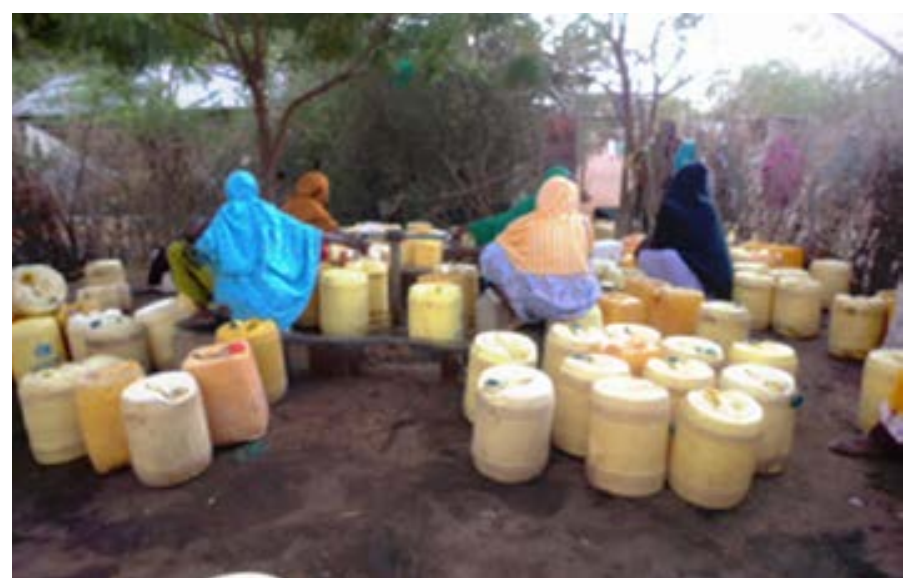

Mara nyingi jerikeni husafirishwa kutoka maeneo ya mifereji hadi manyumbani kwa kutumia wilibaro ikiwa zinapatikana na hali ya ardhi ikakubalisha matumizi yake. Ingawa jumbe za uhamasisho wa usafi hazipendekezi njia hii, usafirishaji wa jerikeni mara nyingi huhusisha kubingirisha jerikeni ile ardhini. Njia hii ya usafirishaji unahitaji jerikeni iwe na kifuniko kilichobanwa ili kuhakikisha maji hayamwagiki na yanafikishwa manyumbani kwa urahisi. Ili kufanya hivi, wakimbizi katika Dadaab wamevumbua jinsi ya kubana vifuniko kwenye jerikani. Hii ni kwa kufunga uzi kwenye kifuniko cha jerikeni hivyo kuzuia maji kumwagika na kuepukana na viini licha ya mchanga na matope ambayo yatajikusanya jerikeni inapobingirishwa.

Washiriki: haihusiki

Kwa habari zaidi, wasiliana na:

Osman Yussuf Ahmed: ahmedos@unhcr.org

\section{Mfano wa Kisa kutoka Kenya: Kamati za Maji Sokini}

mata uhakikisha ongezeko la ugavi wa maji au kudumisha ugavi huu katika mazingira ya wakimbizi kunasalia kuwa lengo muhimu. Kamati za Maji Sokoni (MWCs) zilianzishwa katika kambi za wakimbizi za Dadaab ili kubainisha maji ya matumizi ya nyumbani na ile ya 'biashara' katika jamii za wakimbizi na kuanzisha na kuendeleza mkakati wa 'kulipia huduma'. Kamati za Maji Sokoni ni biashara ya pamoja ya wakimbizi na wanajamii wa eneo hili katika kambi tatu. Lengo la muda mrefu la mkakati huu ni kubadilisha usimamizi wa huduma ya maji kwa wakimbizi hadi kuwa kampuni ya huduma ya maji inayosimamiwa na wawakilishi wa washikadau sawia na mifumo ya maji katika mijini nchini Kenya. UNHCR na Shirika la CARE imeanzisha jaribio la mradi wa 'kioski cha Maji ya ATM'wa MWC. Ikiwa jaribio hili litafanikiwa, kioski cha Maji ya ATM kitakuwa kitangulizi cha ugavi wa maji mengi ya kila mwezi kwa familia ya takriban watu 10. Familia ya ukubwa huu itapatiwa lita 20 ya maji kwa kila mtu kila siku hivyo kuakisi kuwa lita 6,000 kwa kila mwezi. Kiasi kilichowekwa cha maji kitaweza kuchukuliwa katika kioski cha Maji ya ATM wakati wowote kwa siku. Matumizi ya maji yanayopita kiwango hiki ni lazima yalipiwe.

\section{Washiriki:}

Shirika la CARE

Kwa habari zaidi, wasiliana na:

Osman Yussuf Ahmed: ahmedos@unhcr.org 


\title{
Desturi Inayojitokeza kutoka Kenya: 'Muunganisho wa Kiroho' - Makanisha ya Mjini Yanayoongozwa na Wakimbizi kutumika kama Raslimali
}

\author{
I amii za Watu Husika nchini Kenya katika maeneo ya mijini wanahitaji mbinu za kujikimu ili kuweza kupigana na \\ changamoto za maisha ya mjini katika nchi geni. Imani inaweza kuchangia katika kukuza ulinzi wa kijamii na kusulihisha \\ migogoro huku ikisaidia katika kudumisha hali ya kujijua kati ya wakimbizi.
}

Mjini Nairobi, baadhi ya wakimbizi wanatengeneza mahali pao pa kufanya ibada. Vituo hivi vya ibada vimetengenezwa kimsingi ili kuafikia mahitaji ya kiroho ya wakimbizi huku vikiiga mazoea ya kanisa za mataifa asili ya wakimbizi hawa. Moja ya kanisa kama hili viungani vya mji wa Nairobi (maeneo ya Kitengela) inaongozwa na padri ambaye ni mkimbizi na pia ni kiongozi wa jamii ya wakimbizi. Kanisa linawaleta pamoja washirika wakimbizi ambao wanawezakufikilia huduma mbalimbali ikijumuisha makao kwa wakimbizi wanaowasili katika eneo hilo; kujulishwa kwa wakimbizi wageni kwa wakimbizi wengine na mitandao; msaada wa chakula na bidhaa zingine (licha ya raslimali finyu); na mafunzo ya kimsingi ya lugha ambayo inaboresha mawasiliano na hali ya maisha.

Kanisa hili pia ni mwanachama katika 'Ujamaa wa Mapadri wa Kitengela,' jukwaa ambalo linatoa fursa na kuingiana namakanisha ya jamii ya eneo hili na kupitia hili, migogoro baina ya wakimbizi na wanajamii wa eneo hili imesuluhishwa.

\begin{abstract}
Matokeo
Makanisa ya wakimbizi inawapa wakimbizi hawa kujuhisi wanakubalika, jambo ambalo lina umuhimu katika saikolojia ya binadamu Muungano kati ya makanisa ya wakimbizi na ya jamii ya eneo hili hufungua fursa za mawasiliano na msaada kwa Watu Husika. Pia, inatoa suluhu za kiutendeti za baadhi ya changamoto ambazo wakimbizi wanapitia kuhusiana na serikali ya maeneo na polisi. Muingiliano baina ya kanisa la wakimbizi na makanisa ya jamii la eneo hilo pia unaboresha uwezo wa wakimbizi kufikia habari na nambari muhimu za msaada wakati wa mlipuko wa COVID- 19.
\end{abstract}

Washiriki:

Kikundi cha Utendakazi wa Ulinzi wa Kijamii

Sekretariati ya Masuala ya Wakimbizi

Kwa habari zaidi, wasiliana na:

Jackson Karugu: karugu@unhcr.org

\section{Mfano wa Kisa kutoka Kenya: Kamati za Jamii za mpango wa WASH}

K

ambi ya wakimbizi ya Kakuma na makazi ya Kalobeyei ziko katika maeneo kavu, haliya juu zaidi ya joto, mito inayovunja kingo zake uhaba wa jumla wa maji. Ili kuwezesha jamii kumiliki masuala yanayohusiana na mpango wa WASH, kila jamii kubwa mjini Kakuma hutengeneza Kamati ya mpango wa WASH.

Kamati za mpango wa WASH ni vikundi vya watu wanne hadi sita wanaojitolea amabo wanajukumika katika kusimamia masuala yoyote yanayoibuka katika sekta ya mpango wa WASH. Isipokuwa viongozi wawili wa jamii kubwa ambao ni wanachama wa Kamati kubwa ya mpango wa WASH, uanachama wa hao wengine huamuliwa kupitia majadiliano na wanajamii. Kwa kawaida, wanachama wanaochakuliwa huwakilisha makundi tofauti ya wanajamii kama vile wanawake, vijana, walio wachache, watu wenye mahitaji maalum na watu wanaoishi na ulemavu.

Katika maeneo ya Kakuma na Kalobeyei, maji husambazwa kupitia visima 19 kupitia mfumo wa tanki za chuma zilizowekwa juu na mfumo wa uenezaji kufikia maeneo ya mifereji katika jamii. Visima hivi hutumia nishati za jenereta na pia nishati ya jua pale inapowezekana.

Msongamano na masaa mengi ya kungoja foleni katika maeneo ya kuchota maji mara nyingi huwafanya wachota maji kufadhaika. Migogoro katika maeneo ya kuchota maji inaweza kusababisha wasiwasi na kujitenga kwa jamii. Ili kukabiliana na hili, Kamati za mpango wa WASH zinatengeneza ratiba ya uenezaji maji kupitia mazungumzo na jamii. Hivyo, kila familia inapewa mzunguko mmoja kati ya tatu ya uenezaji wa maji. Maji huenezwa mara tatu kwa siku (mara moja wakati wa asubuhi, wakati wa mchana na jioni kabla ya giza kuingia). Uenezaji wa maji kabla ya giza kuingia huhakikisha usalama wa wanawake na wasichana ambao mara nyingi jukumu lao uwa ni la kuchota maji.

Wanajamii waliochaguliwa hupewa mafunzo ya kukarabati vifaa vya mpango wa WASH, ikijumuisha maeneo yenye mifereji ambayo mara nyingi huharibika. Kuhusisha jamii katika kushughulikia na kusuluhisha masuala yao binafsi yanayohusiana na mpango wa WASH, huboresha kukuza jamii na kuimarisha uwezo wao. 
Baraza la Wakimbizi la Norway (NRC) hutoa wafanyakazi wa kitaifa wa ulinzi ili kusaidia Kamati za mpango wa WASH na kuhakikisha kuwa miradi ya WASH imetekelezwa kulingana na viwango, kanuni na miongozo ya ulinzi na kutilia mkazo kusuluhisha migogoro na kutunza miradi. Sekta ya mpango wa WASH ya NRC hufanya kazi kwa karibu na idara za UNHCR (ulinzi, kitengo cha jamii, kitengo cha mashinani, afya, elimu) ili kushughulikia masuala yoyote ya ulinzi yanayohusiana na mpango wa WASH. Kujumuishwa haswa na mkazo unatiliwa kwa nyumba zinazoongozwa na wanawake, watoto wasioambatana na mtu mwingine, wazee na Watu wenye Mahitaji Maalum. NRC linahusisha jamii katika awamu zote za udhibiti wa mfumo wa mradi wa WASH. Timu ya mpango wa WASH ya NRC hushiriki katika mkutano wa kila mwezi wa viongozi wa jamii na washiriki wenza ili kutoa maoni na kuwasilisha habari kwa jamii. Baraza la Wakimbizi la Norway pia ina mbinu ya kupata maoni, malalamiko na majibu ambayo huhimiza mawasiliano katika jamii.

\section{Washiriki:}

Baraza la Wakimbizi la Norway

Kwa habari zaidi, wasiliana na:

Oscar Nabiswa: nabiswa@unhcr.org

Moe Miyahara: miyahara@unhcr.org

\section{Mfano wa Kisa kutoka Rwanda: Magavana wa Vijiji}

I apo awali katika kambi ya Mahama, ripoti za UNHCR zilikuwa chache na zinazoegemea mazungumzo na wakimbizi, kutolewa kwa ripoti za washiriki wenza na ufuatiliaji wa mara chache na uhaba wa wafanyakazi. Hitaji la kuwahusisha wakimbizi kwa makusudi zaidi lilikuwa wazi wakati wa kuanza kwa hali ya dharura katika kambi ndipo ilipotambulika kuwa wakimbizi walio mashinani walikosa habari ya jinsi ya kusuluhisha matatizo yao binafsi na ni wapi wanaweza kuenda kupata huduma katika kambi ya Mahama. Uongozi wa UNHCR ulitengeneza mkakati wa'Magavana wa Vijiji'ili: kuhakikisha Watu Husika wanaweza kufikia habari sahihi na kwa muda unaofaa; kuimarisha uwezo wa wakimbizi katika kusuluhisha matatizo madogo wao wenyewe katika jamii bila kuingilia kati kwa washiriki wanaotoa uduma; na kufuatilia utoaji wa huduma na washiriki kupitia akaunti za jamii, kuwawajibisha washiriki wenza wakati utofauti unatokea.

Mkakati wa Magavana wa Vijiji unajumuisha kutoa mfanyakazi mmoja wa UNHCR ili kusimamia kijiji kimoja katika kambi ya wakimbizi ya Mahama, ambayo kwa takriban ina familia 250-480. Wafanya kazi hawa wanajukumika kuhakikisha kuwa wakimbizi wanaoishi katika vijiji wana ufahamu wa kutosha kuhusu huduma zinazotolewa katika kambi, wanapokea huduma kadhaa zilizoko na wana uwezo wa kusuluhisha migogoro midogo ya jamii (au kuweka mbinu za kufanya hivi ).

'Magavana" hawa (jina la kiutani) hufanya nikutano mingi na viongozi wa jamii, wakishirikiana nao katika kukusanya umma katika mikutano mara moja kwa mwezi au kila baada ya miezi mitatu. Vipindi hivi vya mikutano ya jamii hutumika katika kueneza habari kuhusu huduma zilizoko, masharti ya kambi, usalama na ulinzi, suluhu za kudumu, ulinzi wa watoto na SGBV, na jumbe za kuhamasisha jamii za kutoa suluhu za matatizo ya jamii bila kuhusisha mashirika. Vipindi vya maswali na majibu hufanyika katika mikutano hii.

Wakati mwingine magavana hawa huwaalika maofisa wa serikali na washiriki wenza ili kuwasilisha jumbe muhimu au kutoa habari kuhusiana na changamoto zinazowasilishwa na jamii. Magavana pia wanashiriki katika kazi ya jamii pamoja na wanakijiji kabla ya mikutano ikiwa ni njia ya kuhimiza kazi ya jamii na kuashiria umoja. Mara kwa mara, magavana hawa hufanya mikutano ya dharura ikiwa kuna njia mpya ya kutoa msaada, au ujumbe wa dharura unapaswa kufikishiwa wanakijiji (kwa mfano kuanzishwa kwa mikakati inayohusisha kusambaza pesa, kugawa wa gesi, habari ya shughuli ya utathmini).

Ripoti kutoka kwa mikutano hii huwasilishwa kwa timu ya usimamizi ya UNHCR, hivyo kusaidia mitindo kama hii kutambuliwa, kukusanya mafunzo muhimu na mikakati kutolewa inapohitajika.

Washiriki: haihusiki

Kwa habari zaidi, wasiliana na:

Emilia Lobti: lobti@unhcr.org; rwaki@unhcr.org 


\section{Desturi Zinazojitokeza kutoka Rwanda: Kliniki za Ulinzi ya Kuhamahama}

M ikutano ya uratibu ya kila juma kati ya UNHCR na washiriki wenza katika kambi ya wakimbizi ya Mahama (kwa ajili ya kuratibu shughuli zote za ulinzi) ilifunua hitaji la kutumia muda zaidi katika jamii ya wakimbizi, na kuhakikisha kuwa Watu Husika walikuwa wanapokea jumbe sahihi, walielewa njia ya kutoa rufaa ya visa vya ulinzi wa watoto na visa vya SGBV na kupata fursa ya kuuliza maswaliyanayohusiana na masuala ya ulinzi katika kambi. Kufanya kazi kwa pamoja kufikia hapa kuliamuliwa kuwa mkakati bora zaidi wa kujiwasilisha katika jamii na kutoa majibu ya masuala kwa njia ya mapatano. Hivyo, ratiba ya mzunguko ilitengenezwa na UNHCR ya kaziu ya washiriki katika kuratibu na kutengeneza matembezi kwa jamii ya pamoja kwa ushirikiano wa karibu na viongozi wa jamii katika majuma matatu ya kwanza ya kila mwezi. Juma la mwisho la mwezi lilitengwa kuwa la kupanga mkutano wa uratibu wa Ulinzi na kuhakikisha ushiriki wa viongozi wa washiriki na jamii.

Kutokana na juhudi hizi, kila siku ya Alhamisi, mkutano wa kijiji chote hupangwa katika jamii, na mahali pa mkutano kuzunguka katika vijiji vyote 18 vya kambi ya Mahama na kuongeza mashule, maeneo ya soko na jamii inayoishi katika eneo hilo. Mikutano hii hupangwa kwa pamoja na UNHCR, Wizara Inayohusika na Udhibiti wa Dharura (MINEMA) na washiriki wanaohusika na Ulinzi kwa ushirikiano na viongozi wa vijiji na wa jamii, miundo ya kijamii ya Maslahi ya Jamii (makundi yanayopinga SGBV, makundi ya vijana, watetezi wa jamii, makundi ya uigizaji, makundi ya usakataji densi). Desturi hii inafahamika kama'Kliniki ya Ulinzi ya Kuhamahama'.

Desturi ya Kliniki ya Ulinzi ya Kuhamahama kwa kawaida hutanguliza kumtambulisha kiongozi wa kijij/jamii, na kufuatiwa na densi, wimbo, shairi au uigizaji kuhusu swala fulani kuhusu ulinzi katika jamii. Washiriki hutoa maonyesho yao kuhusiana na huduma zinazopatikana na majibu ya njia ya/ hatua za kujilinda kutokana na uhalifu, ulinzi wa watoto na masuala ya SGBV.

Desturi ya Kliniki za Ulinzi ya Kuhamahama inatoa fursa kwa UNHCR, Wizara Inayohusika na Udhibiti wa Dharura na washiriki wenza kuhamasisha jamii kuhusu mada tofauti tofauti za kujilinda na kueneza habari za hivi punde zaidi na matangazo. Kipindi cha maswali malalamishi na kutoa maoni kwa jamii kinajumuishwa katika mkakati huu sawia na majibu kutoka kwa washiriki.

Visa vya watu binafsi na zile nyeti hazizungumziwi kamwe katika matembezi haya. Badala yake, washiriki hutoa habari ya kijumla ya ni wapi mtu anaweza kufikia taasisi tofauti ikiwa anahitaji, na visa vya watu wanaojitambulisha wakati wa vipindi hivi hupewa rufaa hadi kwa washiriki wanaohusika. Jamii inazidi kukumbushwa kuhusu haki zao, wajibu wao na kuheshimu masharti na kanuni ya kambini.

Kliniki hii ya kuhamahama hufanyika kwa njia inayoshirikisha na kupendeza huku viongozi wa jamiii ya washiriki wakitumia lugha yenye heshima na ya kukuza jamii. Lugha inayowajali watoto pia hutumika wakati wa kuzungumzia watoto na ushiriki wa wanawake, wanaume, wasicana na wavulana kutoka kwa asili zote hupendekezwa kupitia uhamasisho.

\section{Matokeo}

Kliniki za Ulinzi za Kuhamahama ni njia ya kuweza kufikia jamii kinyume na kutarajia wanajamii kuwatafuta washiriki husika. Kupitia desturi hii, washiriki wanaweza kufahamu ukweli kuhusu mambo ambayo wakimbizi hupitia, kupokea malalamiko na maoni mbalimbali pamoja na fursa ya kutoa yao majibu kwa jamii.

\section{Washiriki:}

Wizara Inayohusika na Udhibiti wa Dharura

Shirika la Save the Children International

Shirika la Alight

Shirika la Adventisti la Maendeleo na Kutoa Msaada

Shirika la International Committee of the Red Cross

Legal Aid Forum

Prison Fellowship Rwanda

Kwa habari zaidi, wasiliana na:

Emilia Lobti: lobti@unhcr.org 


\section{Desturi ya Kutia moyo kutoka nchi ya Rwanda: Vyama vya Kuweka Akiba kwa Hiari na Mikopo}

ufuatia mashauriano na viongozi wa jamii, wakimbizi wanaoishi na ulemavu na familia zao katika kambi za wakimbizi
nchini Burundi, changamoto wanazopitia wakimbizi walio na ulemavu ziliweza kujitokeza. Zile zilizojitokezazilijumuisha
ufikiaji adimu wa huduma zilizoko za hali ya maisha, kuwa na uwezekano wa kuwa hatarini ya kudhulimiwa na kutumika
vibaya na uzoefu wa unyanyapaa na kubaguliwa. Matokeo haya yaliainika na shughuli kuu ya UNHCR ya Umri, Jinsia na
Uanuwai, kutambua ubaguzi ambao wakimbizi wenye ulemavu hupitia sawia na maswali yanayojitokeza mara kwa mara
kuhusu ulinzi/usalama na mapengo yaliyoko katika kutoa huduma. Vyama vya Vijijini vya Kuweka Akiba na Mikopo (VSLAs)
vinavyosisitiza kujumuishwa kwa wakimbizi wenye ulemavu vilianzishwa kutoka na hali izi halisi ikiwa kama njia ya kukuza
kujitegemea kwa kikundi hiki na kujumuishwa katika miradi.

Kwa ushirikiano na washiriki wanaotoa huduma ya ulinzi katika jamii, UNHCR lilitengeneza shughuli kadhaa zilizolenga kukuza ujithamini na ujistahi wa Watu wanaoishi na Ulemavu, ikijumuisha kuhamasisha watu katika kambi ya Mahama na wenyeji kwa mujibu wa Kanuni 7 za Kijumla za Muungano wa Umoja wa Mataifa kuhusu Haki za Watu wanaoishi na Ulemavu ambazo ni:

- Heshima kwa adhama asilia

- Uhuru wa kibinafsi ikijumuisha uhuru wa kuchagua na kujitawala; bila ubaguzi

- Ushiriki kamili na ulio bora na kujumuishwa katika jamii

- Heshima kwa utofauti na kukubali Watu wanaoishi na Ulemavu kama uanuwai wa binadamu na utu;

- Kufikia nafasi kwa usawa

- Upatikanaji

- Usawa kati ya wanaume na wanawake

Vipindi hivi vya uhamasisho viliboresha ufahamu wa Watu wanaoishi na Ulemavu kuhusu haki na uhuru wao kushiriki kikamilifu katika jamii. Watu wanaoishi na Ulemavu hivyo walitengeneza kamati ya washiriki ambayo ilitoa msaada huku wakianza kutengeneza shughuli zao binafsi za kuleta mapato ikijumuisha Vyama vya Kuweka Akiba kwa Hiari na Kutoa Mikopo (VSLAs), ili kupunguza jinsi wanavyotegemea msaada na kuongeza ushiriki wao katika shughuli za kijamii na kiuchumi.

Ili kuwezesha uendelevu wa vyama vilivyotengenezwa, UNHCR kwa ushirikiano na shirika la Human Inclusion ilianzisha vipindi vya kukuza uwezo wa vyama katika mada zifuatazo, uongozi wa vyama; ushiriki na majukumu ya kila mwanachama katika chama; jinsi ya kuanzisha masharti na kanuni katika vyama;sharti la kuweka malengo, Kufuatilia na kutathmini katika chama; kuweka kumbukumbu; stadi za kuweka akiba; kudhibiti akiba na kukuza mikopo; uhasibu na mapendeleo ya kufanya kazi na taasisi za kifedha. Vyama hivi vilihimizwa kufungua akaunti katika taasisi ndogo za kifedha ili kuboresha uwezekano wa kupata mikopo ya benki kama suluhu za kudumu za kiuchumi.

UNHCR na washiriki wenza inatekeleza vipindi vya mafunzo na kufanya mikutano ya mara kwa mara na vyama hivi na kuhakikisha vinafuata kanuni ya 'Kutodhuru Yeyote na Chochote', na kuwezesha waanzilishi wa chama kwa njia isiyo ya ubaguzi. UNHCR pia hufuatilia kuzingatiwa kwa masharti ambayo VSLAs waliyakubali, kuwa na kipengele cha uwekaji akiba katika mikakati yao na wanamakinika katika kuboresha kujitegemea, kujikuza na hadhi yao. Mikutano ya mara kwa mara kati ya washiriki wanaohusika na wanaonufaika, na msaada wa uhasibu, pamoja na mikakati mingine huhakikisha kuwa vyama hivi vina mfumo wa kufuatilia na kuwajibika ili kuhakikisha uwazi.

\section{Matokeo}

- Kambi ya wakimbizi ya Mahama kwa sasa ina vyama 15 ambavyo vimetengenezwa hasa na Watu wanaoishi na Ulemavu ila ushiriki uko wazi kwa wanajamii wote.

- Wanajamii ikiwajumuisha viongozi, wameunga vyama vya kuleta mapato vilivyoanzishwa na Watu wanaoishi na Ulemavu. Katika kambi ya wakimbizi ya Mahama, VSLAs vyote vikiunganishwa vina wanachama 361 (159 wakiwa nia wa kiume na 202 wakiwa ni wa kike). Kati ya wanachama hawa, 90 ni wa kikundi cha Watu wanaoishi na Ulemavu.

- Jumla ya VSLAs 15 vimefungua akaunti kwenye benki.

- VSLAs kwa sasa hutafutwa na washikadau mbalimbali (kwa mfano sirikali ya Rwanda na ubalozi wa kigeni) ili kushiriki katika mashindano, maonyesho na sherehe za kutuza kambi na katika matukio mengine maalum. Katika mikutano ya umma na siku mbalimbali za kimataifa, muda fulani hutengewa wakimbizi wanaoishi na ulemavu, familia zao na washiriki ili kushuhudia jinsi VSLAs vimebadilisha maisha yao.

- Jamii hushuhudia kuwa wakimbizi wanaoishi na ulemavu pia wana uwezo na nguvu ya kusaidia familia zao na jamii kwa ujumla. 
Washiriki:

Shirika la Humanity and Inclusion

Kwa habari zaidi, wasiliana na:

Emilia Lobti: lobti@unhcr.org; rwaki@unhcr.org

\title{
Desturi Zinazojitokeza kutoka Uganda: Kuboresha Sauti za Wakimbizi kupitia Majadiliano Yanayoshirikisha Wakimbizi
}

\begin{abstract}
kiwa nchi yenye idadi ya juu zaidi ya wakimbizi barani Afrika na nchi za kwanza kutekeleza Mfumo wa Kina wa Mwitikio kwa Wakimbizi (CRRF), nchi ya Uganda ilipitisha Ramani yake ya Utekeleza wa CRRF katika mwezi wa Januari 2018. Uganda ilitoa maono ya kuimarisha kutekelezwa kwa mtazamo wa kina katika kuitikia ili kusaidia kupunguza shinikizo kwa wilaya wanazokaa wakimbizi na kufikia mahitaji yao pamoja na ya wenyeji. Ramani ya Utendakazi wa CRRF ya Uganda inatambua kufikia mahitaji ya wakimbizi na wenyeji kama mojawapo ya vipengele vikuu katika CRRF nchini.
\end{abstract}

Kikundi cha Uendeshaji (SG) wa CRRF ni bodi ya kiwango cha juu cha kutoa maamuzi na hukutana kila baada ya miezi mitatu ili kuendeleza utekelezaji wa CRRF kimkakati nchini Uganda. CFFR SG ni jukwaa maalum inayoleta pamoja wizara, idara na mashirika ya serikali, washiriki wa kimataifa wenye mahusiano ya pande mbili na yenye mahusiano mengi, NGOs za kimataifa na za eneo, sekta ya kibinafsi, wakimbizi na wenyeji. Viti viwili vya Kikundi cha Uendeshaji wa CFFR hutengewa wakimbizi ili kuwakilisha wakimbizi milioni 1.4 nchini humo.

Majadiliano Yanayoshirikisha Wakimbizi (REF) yalianzishwa mwezi wa Oktoba 2018 ili kuhakikisha kuwa jamii zilizoathirika zimewakilishwa vyema katika CFFR SG. Kabla ya REF kuanzishwa, miundo ya uongozi wa wakimbizi ilifika katika kiwango cha makazi (Mabaraza ya Maslahi ya Wakimbizi) haikuwa na ushirikishi wa kimuundo katika kiwango cha kitaifa. Kutokana na kizuizi hiki, usirikishi wa washikadau na jamii ya wakimbizi katika kiwango cha kitaifa ulikuwa wa dharura na usio na mfumo wowote. Kwa sasa, REF ina wanachama 37 kutoka kwa uongozi wa makazi ukiakisi makundi anuwai ya kidemografia, kijinsia na umri. Hii ilijumuisha viongozi wawili waliochaguliwa kutoka kwa kila makazi, wawakilishi wa vijana na viongozi wa jamii mjini Kampala. Wanachama wa REF huchagua watu wawili kutoka kwa kikundi hicho - mmoja wa kike na mwingine wa kiume wa uraia tofauti na makazi tofauti - ili kuwakilisha wakimbizi katika mikutano ya SG inayofanyika kila baada ya miezi mitatu kwa muda wa mwaka mmoja. Mikutano ya REF hufanyika kila baada ya miezi mitatu kabla ya mikutano ya ya kila baada ya miezi mitatu ya CFFR SG ili kueleza CFFR SG kuhusu maamuzi yote yanayowaathiri wakimbizi. Wawakilishi wawili kutoka kwa REF wamehudhuria mikutano yote ya CFFR SG kutoka mwezi wa Oktoba 2018. Kupitia REF, jamii ya wakimbizi pia imekuwa na habari kuhusiana na majadiliano na maamuzi ya CFFR SG.

Jopokazi la Majadiliano Yanayoshirikisha Wakimbizi hupanga na kurahisisha mikutano ya Majadiliano Yanayoshirikisha Wakimbizi. Jopokazi hili linajumuisha Ofisi ya Waziri Mkuu (OPM), Sekretariati ya CFFR, UNHCR na INGOs (mashirika ya CARE, Oxfam, IRC na mengineyo). Makamanda wa Makazi na Idara za Wakimbizi ya maeneo chini ya Ofisi ya Waziri Mkuu hufanya kazi kwa karibu na ofisi ndogo au za mashinani za UNHCR ili kusaidia kazi ya wanachama wa REF ya kuwasilisha maoni kwa jamii kuhusu yaliyojadiliwa katika mikutano ya REF na kushauriana na jamii kuhusu wanayopenda yawasilishwe kwa REF na CFFR SG.

Majadiliano Yanayojumuisha Wakimbizi hukubalisha ushiriki wa jamii ya wakimbizi katika kupanga, kutekeleza na kufuatilia CFFR nchini Uganda. Muundo wa uongozi wa wakimbizi upo kwa sasa kutoka kwa kijiji - eneo- makazi - kitaifa ili kusaidia kufanya majadiliano katika viwango vya mashinani hadi kitaifa na kitaifa hadi mashinani. REF sio mbinu muhimu tu ya uwajibikaji kwa washikadau wote katika mwitikio wa wakimbizi; pia huchangia kuboresha kujitegemea kwa wakimbizi, kwa kushughulikia mahitaji ya wakimbizi na ya wenyeji kikamilifu.

Wawakilishi wawili wa Wakimbizi kutoka kwa REF wamehudhuria GRF, IGAD, TICAD na mashirikisho mengine ya kimataifa ili kueleza uzoefu na ufahamu wao na kujadili masuala yanayoathiri jamii yao.

Washiriki: mbalimbali

Kwa habari zaidi, wasiliana na:

Yoko Iwasa: iwasa@unhcr.org

Leslie Velez: velez@unhcr.org

Ndahiriwe Innocent: indahiriwe7@gmail.com

Rick Bartoldus: Rick.Bartoldus@rescue.org

Annet Kyambadde: Annet.Kyambadde@care.org 


\section{Desturi Zinazojitokeza kutoka Uganda: \\ Kuanzishwa kwa Ofisi za Baraza la Maslahi ya Wakimbizi Zinazoongozwa na Jamii, Kutengenezwa kwa Makundi ya Msaada kwa Watu wenye Mahitaji Maalum na Mkakati wa Kukuza Stadi}

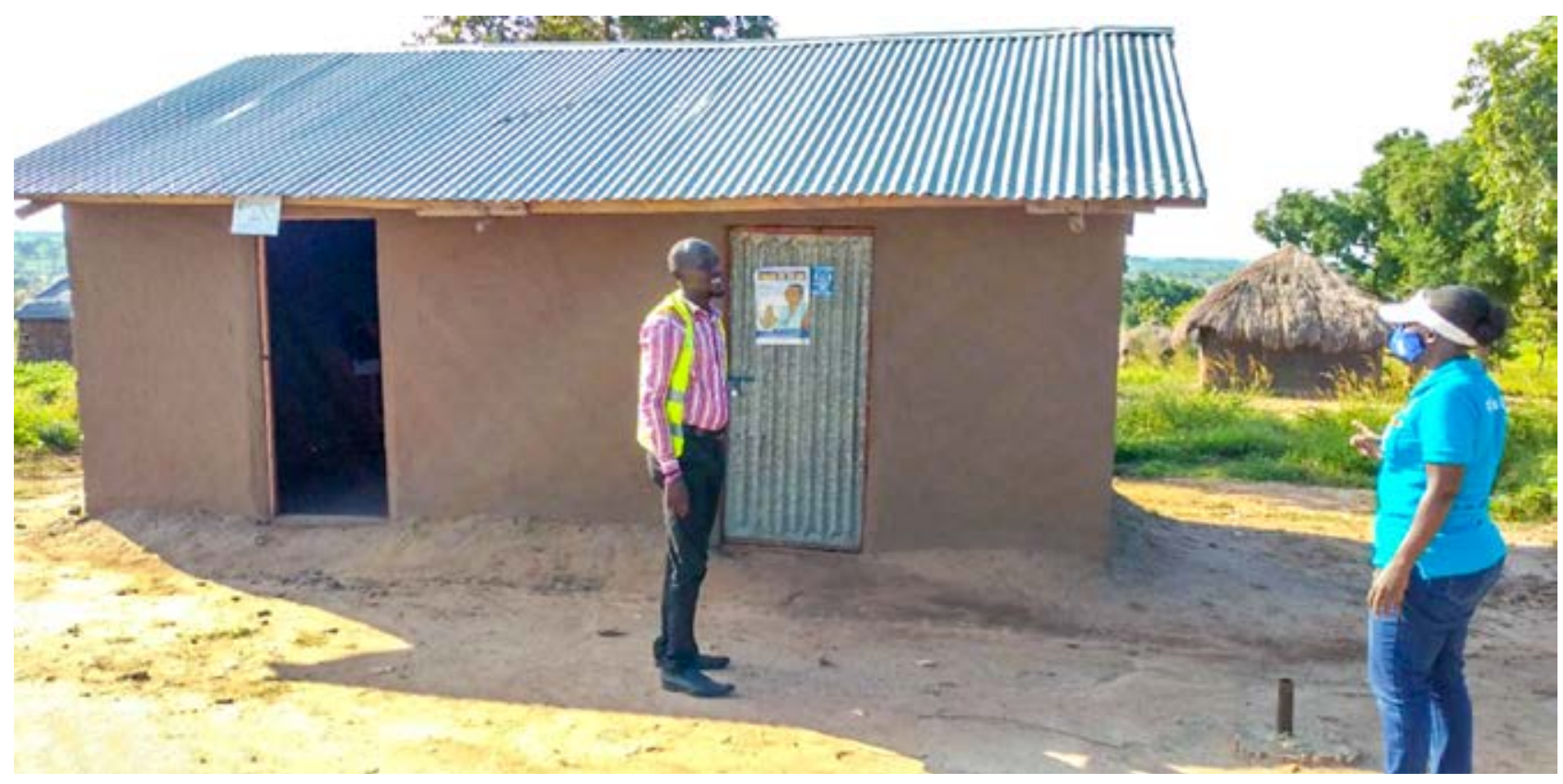

K ujengwa kwa maeneo yasiyo ya kudumu ya Baraza ya Maslahi ya Wakimbizi (RWC) katika Eneo la Ofua kulikuwa ni mkakati ulioongozwa na jamii pekee kikiongozwa na viongozi wa RWC na kuangazia kuboresha uwezo wao wa

kushirikiana na washiriki na jamii kwa ujumla kwa ubora zaidi. Maeneo ya ofisi ya Baraza ya Maslahi ya Wakimbizi yalionekana kutengeneza mazingira mema na ya faragha katika ushirikiano kati ya wanajamii, washiriki na RWCs, huku yakiboresha ushiriki wa jamii, kudumisha uwazi na uwajibikaji, na kuzingatia mbinu za kutoa rufaa zilizopo.

Maeneo ya ofisi ya RWC yameanzishwa katika Vijiji 6 vya Eneo la Ofua. Maeneo haya yamerahisishia wakimbizi na washiriki katika kuwafikia viongozi wa RWC kupata msaada na kuwahusisha katika kupanga, kutekeleza na kufuatilia huduma zinazotarajiwa. Maeneo haya huwa wazi wakati wote huku kukiwa na mwanachama mmoja wa utekelezaji wa RWC atakayeshughulikia masuala ikijumuisha malalamiko au wasiwasi, kutoa maoni na hitaji la kuwepo kwa mahali pa kufanyia mikutano.

Isitoshe, Watu wenye Mahitaji Maalum katika Eneo la Ofua wameanzisha makundi sita ya msaada inayofanya kazi (kikundi kimoja katika kila kijiji) na kikundi kingine cha saba (kinachojumuisha wanachama 19 wanaowakilisha eneo lao) kuchaguliwa katika kusimamia makundi haya sita ya kijamii. Makundi ya msaada kwa Watu wenye Mahitaji Maalum hukutana mara kwa mara kujadili masuala yanayowaathiri na kubuni suluhu za kushughulikia masuala haya. Kila kikundi kina mwenyekiti, naibu mwenyekiti, mweka hazina na katibu wa kuratibu changamoto na mafanikio ya kikundi. Makundi haya ya msaada yanatumika kama mbinu ya: kusaidia kukuza ufahamu wa Watu wenye Mahitaji Maalum kuhusu haki na wajibu wao, kutoa ushauri wa kimsingi kwa Watu wenye Mahitaji Maalum wanaohitaji na kujumuisha Watu wenye Mahitaji Maalum katika fursa za kujikimu kimaisha (kama vile, kufuga kuku, kuweka bustani ya jikoni), na katika biashara ndogo za kuuza na kuweka akiba.

Mwishowe, wanawake katika kijiji cha Ofua II walijipanga katika kikundi cha wanachama 30 kujifunza stadi za kujikuza kama vile kushona nguo, kutengeneza shanga/kufuma/kusuka, kutengeneza viatu, kusuka nywele na kutengeneza sabuni. Makundi haya ya wanawake yanatoa mafunzo ili kuwapa wanawake wasio na uwezo wa kusoma na kuandika katika jamii stadi za kuwawezesha kujikimu kimaisha.

\section{Matokeo}

Watu Husika wana imani na viongozi wao na wanazidi kuwasilisha wasiowasi wao kupitia miundo iliyowekwa. Imeonekana kuwa viongozi wa wakimbizi wanahusika katika kufanya maamuzi ili kusuluhisha mambo yanayowakumba wao na jamii yao pamoja na kuonekana kwa viongozi hawa katika jamii kupitia maeneo yaliyoanzishwa. 
Makundi ya msaada kwa Watu wenye Mahitaji Maalum yamechangia na kuweka pesa kama sehemu ya Chama cha Kijijini cha Kuweka Akiba na Mikopo. Pia wanaendelea kutoa huduma za ushauri na uhamasisho kwa watu wengine wa Watu wenye Mahitaji Maalum kuhusu haki na wajibu wao.

Kikundi cha wanawake katika Ofua II kimenunua mashine saba za kushona nguo na vifaa vingine vya kuwafunza wanawake wengine. Kufikia hapa, kikundi hiki kimetoa mafunzo kwa wanawake 16 ya kushona nguo na wanawake 5 kwa utengenezaji viatu. Mafunzo yanayoendelea yanajumuisha kushona nguo (kwa wanawake 10) na kutengeneza shanga/kufuma/kusuka (kwa wanawake 56). Wanachama wa kikundi hutoa kiasi cha takriban Ugx 20,000 kama ada ya uanachama, Ugx 500 za usajili na Ugx 1,000 kila juma. Kikundi hiki huamua kwa pamoja jinsi ya kutumia pesa hizi.

\section{Washiriki:}

Baraza la Wakimbizi nchini Denmark

Ofisi ya Waziri Mkuu

\section{Kwa habari zaidi, wasiliana na:}

Jael Chunge: chunge@unhcr.org

Kalsum ljoru: ijoru@unhcr.org

\section{Mfano wa Kisa kutoka Uganda: Mkakati wa Kukuza Amani ya Kijamii}

ufuatia tukio la mauaji ya kijana wa uraia wa Uganda lililotekelezwa na wakimbizi katika makazi ya Kambi ya wakimbizi ya Rhino (Eneo la Eden) uhusiano baina ya jamii za wakimbizi na wenyeji ulikwazika na kuhatarishwa. Hivyo, kulihitajika kushughulikiwa na kutatua hali ile na kurudisha amani iliyokuwepo awali kati ya jamii hizi mbili kabla ya kutokea kwa tukio lile la kuhuzunisha. Mkakati wa Kukuza Amani ya Kijamii ulianzishwa kutokana na hali hii.

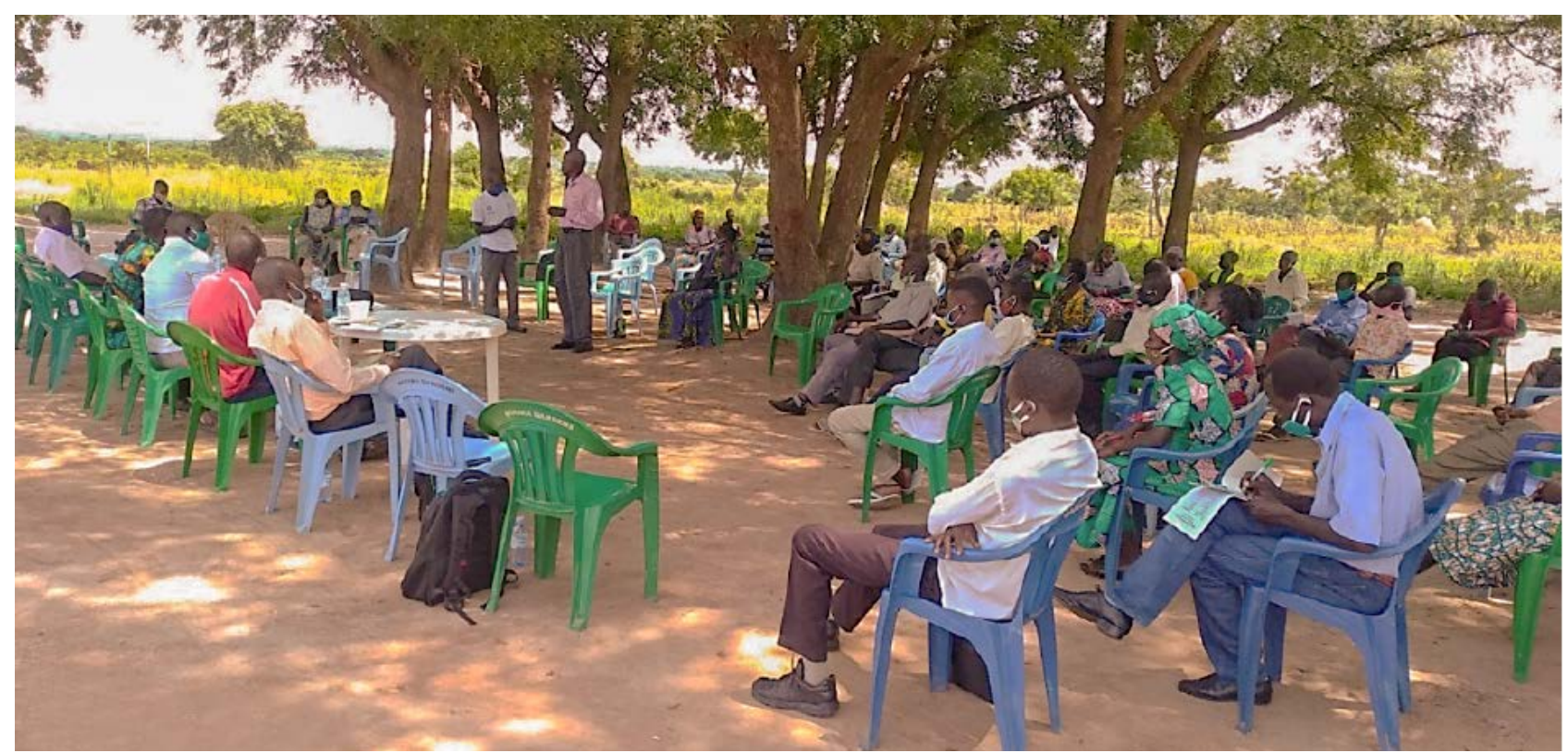

a Eden na viogozi wa jamii ya wenyeji. Mazungumzo manne yalifanyika - mawili yakifanyika na wenyeji na mawili yakifanyika na jamii ya wakimbizi. Kufikia kuanza kwa mazungumzo haya, washukiwa walikuwa wamekamatwa. Wakati wa mazungumzo, jamaa ya aliyekuwa amefariki walikemea uhalifu huo huku wakikubali kuwa kushutumiwa kwa wakimbizi wote kutakuwa si sawa na kuwaonya raia dhidi ya kutaka kulipiza kisasi.

Mazungumzo aya yalitoa nafasi ya wanajamii ya wakimbizi kuonyesha majuto na kuomba msamaha kutoka kwa wanajamii wa eneo lile waliokuwa wamekosewa. Wanajamii ya wakimbizi walijilaumu kutokana na tukio lile, wakiwaeleza wenyeji kuwa walikuwa wamekimbia kutoka kwa nchi yao ili kujiokoa; kuwa kutekeleza mauaji ni kinyume na sheria; na kuwa sio wakimbizi wote walikuwa wamehusika na uhalifu ule.

Jambo hili lilisuluhishwa na kukawa na makubaliano kuwa wakimbizi na wanajamii wa eneo lile wanapaswa kuishi kwa pamoja na kuendelea kutumia raslimali iliyopo kwa pamoja. Wakimbizi waliokuwa wamenunua au kukodisha ardhi walishauriwa kuendelea na ukulima, kukusanya kuni, kukusanya bidhaa za ujenzi na kadhalika. Jamii zote mbili zilikubali kusahau tukio la kusikitisha lililokuwa limetokea na kufungua ukurasa mpya kwa pamoja. 


\section{Washiriki:}

Mfumo wa Jamii wa Ukuzaji wa Teknolojia

Baraza la Wakimbizi nchini Denmark

Shirika la Development Literacy Partner International

Baraza ya Wakimbizi ya Norway

Ofisi ya Waziri Mkuu

\section{Kwa habari zaidi, wasiliana na:}

Jael Chunge: chunge@unhcr.org

Ejoyi Geoffrey Bakole: ejoyi@unhcr.org

\section{Desturi Zinazojitokeza kutoka Uganda: Mkakati wa baiskeli ya magurudumu matatu}

W

atu wenye Mahitaji Maalum katika Makazi ya Wakimbizi ya Bidibidi wanaendelea kupata changamoto za kupata mgawo wa chakula kutokana na umbali wa maeneo ua ugavi wa chakula (FDP) katika makazi yale. Wengine wao hutembea hadi umbali wa kilomita tano ili kufikia vituo vya ugavi na hulazimika kuuza sehemu ya mgawo ili kupanga jinsi ya kuchukuliwa na kufikishiwa mgawo huo. Katika juhudi za kupunguza mzigo wa usafirishaji kwa Watu wenye Mahitaji Maalum, UNHCR ilianzisha Mkakati wa baiskeli ya magurudumu matatu ili kuhakikisha ufikiaji salama na bora wa msaada wa chakula kwa hasa watu walio hatarini kati ya Watu wenye Mahitaji Maalum kupitia mbinu rahisi na endelevu ya usafiri.

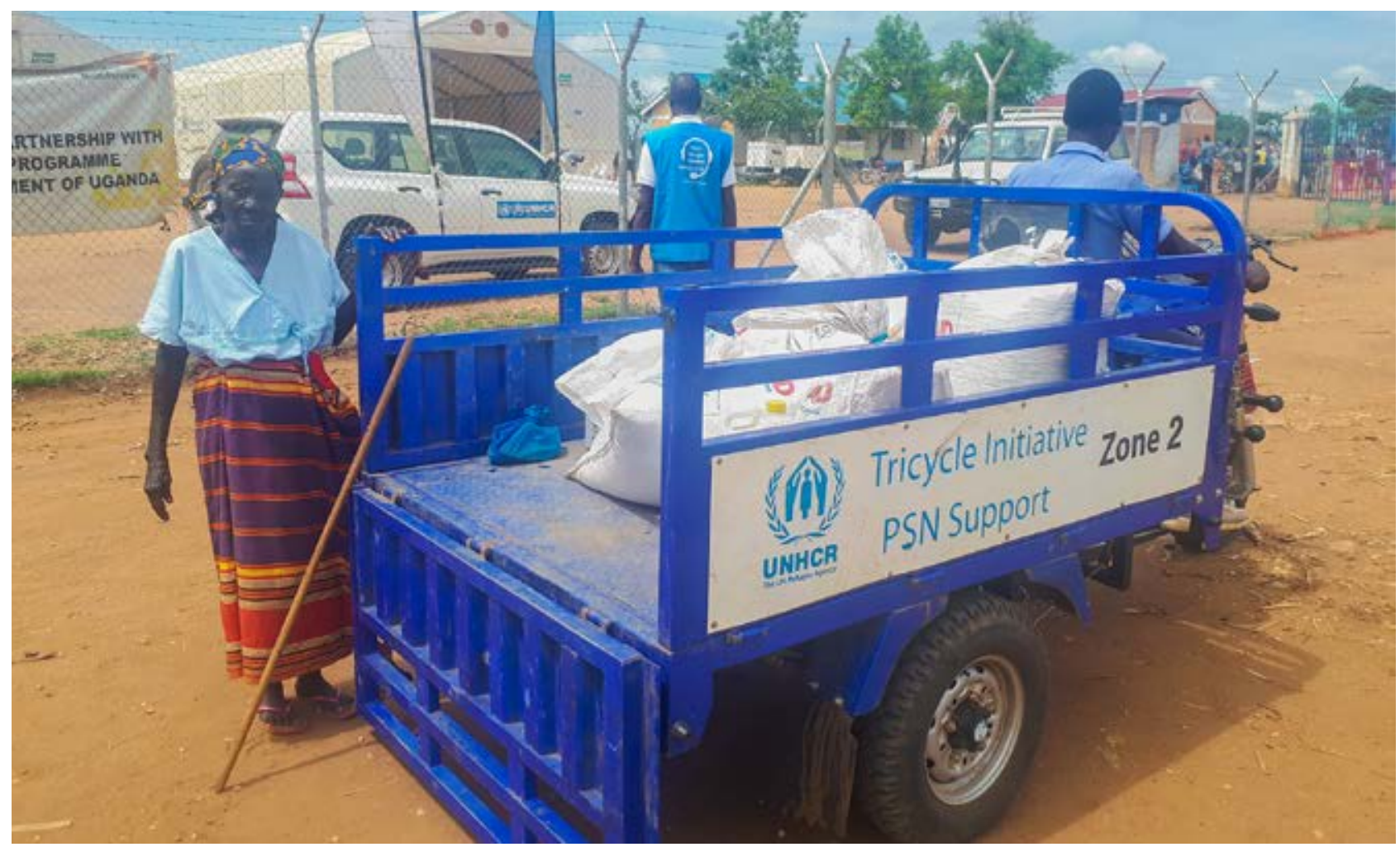

Mradi huu una malengo mawili: 1) kuwezesha huduma ya usafiri usiolipishwa kwa njia salama kwa hasa familia zilizo hatarini kutoka kwa FDPs hadi manyumbani mwao katika makazi haya, na 2) kusaidia fursa za shughuli za kujikimu maisha kwa Vyama vya Kijijini vya Kuweka Akiba na kutoa Mikopo (VSLAs) katika siku zisizo za ugavi wa chakula.

Ili kutimiza malengo haya, UNHCR ilitenga baiskeli 20 za magurudumu matatu za injini kwa Makazi ya Wakimbizi ya Bidibidi. Baiskeli hizi zilisambazwa kwa kila eneo kwa VSLAs vilivyochaguliwa kulingana na mahitaji ya kila eneo. Utengaji huu ulifanyika kwa kuzingatia ukubwa na topolojia ya eneo pamoja na umbali kutoka kwa FDPs na idadi ya hasa watu walio hatarini wanaohitaji kusafirishwa katika kila eneo. Washiriki wenza walisaidia kutambua watu hawa kupitia mtazamo wa kijamii kwa kuhusisha muundo ya jamii (katibu wa Baraza ya Maslahi ya Wakimbizi wa Watu wenye Mahitaji Maalum). VSLAs pia walichaguliwa kati ya makundi zaidi ya 1,000 kupitia mtazamo baina ya mashirika unaojumuisha washiriki wote wa ulinzi na wanaoshughulikia hali ya maisha pamoja na Ofisi ya Waziri Mkuu (OPM). 


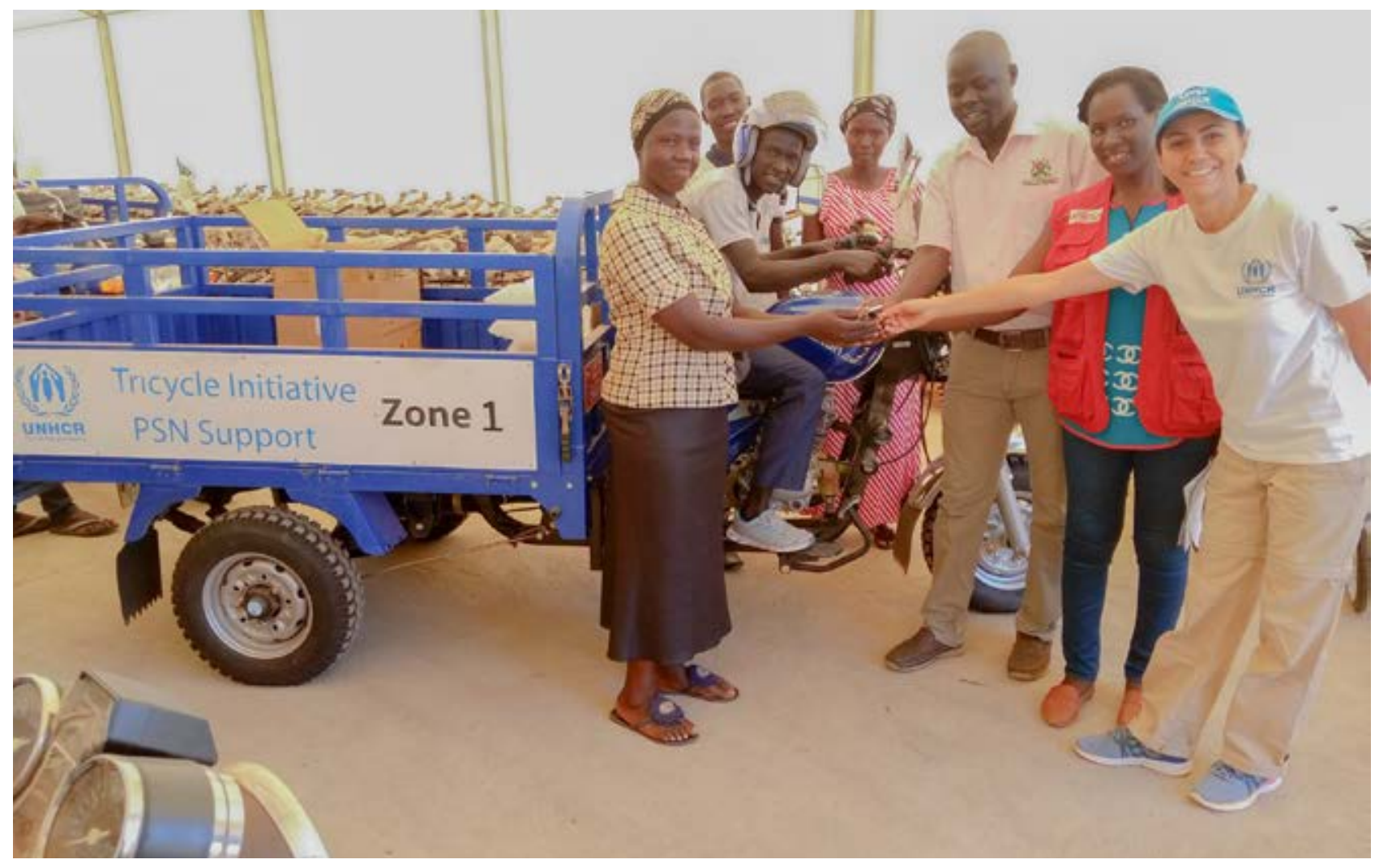

Makundi haya yalichaguliwa kwa kutathmini uzoefu na kiwango na ushahidi wa shughuli za pesa za akiba na mikopo pamoja na kupendelea kwa kikundi kuwahudumia Watu wenye Mahitaji Maalum, jambo lililotambuliwa kwa kuwahoji wanachama wa kikundi hicho.Baadhi ya makundi huwa na jinsia maalum huku mengine yakiwa na jinsia mseto, kulingana na muundo wa kibiashara wa VSLA, ila mchanganyiko wa waume na wake walikuwa baadhi ya waliochaguliwa katika mkakati huu.

VSLAs vinavyochaguliwa vilipewa mafunzo ya jinsi ya kuendesha baiskeli ya magurudumu matatu na watu wawili katika kila kikundi kupewa kibali cha kuendesha. Mafunzo na vibali vya kuendesha vilishughulikiwa na Shirika la Dan Church Aid. VSLAs vilisaidiwa kupata mafuta na huduma ndogo na ukarabati kwa kipindi cha miezi mitatu ya kwanza kwa kuwapa muda wa kuweza kujitegemea. MoU uliafikiwa kati ya kila VSLA, DCA, UNHCR, OPM na IRC uliokuwa ukitaja lengo la mkakati huu na majukumu ya vyama vyote.

Baiskeli 20 za magurudumu matatu zilipatiwa makundi katika maeneo tofauti katika Makazi ya Bidibidi. Baiskeli hizi zinafanya kazi vyema kwa kusaidia hasa watu walio hatarini wakati wa kusambaziwa chakula katika maeneo yao katika kusafirisha vyakula bila kulipishwa hadi kwenye manyumba yao kwa wastani wa siku tano kwa kila msambao. Muda huo mwingine wa mwezi, baiskeli hizi hufanya shughuli zingine za kuleta mapato kwa kuzingatia sheria na masharti walizoafikiana katika MOU. Mkakati huu umekuwa na athari chanya katika kuleta mapato kwa VSLAs.

\section{Matokeo}

Tamko lifuatalo kutoka kwa mwanachama wa VSLA linajumlisha matokeo yaliyoletwa na mkakati huu kufikia wakati huu: 'Tulipoanza kutumia baiskeli hizi, katika mikutano yetu ya kila juma, wanachama walikuwa wakiweka akiba ya UGX 3,000. Tunapanga kuongeza kiwango chetu cha akiba hadi UGX 4,000 katika mzunguko wetu wa mwaka ujao ambao utaanza Oktoba 2020, miezi tisa baada ya kuanza kwa Mkakati wa baiskeli ya magurudumu matatu. Licha ya hali ya ungonjwa wa corona na vizuizi vya usafiri, bado tunaweza kupata kazi tofauti katika makazi na mradi uliongeza kiasi cha mapato ya VSLA. Hapo awali, kiasi cha juu zaidi cha mkopo kilikuwa ni UGX 500,000. Hata hivyo kwa sasa tunaweza kutoa mikopo ya hadi UGX 1,000,000. Hii itasaidia wanachama kufikiria kuhusu miradi mikubwa na hivyo kuongeza uwezo wao kwa kiasi kikubwa'

\section{Washiriki:}

Shirika la Dan Church Aid

Shirika la International Rescue Committee Ofisi ya Waziri Mkuu

\section{Kwa habari zaidi, wasiliana na:}

Elham Baghdadi: baghdad@unhcr.org

vincent Bukenya: bukenyav@unhcr.org 


\section{Mfano wa Kisa kutoka nchi ya Sudan Kusini: Mfumo wa Ulinzi wa Kijamii kwa IDPs}

$\mathrm{M}$

kurupuko wa mgogoro nchini Sudan Kusini ulisababisha watu wengi kukimbilia maeneo ya mjini. Licha ya kuwepo kwa makubaliano ya amani yaliyoafikiwa, wakimbizi hawa wandani bado wanaogopa. Wakimbizi wengi wa ndani (IDPs) wanahofia maisha yao na hawapendelei kurejea makwao. IDPs wengi waliachwa hatarini baada ya manyumba yao kuharibiwa na shughuli zao za kupata mapato kuharibiwa kutokana na vita.

Ili kuwasaidia IDPs, Mfumo wa Ulinzi wa Kijamii ulianzishwa kama muundo muhimu wa ulinzi utakaokuza ushiriki wa jamii katika ukadiriaji wa shughuliza kibinadamu au usambazaji, utambuzi wa nyumba zilizo hatarini katika jamii za IDP na kugawana habari katika maeneo 9 ya IDP katika mji wa Yei. Muundo mpya wa kijamii ulianzishwa huku miundo iliyokuwa ikiimarishwa kama mkakati wa Ulinzi. Mtazamo huu ulikusudiwa kuhakikisha umiliki wa jamii na uendelevu wa mkakati. Mfumo wa Ulinzi wa Kijamii unatoa jukwaa kwa wanajamii kusaidiana mahitaji ya kila siku na kukuza na kusambaza desturi bora zaidi, miongozo na mikakati ili kutumika na wanachama wote.

Chini ya mfumo huu, mafunzo endelevu ya kazi kwa miundo ya jamii yalifanyika ili kuboresha stadi na msaada unaotolewa kwa IDPs walio hatarini na wenyeji. Ili kuongeza matumizi ya raslimali zilizoko na kuepuka kulenga kujirudia kwa juhudi, shirika la United Methodist Committee on Relief umeungana na mashirika mengine ya kibinadamu wakati wote katika mkakati huu. Shughuli zinazopendekezwa za mradi zilifanyika katika maeneo 9 ya IDPs kwa kusaidiwa na Serikali ya taifa. Miundo iliyopo ilichangia pakubwa katika kuhamasisha na kufahamisha, kutambua watakaonufaika, kuripoti ukiukaji wa ulinzi na kuitikia wahasiriwa wanaotafuta msaada na haki kwa ajili ya dhulma walizofanyiwa.

Mfumo wa Kijamii umesaidia kwa njia hii:

- Mfumo unaofanya kazi wa kufuatilia ulinzi wa kijamii, na kusababisha ongezeko katika ufahamu kuhusu hatari za SGBV, njia bayana za kuripoti na taratibu za haraka na zinazoheshimu usimamizi wa visa kwa manusura wa SGBV

- Mbinu bora na inayofanya kazi ya ukusanyaji data yenye uhakiki na inayoheshimu usiri.

- Makundi ya msaada yanayofanya kazi yaliyoanzishwa ya Watu wenye Mahitaji Maalum ili kuhakikisha kujilinda na kujisimamia kwa jamii

Washiriki:

Shirika la United Methodist Committee on Relief

Shirika la Mission to Alleviate Suffering in South Sudan

Kwa habari zaidi, wasiliana na:

Wamutshilani Nkala: nkalaw@unhcr.or 
Maadili. ROVs hupewa jukumu la kutambua visa vinne kila mwezi vya Watu wenye Mahitaji Maalum na ambao wanahitaji umakini wa UNHCR au washirika wake. Visa vilivyotambuliwa hufuatiliwa, na maoni kuhusu hatua zinazochukuliwa hupewa kwa ROVs.

\section{Matokeo}

Matokeo yanapimwa kulingana na ripoti za kila mwezi za Watu Husika walioshughulikiwa, waliopewa rufaa kwa watoaji huduma, idadi ya ufuatiliaji iliyofanyika na mahitaji yaliyotoshelezwa. Maoni kutoka kwa Watu Husika na wasimamizi yanarekodiwa na kuhifadhiwa katika faili ya kila ROV ikiwemo katika hifadhi ya data ya UNHCR inayofahamika kama UNHCR ProGres*.

\section{Washiriki:}

Shirika la Maslahi ya Wakimbizi na Wanaorudi

Tume ya Ukuzaji wa Msaada Baina ya Makanisa

Shirika la Jesuit Refugee Service

Kwa habari zaidi, wasiliana na:

Nunu Aliyi: aliyi@unhcr.org

\section{Mfano wa Kisa kutoka Kenya: Usawa wa Kijinsia kulingana na mpango wa WASH}

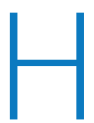
uduma za mpango wa WASH katika kambi ya wakimbizi za Kakuma na makaazi ya Kalobeyei zimepangwa haswa kutolewa kwa Watu wote Husika wakiwemo wanawake, wanaume, wasichana na wavulana - kwa vipimo venye usawa. Msisitizo maalum unawekwa kwa wanawake na wasichana kwa maana kuna uwezekano mkubwa wao kubaki nyuma na kutowakilishwa katika mikabala ya ushiriki wa kawaida wa kijamii.

Katika ngazi ya kambi, wanaonufaika kutokana na msaada wa mpango wa WASH hutambuliwa kupitia timu zenye kazi tofauti kama UNHCR, Tume ya Masuala ya Wakimbizi, Washirika wanaotekeleza huduma za Kiafya na washirika wengine. Familia zinazoongozwa na wanawake zinapewa kipaumbele. Katika kiwango cha vijisehemu, Kamati za mpango wa WASH (miundo ya wawakilishi wa kijamii wa mpango wa WASH) wanafunzwa kutambua na kuratibu masuala ya mpango wa WASH katika maeneo husika. Baraza ya Wakimbizi ya Norway limehakikisha uwakilishi wa aina mbali mbali wa uongozi baina ya kamati za WASH, ikiwemo uwiano wa 50:50 kati ya wawakilishi wanaume na wanawake. Wagombea wa kike hupewa kipaumbele katika kuajiriwa kama wafanyakazi wa WASH wa kutoa huduma katika jamii pia.

\section{Washiriki:}

Baraza ya Wakimbizi ya Norway

Kwa habari zaidi, wasiliana na:

Moe Miyahara: miyahara@unhcr.org

Oscar Nabiswa: nabiswa@unhcr.org

John Wagacha Burton: burtonj@unhcr.org

\section{Mfano wa Kisa kutoka Uganda: Mpango wa Kazi/Jedwali la Viashiria vya Umri, Jinsia, na Uanuwai}

$<$ utumia mkabala wa Umri, Jinsia na Uanuwai (AGD) katika kazi yetu ni muhimu ili kuhakikisha kuwa huduma zetu ni salama na rahisi kupatikana na kila mtu na zinapeanwa bila ubaguzi. Mkakati huu unatuhitaji tuzingatie mahitaji tofauti na maoni ya wanawake, wanaume, wasichana na wavulana kutoka asili tofauti pamoja na Watu wanaoishi na Ulemavu, watu wenye mielekeo tofauti ya kimapenzi na utambulisho wa kijinsia na kikabila, vikundi vidogo vya kidini na kilugha ama makundi asili katika mchakato wa kutathmini, kupanga, kutekeleza na kufuatilia.

Ili kuhakikisha kuwa washirika na sekta zote za UNHCR zinatumia Mtazamo wa AGD katika kazi zao, timu zinazohusika na Ulinzi wa Jamii (CBP) na za Mipango zilibuni mipangilio ya kazi na utaratibu wa kuzingatia AGD.Mpango wa Kazi wa sasa wa AGD una msingi wa Mpango wa Kazi wa AGD wa kitaifa uliotengenezwa katika mwaka wa 2019. Mpango wa Hatua wa kitaifa wa AGD uliimarisha mipango ya hatua ya mashinani iliyoundwa baada ya warsha ya kina katika mwaka wa 2018 na ina orodha ya changamoto, shughuli za mashinani na shughuli za kiwango cha kitaifa ili kutekeleza kwa ufanisi sera ya AGD ya UNHCR. Mpango huu wa hatua uliendelezwa zaidi kupitia maushauriano na uongozi wa sekta mjini Kampala, 
ulinzi wa mashinani kwa wafanyakazi na washirika. Mpango wa Kazi wa AGD uliundwa katika Mpango wa Hatua wa AGD ila unafanana na jedwali la viashiria, kuleta viashiria vya mapato na matokeo, misingi na malengo. Hii itatusaidia kufuatilia kwa ufanisi kutekelezwa kwa shughuli katika mpango wa kazi. Mpangilio wa kazi/jedwali utakamilishwa kupitia mashauriano zaidi na uongozi wa sekta (ndani na nje ya UNHCR) na wafanyikazi wenza wa mashinani. Hii imekuwa juhudi ya pamoja kati ya CBP na walioshiriki katika mpangilio.

Matokeo ya mpangilio wa kazi wa AGD wa 2020-2021 bado hayajatathminiwa. Hata hivyo, inatarajiwa kuwa na athari katika njia ambayo huduma zinatolewa nchini Uganda, na jinsi ya kutekeleza sera ya AGD ya UNHCR. Matumizi yanayofaa ya mtazamo wa AGD katika kazi ya mshirika ingeongeza usalama na upatikanaji wa huduma kwa wote na kuwezesha makundi yaliotengwa na yalionyimwa haki.

Mchakato wa kufanya kazi na washiriki na wafanyakazi wa UNHCR umeongezea kwa kiwango kikubwa ufahamu wa sera hio. Ushirikiano kati ya Ulinzi na Mpangilio umekuwa mzuri sana. Kuhusika kwa Afisa Mkuu wa Upangiliaji umeufanya mpango huo kuwa mkakati wa UNHCR, bali si mkakati wa Ulinzi.

Washiriki: mbalimbali

Kwa habari zaidi, wasiliana na:

Sam Mosallai: mosallai@unhcr.org

Yoko Iwasa: iwasa@unhcr.org

Clare Askew: askew@unhcr.org

\section{Mfano wa Kisa kutoka Uganda: Mipango ya Kazi ya Watekelezaji kulingana na vigezo vya Umri, Jinsia, na Uanuwai}

M kabala unaozingatia Umri, Jinsia na Uanuwai (AGD) unakuza mazingatio ya maana kwa hali halisi, maoni na mahitaji ya wanawake, wanaume, wasichana na wavulana wa asili tofauti katika mchakato wa kutathmini, kupanga, kutekeleza na kufuatilia. Makundi ya watu yenye mvuto ni Watu wanaoishi na Ulemavu, watu wenye mielekeo tofauti ya kimapenzi na utambulisho wa kijinsia na kikabila, kidini na makundi madogo ya kilugha ama makundi asili.

Kuendeleza matumizi ya mtazamo wa AGD katika kazi ya watekelezaji, UNHCR imefanya sera yake inayozingatia Umri, Jinsia na Uanuwai kuwa hitaji la lazima kwa washiriki wote watekelezaji. Timu ya Ulinzi Wa Kijamii, kwa msaada wa wenza katika mpangilio, imekuwa ikifanya kazi na washiriki wapya watekelezaji ili kujenga uwezo wao wa mtazamo wa AGD. Kabla ya shughuli hii ya kujenga uwezo, violezo vya Makubaliano ya Ushirikiano katika Mradi (PPA) ya UNHCR vilijuimuisha marejeleo machache ya Umri,Jinsia na Uanuwai, bila kutaja matarajio ambayo washiriki wanafaa kuzingatia sera ya AGD ya UNHCR. Hivyo, washiriki hawakufahamu kuwepo kwa sera hii.

Kurekebisha hali hii, timu ya CBP ilifanya kazi kwa ukaribu na wenzao katika mchakato wa kuchagua washiriki wa 2020. Wahusika wote wa CBP walihudhuria mikutano ya utangulizi na washirika wapya, walitoa mawasilisho kuhusu sera ya AGD, walijulisha washiriki wajibu wao wa kuzingatia sera na kuwapa misaada ya Umri, Jinsia na Uanuwai. Zaidi ya hayo, marejeleo ya kina ya mtazamo wa AGD yalijumuishwa katika PPA zote na barua za maombi na ikakubaliwa kuwa washiriki wote wangeendeleza mipangilio ya kikazi ya kibinafsi na yenye umaalum wa kisekta. Kwa kuongeza, kipindi cha mafunzo cha nusu siku kilifanywa ambapo washiriki walihamashishwa zaidi kuhusu sera ya AGD pamoja na Kamati Maalum inayohusika na masuala ya Jinsia Baina ya Mashirika yenye kuzingatia Umri. Washiriki pia walipata mwongozo kuhusu kuendeleza mipangilio ya kikazi inayozingatia AGD ambayo ni ya washiriki wa kisekta ya mwaka uliofuata, ili kuhakikisha Usawa wa Kijinsia (GE) na Uwajibikaji kwa Watu Walioathiriwa (AAP) katika shughuli zote za washiriki. Mipango hii ya kikazi inajumuisha shughuli, viashiria, watu husika na tarehe za kukamilisha. Maendeleo yanayofanywa katika kutekeleza mipango hii ya kazi yatafuatiliwa katikati ya mwaka na mwisho wa mwaka ili kupata hakiki ya utendakazi.

Athari ya kujumuisha AGD katika PPA za 2020, kuanzisha mahitaji ya lazima ya kuripoti, na kuendeleza mipangilio ya kikazi inayozingatia AGD ya washiriki wa sekta maalum zinatarajiwa kuathiri kutoa huduma katika makaazi ya wakimbizi nchini Uganda. Matumizi yanayofaa ya mkabala wa Umri, Jinsia na Uanuwai katika kazi ya mshirika huweza kuongeza usalama na kupata huduma kwa wote na kuwezesha makundi yaliotengwa na yaliyonyimwa haki. Mchakato wa kufanya kazi na washirika na wafanyakazi wa UNHCR umeongezea kwa kiwango kikubwa ufahamu wa sera hio.

Washiriki: mbalimbali

Kwa habari zaidi, wasiliana na:

Sam Mosallai: mosallai@unhcr.org

Yoko Iwasa: iwasa@unhcr.org 


\title{
Matokeo
}

Wakimbizi na wenyeji wanapata kinu cha kusaga kwa bei nafuu na kinachoweza kutegemewa. Eneo la kinu katika jamii ya wakimbizi hupunguza hatari za kiulinzi na kiusalama kwa wanawake, wasichana na vijana. Chama hiki cha Wanawake chenye kujitegemea kimezalisha faida, jambo ambalo limepelekea uamuzi wa chama kuongezea wanawake zaidi kutoka kwa jamii ya wakimbizi na kupanua mradi huu kwa kujitosa katika ulingo wa urembo na biashara ya mapishi katika kambi. Mpangilio wa upanuaji wa kibiashara unachunguzwa kwa sasa na Chama hiki cha Wanawake. Kinu hiki kimeleta nafasi za kazi ya malipo kwa zaidi ya Watu 4 Husika katika kambi. Wanawake wanaojihusisha na mradi huu wameendeleza maisha yao na wanachangia kifedha katika familia zao. Mradi huu umevutia wanawake zaidi wanaotaka kujiunga katika miradi kama hio ya kupata riziki.

Washiriki:

Shirika la Maslahi ya Wakimbizi na Wanaorudi

Kwa habari zaidi, wasiliana na:

Alisona Rajbanshi: rajbansh@unhcr.org

\section{Mfano wa Kisa kutoka Kenya: Mikakati ya Kijinsia ya mpango wa WASH kama Njia ya Kuzuia SGBV}

\begin{abstract}
Ii kuzuia wahalifu wa SGBV katika kambi za wakimbizi, vituo vya maji vimejengwa kwa makusudi katika nafasi wazi kwa jamii. Zaidi ya hayo, usambazaji wa maji unafanywa kuanzia saa 1 asubuhi hadi saa 11 jioni, wala sio usiku il kuhakikisha usalama kwa ujumla kwa wanajamii. Kupunguza mgogoro katika vituo vya maji (mahali penye mifereji), kamati ya mpango wa WASH na viongozi wa wakimbizi wamefanya kazi pamoja na wanajamii kuendeleza ratiba ya kuchota maji asubuhi, adhuhuri na jioni.
\end{abstract}

Isitoshe, vyoo vipya vya umma vimeundwa kwa ajili ya wakimbizi wapya, na vimewekewa vizuizi vya ndani ili kuhakikisha usiri. Wanajamii pia wameshauriwa kuandamana na watoto na wanawake msalani wakati wa usiku.

\section{Washiriki:}

Baraza ya Wakimbizi ya Norway

Kwa habari zaidi, wasiliana na:

Moe Miyahara: miyahara@unhcr.org

Oscar Nabiswa: nabiswa@unhcr.org

John Wagacha Burton: burtonj@unhcr.org

\section{Desturi Inayojitokeza kutoka Kenya: Nyumba Salama Zinazoendeshwa na Jamii}

$\bigcup$ NHCR na washirika wake wana wataalam ambao wamejitolea kutoka kwa jamii ya wakimbizi na ambao wamefungua wazi nyumba zao ili kutoa nafasi salama za manusura wa SGBV wanaohitaji mahali ya kukaa kwa muda na usaidizi huku wakisubiri tathmini ya mahitaji yao na masuluhisho. Wataalam wanaojitolea hupeana makao ndani ya nyumba zao na husaidia kuelekeza manusura kwa huduma wanazohitaji, kama vile huduma za afya. Hatua hii inahakikisha kuwa manusura ambao hawahitaji kuwa katika taasisi hizo kwa muda mrefu bado wanapata mahali salama katika jamii, na kuhakikisha kuwa uwezo wa kijamii unadumumishwa ili kutambua suluhisho na msaada kwa njia huru.

Manusura wa SGBV hupewa msaada wa vifaa, huduma ya kwanza ya kisaikolojia, kuelekezwa kwa huduma maalum katika misingi ya kidharura na ya kujitolea. Visa vyenye hatari ya kiwango cha chini na cha wastani pekee vinashughulikiwa katika nyumba salama za jamii baada ya tathmini ya usalama kufanywa kwa ruhusa ya aliyenusurika. Mkabala unaozingatia aliyenusurika unasisitizwa katika kuzingatia kuchukua manusura katika makao salama katika jamii. Maoni ya wanaojitolea ikilinganishwa na maelezo na mahitaji ya visa huzingatiwa pia ili kuhakikisha hatari imeepukwa kwa manusura na wanajamii waliojitolea. Mikutano ya uratibu ya mashirika mbalimbali ya mara kwa mara na makongamano ya dharura hufanywa ili kutathmini maendeleo ya manusura katika mchakato wote. 


\section{Matokeo}

Makao salama ya jamii yamepokewa kama sehemu za ziada na njia mbadala za nafasi salama za taasisi kwa kuwa huduma kama hizo huwa adimu na hukosekana. Mpango huu pia umetumika kutoa makao salama kwa wanaume na wavulana na watu wenye Mwelekeo tofauti ya Kimapenzi, Utambulisho wa Jinsia na Kujieleza - watu ambao hawajajengewa makao haya salama na kwa ambao kupata huduma hii huwa na changamoto.

Jamii imeweza yenyewe kukabiliana na visa visivyohitaji usalama wa kidharura na makao salama huku ikisubiria majadiliano na wataalam wa visa vya SGBV. Kubaini na kuripoti visa vya SGBV katika jamii vile vile imeboreshwa, huku waliojitolea wakiwa watu wa msingi ambao wanajamii wanaweza kujadiliana nao.

Washiriki: haihusiki

Kwa habari zaidi, wasiliana na:

Aisha Ngonze: ngonzea@unhcr.org

\section{Desturi Inayojitokeza kutoka Kenya: Watunzi katika Jamii - Makao Salama Mjini ya Muda ya Manusura wa SGBV}

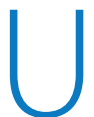
NHCR na Jumuiya ya Misaada ya Wahamiaji wa Kiebrania (HIAS), wamefanya kazi na wanajamii katika miundo ya wakimbizi iliyoko mjini ili kukuza idadi ya wanaojitolea kutoa makao yao kama sehemu salama na yasiyo ya kudumu kwa manusura wa SGBV. Katika eneo la Kayole mjini Nairobi, watoaji wa huduma/watunzi hupata angalau mafunzo mara mawili kila mwaka ili kuongeza uwezo wao wa kusaidia manusura wa SGBV. Wao pia hupata pesa kidogo ya kuwawezesha kuishi na manusura. Watoaji huduma katika jamii kutoka kwa washirika hufuatilia makao ya kifamilia na kuwapa msaada zaidi mtoa huduma na manusura.

Kamati za Jamii huhamasishwa kuhusu mpango wa kutoa huduma katika jamii na hufanya kazi kama jukwaa la kutambua na kuhakiki watoaji huduma katika jamii kabla ya kuandikishwa katika mpangilio huu. Pale ambapo hakuna mtoaji huduma wa kumchukua manusura, miundo nyingine katika jamii (kama vile makanisa) hutumika kama mahali salama kwa manusura.

\section{Matokeo}

Jambo hili ni suluhisho katika jamii ambayo husisitiza dhana kuwa jamii inaweza ikasaidia manusura SGBV bila kurejelewa kwa washiriki wa kawaida ambako kunaweza kusifanye kazi katika wakati mgumu (kama usiku). Hatua hii imepelekea manusura kupata huduma ya haraka ikiwemo kuhakikisha kuwa wamepata makao salama, vituo vya afya na vituo vya polisi pamoja na ushauri wa mara moja.

Watoaji huduma katika jamii wamejenga mahusiano mazuri ya kikazi na serikali hususan polisi na maafisa wa serikali ya kaunti kutokana na utangamano na washikadau hao katika kazi za kuwasaidia manusura manyumbani mwao. Wanajamii wengine ambao si watoaji huduma wa kijamii pia hujitolea mara kwa mara kuchukua manusura wa SGBV.

\section{Washiriki:}

Jamii ya Msaada kwa Wahamaji ya Kiebrania

Sekretariati ya Masuala ya Wakimbizi

Kikundi cha Utendakazi cha Dhulma ya Kijinsia

Washirika wengine wa SGBV

Kwa habari zaidi, wasiliana na:

Jackson Karugu: karugu@unhcr.org

Daniel Mureithi: mureithi@unhcr.org 


\section{Desturi Zinazojitokeza kutoka Rwanda: Mpango wa Kushughulikia Ujauzito miongoni mwa Vijana na visa vya kuacha Shule}

jauzito miongoni mwa vijana ni changamoto ambayo imekuwepo kwa muda mrefu katika kambi ya Kiziba, ikiongeza
hatari ya kuacha shule kwa wasichana. Kutoka Mwezi wa Machi hadi Agosti 2020, wanafunzi 22 wa kike walitambulika
kuwa wajawazito na walikuwa katika hatari ya kuacha shule.

Sababu kadhaa zinazoendeleza kuacha shule kwa wanafunzi wajawazito katika kambi ni pamoja na ukosefu wa msaada kutoka kwa familia na jamii wa kuwarudisha wasichana hawa shuleni, woga wa kutengwa na walimu na wenzao shuleni na ukosefu wa muundo wa kupunguza hatari ya kuacha shule pale msichana anapokuwa mjamzito. Mpango wa hivi sasa umelenga: kutathmini changamoto ya kuacha shule kupitia mpangilio fulani; kuelewa hatari za kuacha shule kwa vijana wa kike kutokana na ujauzito kupitia kuzungumza na kikundi lengwa; kukuza msaada wa kifamilia na kijamii kwa wasichana wajawazito ili kuwawezesha kurudi shuleni ikiwa kama mpango wa kuzuia SGBV; na hatimaye kuwawezesha kurudi katika mfumo wa kimasomo.

Mpango huu ulijumuisha vipengele vifutavyo:

- Kutafuta kwa kina watoto walioacha shule, hii ikiwezeshwa na walimu wa wakimbizi na mshirika wa UNHCR wa kutoa mafunzo, mpango ambao uliwezesha watoto 185 wa umri kati ya 3-19 kutambuliwa kuwa waliacha shule kutokana na sababu tofauti.

- Majadiliano na makundi ya wasichana, wavulana, wazazi na walimu ili kuelewa vyema hatari ya kuacha shule miongoni mwa wasichana wajawazito. Wasichana walieleza walichohisi kuhusu maisha yao walipogundua kuwa ni wajawazito. Wazazi wao waliona aibu sana kuwaruhusu kurudi shuleni na wasichana walipatwa na wasiwasi wa kukejeliwa shuleni.

- Kuanzishwa kwa muundo wa kijamii ulio na walimu wa wakimbizi na CPCM wa kupanga ziara za kinyumbani na kutoa ushauri kwa familia za wasichana wajawazito waliotambulika na wakuu wa mashule. Muundo huu wa kijamii ulianzishwa kwa ushirikiano na vitengo vya Elimu na Ulinzi wa Watoto pamoja na timu ya CBP ya Karongi.

- Kutekeleza mafunzo ya walimu na CPCMs (kukiongozwa na washirika wa SGBV na washiriki wa kiafya) ili kuimarisha ufahamu na mawasiliano hivyo kuweza kupunguza hatari.

\section{Matokeo}

Kwa juhudi endelevu, katika mwaka wa kuanzisha hatua hii, wasichana 15 walioacha shule kutokana na ujauzito walirudi shuleni na baadhi yao kufuzu vyema katika mitihani ya kitaifa.

Washiriki:

Shirika la Kimataifa la World Vision

Walimu walio wakimbizi

Kwa habari zaidi, wasiliana na:

Ruizhi Zhu:zhur@unhcr.org; rwaki@unhcr.org

Speciose Buwamaria: buwamari@unhcr.org

\section{Desturi ya Kutia moyo kutoka nchi ya Rwanda: Kutambua Manusura na Kuitikia kupitia Ukaguzi}

japokuwa SGBV ni tatizo la kawaida katika kambi za wakimbizi, kutoripoti ni changamoto ambayo inawazuia manusura kupata hatua wanazohitaji kutoka sekta tofauti. Ili kuendeleza utambulisho wa manusura wa SGBV na kuwaunganisha na huduma katika njia rahisi, UNHCR, katika nchi ya Rwanda ilichukua njia ya kurejelea ushahidi wa SGBV iliyoanzishwa na Tume ya Idadi ya Watu na Hospitali Kuu ya Kenyatta, Kenya na ikachambuliwa na kambi za wakimbizi za Uganda kwa ushirikiano na UNHCR.

Mkakati huu unahusisha vipengele vifuatavyo:

- Mafunzo kwa Watoaji Huduma na Vipindi vya Kuweka Taratibu: UNHCR na Tume ya Idadi ya Watu iliwafunza wahudumu wa afya kuhusu kuchunguza SGBV na kwa pamoja wakaunda kifaa cha kufanya uchunguzi ya SGBV ikiwemo (ya kimwili, kiakili na kimapenzi) na kubakwa na mpenzi au mtu ambaye si mpenzi. Mafunzo 


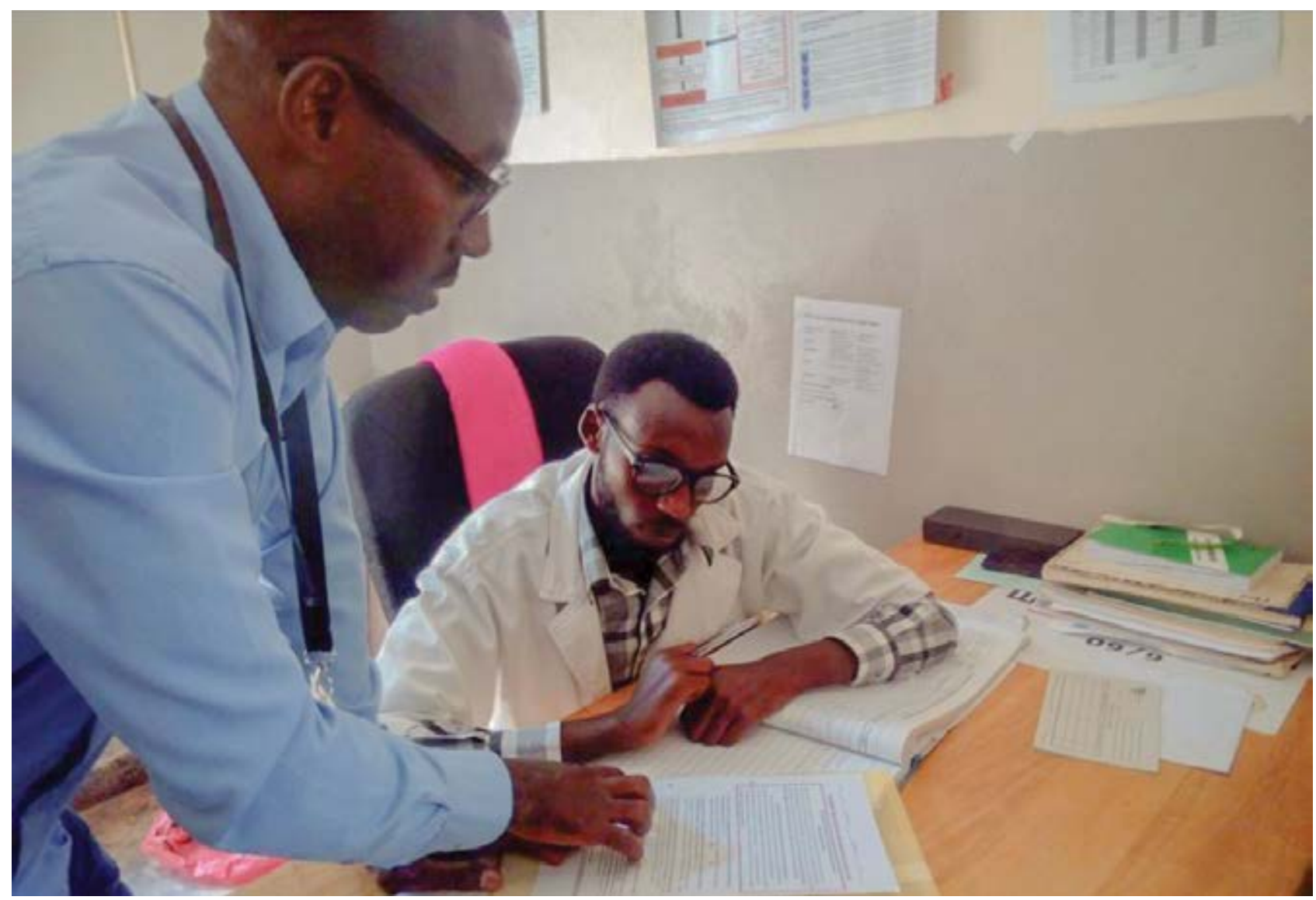

haya pia yaligusia kuhusu dhana ya SGBV, ufafanuzi wa maadili, maadili ya uchunguzi, mikabala ya manusura, kuelekezwa kwa msaada na kuhifadhi data. Walioshiriki mafunzo ni watoaji huduma kutoka kambi 5 za Rwanda, waliowakilisha idara/kliniki ambapo usiri wa vitu vinavyoonekana na kusemwa ungehakikishwa (haswa Idara za Wagonjwa wanaotibiwa na kurudi nyumbani, Magonjwa yasiyo ya kuambukiza, Wagonjwa waliolazwa, Huduma ya kujifungua, Huduma ya Kuzalisha, Virusi vya Ukosefu wa Kinga Mwilini na Lishe). Kipindi hiki kilitumika kama jukwaa la kufanya kazi kwa ushirikiano na watoa huduma ili kuchukulia mchakato mzima wa kuchunguza ukweli wa vituo vya kiafya.

- Uchunguzi na Kutoa rufaamaalum Wahudumu wa kiafya waliofunzwa (wauguzi, wataalamu wa lishe, washauri wa kijamii na wakunga) walitumia kifaa hiki cha uchunguzi katika mkutadha wa usiri, ushauri wa moja kwa moja na wateja wote wa kike kuanzia umri wa miaka 14 kuenda juu waliokuwa wakitafuta huduma za kiafya katika idara/kliniki husika. Wateja waliopatikana kuwa walipitia SGBV walielekezwa (kwa idhini yao) kwa vituo vinavyotoa huduma kwa waathiriwa wa jambo hili vilivyo katika vituo vyote vya kiafya. Ili kuimarisha kutoa rufaa, simu zilipigwa kati ya watoaji huduma pale ambapo manusura wa SGBV hawangepelekwa katika vituo

- Kukusanya data: Kliniki husika zilitumia violezo viwili vya kukusanya na kuripoti data kila mwezi ili kunasa idadi ya: wateja waliochunguzwa, manusura waliotambuliwa, manusura waliopewa marejeleo katika huduma ya umakini na manusura wanaopata huduma katika vituo vya SGBV. Visa vya SGBV kulingana na aina, uchunguzi, changamoto na mahitaji pia hunaswa na data hii kutengwa kwa wanawake na wasichana ili kuwezesha mpangilio.

Uchunguzi wa SGBV katika kambi za Rwanda ulianza katika mwaka wa 2017 katika kambi za Gihembe na Nyabihenke na katika mwezi wa Julai katika mwaka wa 2019 katika kambi za Mahama, Kigeme na Mugombwa na imeendelea tangu. Wahudumu wa afya wanaofanya kazi katika Kambi hizi wanafunzwa kuchunguza mteja wa kike ili kutambua manusura wengi haraka iwezekanavyo.

\section{Matokeo}

Katika mwaka wa 2017, vituo vya afya vilivyoshirikiswa katika kambi 2 nchini Rwanda vilipata ongezeko la asilimia 300 ya kiasi cha manusura waliotambuliwa na kuunganishwa na utunzi wa kina wa SGBV katika miezi 2 baada ya kuanzishwa kwa mkakati wa kukagua.

Katika mwaka wa 2019, katika kambi 5 zilizoshirikishwa nchini Rwanda, watu 326 walitambuliwa kuwa waathiriwa wa SGBV kupitia ukaguzi na kati yao 276 walipewa rufaa kwa huduma za kuitikia SGBV.

Katika mwaka wa 2019, vituo vya afya vilivyoshirikiswa katika kambi za wakimbizi katika jumla ya nchi 4 (ikijumuisha 
Rwanda) vilipata ongezeko la asilimia 129 ya kiasi cha manusura waliotambuliwa na kuunganishwa na utunzi wa kina wa SGBV katika miezi 6 ya kuanzishwa kwa mkakati wa kukagua.

Katika kambi 5 za nchi ya Rwanda ambapo ukaguzi wa SGBV unafanyika, mashirika ya kuitikia SGBV huripoti ongezeko la ghafla katika visa vinavyopokewa, jambo ambalo linafikiriwa kuhusiana na ongezeko la ghafla la utambuzi wa waathiriwa wa SGBV na kuweza kufikia utunzi. Data bora inayokusanywa kutoka kwa wateja na wanaotoa uduma katika kituo cha afya inaonyesha kuwa wanawake na wasichana wanafahamu kuwa wanaweza kuripoti matukio ya SGBV kwa kituo cha afya pamoja na mashirika ya kuitikia SGBV. Hii imesababisha kuongezeka kwa idadi ya visa vinavyotambuliwa vya SGBV.

Kuongeza, nchini Rwanda, hatua hii imetekelezwa kwa fanaka na UNHCR na washiriki wake watekelezaji Sudan Kusini na Zambia

Kwa pamoja na Baraza ya Idadi ya Watu, UNHCR imefanya mikutano ya kwanza na washirika wanaohusika na elimu, wilaya, walimu na wanafunzi, ili kutayarisha kuanzishwa kwa mkakati wa ukaguzi wa dhulma ya kimapenzi kwa watoto kwenye shule katika kambi za Mahama, Mugombwa na Kigeme. Mkakati huu, ambao pia ulianzishwa na kutathminiwa nchini Kenya na Baraza ya Idadi ya Watu na Hospitali ya Kitaifa ya Kenyatta, haujatekelezwa kwa sababu ya COVID-19.

\section{Washiriki:}

Shirika la Africa Humanitarian Action

Shirika la Alight

Shirika la Plan International

Baraza ya Idadi ya Watu

Shirika la Save the Children International

Kwa habari zaidi, wasiliana na:

Zahra Migrani: mirghani@unhcr.org

\section{Mfano wa Kisa kutoka Kenya:}

Kumudu Ushahidi wa baada ya Uchunguzi

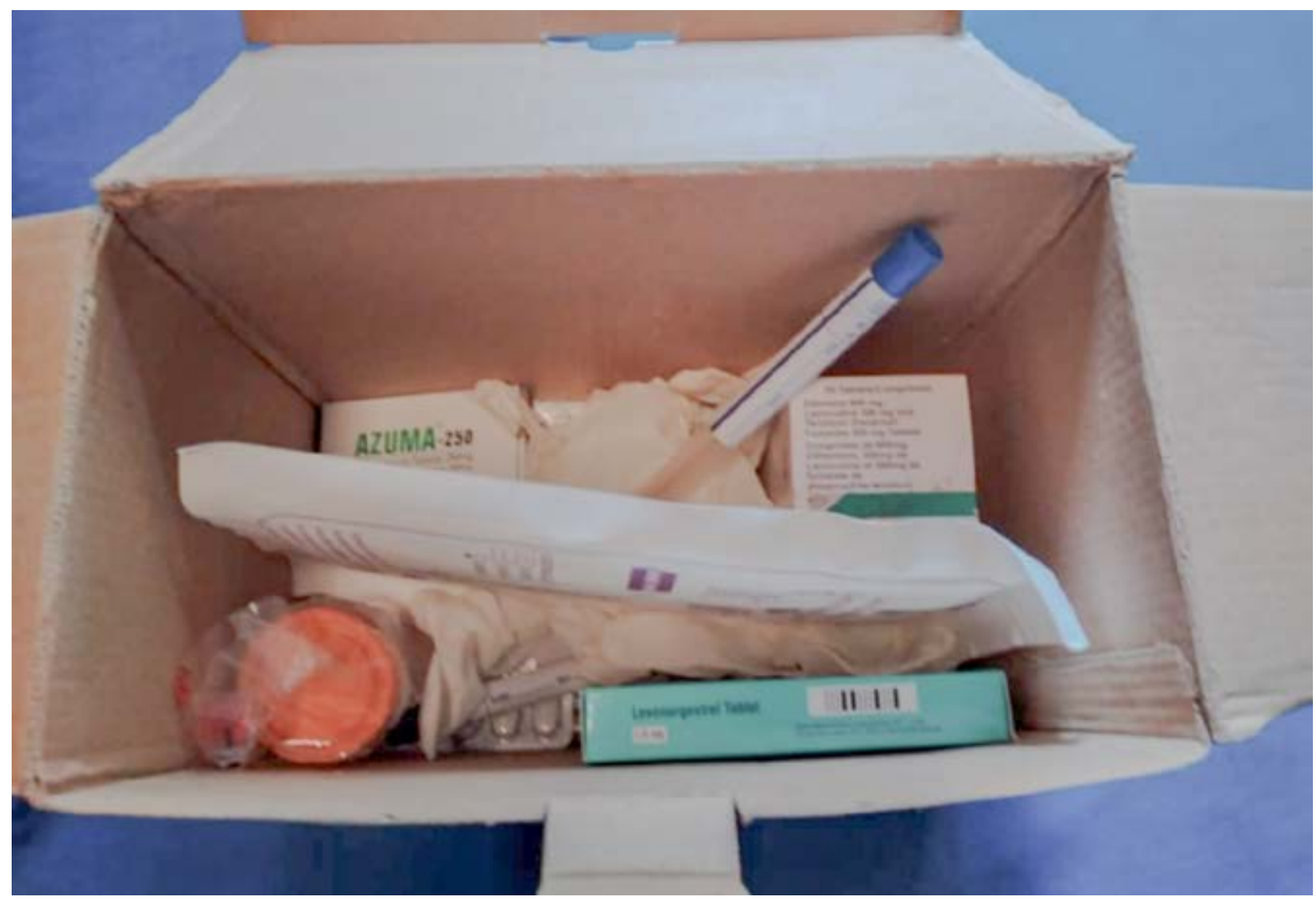




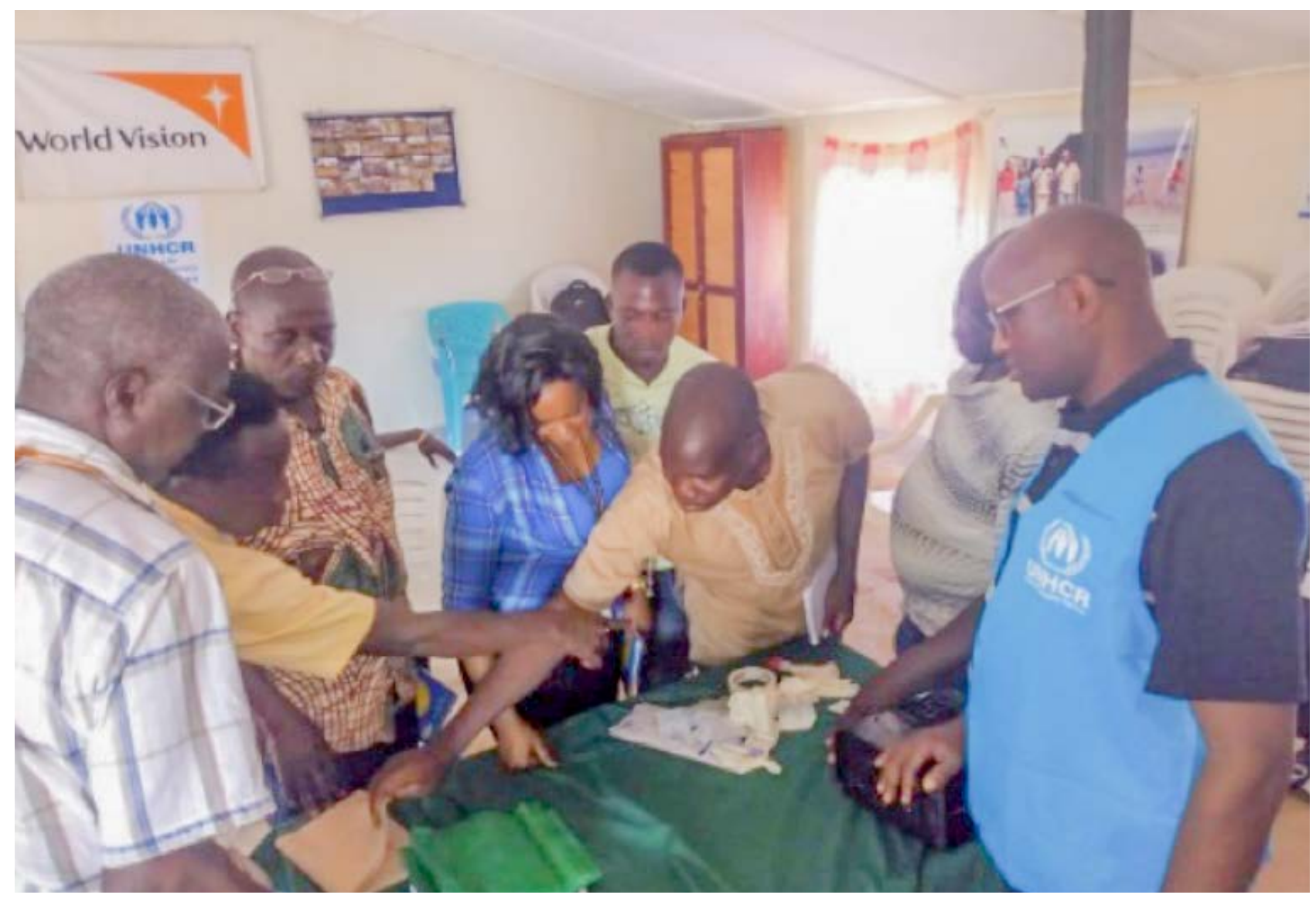

K

ama sehemu ya sekta tofauti za kukabiliana na SGBV, kupata huduma za kisheria unawezekana kwa wakimbizi walionusuriwa kulingana na mahitaji na uamuzi wao. Hata hivyo, mashtaka hutatizwa sana na ukosefu wa ushahidi wa uchunguzi wa ubakaji na visa vya dhulma ya kimwili. Kwa sababu hii, visa kadhaa hutupiliwa mbali na korti. Idara za polisi za Wilaya ya Kibondo na Kasalu zina Vitengo vya Ushahidi na huwa makini kusaidia kambi za wakimbizi. Upungufu hupatikana katika miktadha ya kambi hata hivyo, ikiwemo ukosefu wa vifaa vya utunzaji kutokana na Dhulma ya kimapenzi na kubakwa (PRC) katika vituo vyote vya kiafya, haja ya kusanifisha fomu ya PRC, hifadhi ya data isiyotosha ya fomu za uchunguzi wa kimatibabu wa polisi ('PF3') na ukusanyaji duni wa ushahidi wa vipengele vya uchunguzi wa kisheria.

Katika vituo vya polisi, kuna upungufu wa polisi wanaochunguza ushahidi wa kisheria katika maeneo yote ya kambi na ukosefu wa fedha za utathmini wa ushahidi wa kisheria kutoka eneo la tukio hadi duka la dawa la serikali. Kwa ujumla, ushirikiano miongoni mwa polisi, ulinzi, wahudumu wa afya ni dhaifu pamoja na ukosefu wa vifaa vya kukusanya na kuchambua vipengele vya uchunguzi wa kisheria katika vituo vya SGBV.

Katika kukabili hali hizi halisi, hatua ya kisasa inalenga kuboresha usimamizi wa ukusanyaji wa ushahidi wa uchunguzi wa kisheria kupitia kuimarisha huduma ya kiafya ya baada ya kubakwa na polisi kukusanya, kuandikisha na kutumia ushahidi wa uchunguzi. Hatua hii iliundwa na LVCT Health, nchini Kenya, na inajumuisha vigezo viwili muhimu: kuunda kifaa cha PRC kwa kutumia nyenzo zinazopatikana katika vituo vya afya na mafunzo ya sekta tofauti ya PRC, polisi, na wanasheria kuhusu jinsi ya kuhifadhi fomu zinazofaa kujazwa katika vituo vya afya kwa ushirikiano na polisi panapo visa vya ubakaji. Hifadhi hii ya data inahitaji kujazwa kwa umakini ili kuwezesha mashtaka ya ubakaji kortini. Fomu hizi (za huduma baada ya kubakwa, fomu za uchunguzi wa kimatibabu wa polisi) huwa na sehemu za wahudumu wa afya na polisi kujaza, ni muhimu wahudumu hawa wawili wajifahamishe kuhusu fomu hizi na jinsi ya kuzijaza ifaavyo, wakiwa na lengo la kuunganisha polisi/kituo cha afya (kulingana na alipopata huduma ya kwanza) na kufanya kazi pamoja kwa ushirikiano ili kuhakikisha fomu zimejazwa kwa ukamilifu ili kutumika katika uendeleshaji wa kesi.

Katika miktadha ya wakimbizi nchini Tanzania, hatua hii inatekelezwa ifuatavyo:

- Vifaa vya PRC vimeundwa katika nchi hiyo kwa ushirikiano wa karibu miongoni mwa vituo vyote vya afya.

- Fomu ya PRC ilisanifishwa kwa ajili ya matumizi katika kambi zote.

- Mafunzo kuhusu kukusanya ushahidi wa uchunguzi wa kisheria na jinsi ya kujaza fomu za PF3 kwa usahihi yalitekelezwa na wahudumu wa afya wa PRC.

- Polisi walipewa jukumu la kujumuisha uchunguzi wa kisheria katika hakiki ya risala ya maelewano kati ya UNHCR na polisi, mgao wa fedha za kutumika katika kutathmini ushahidi wa uchunguzi wa kisheria katika maeneo yote na kununua vifaa vya PRC ambavyo havikuwepo katika vituo vya afya. 
- Kitengo cha Ulinzi kilipewa jukumu la kuhakikisha kuwa washirika wahusika wanahudhuria mikutano ya uratibu ya kila mwezi ya SGBV SWG katika ngazi ya kambi; kuchangia katika kununua vifaa vinavyofaa na kuendesha vipindi vya kuhamasisha kuhusu jinsi ya kuchunguza ushahidi wa kisheria.

- Wahudumu sita wa kiafya wamefunzwa kuhusu usimamizi wa uchunguzi wa kisheria na wahudumu wa afya husika. Mada za mafunzo zilikuwa ukusanyaji wa ushahidi wa uchunguzi wa kisheria na mchakato wa kuunda vifaa vya PRC.

- Mazungumzo yenye mpangilio baina ya Wizara ya Mambo ya Ndani, Polisi na Duka la Dawa la Serikali katika maabara makuu ya uchambuzi ya Mwanza yalifanyika. Makubaliano yaliafikiwa katika misingi ya kuingilia kati, ikiwemo; mipangilio ya duka la dawa la serikali ya kuwafunza wahudumu wa kiafya katika usimamizi wa uchunguzi wa ushahidi wa kisheria na mipango ya serikali ya kuchunguza vituo vya afya kuhusu utayari wa usimamizi wa uchunguzi wa ushahidi wa kisheria (vifaa vya kukusanya, kuhifadhi na kadhalika)

Tangu kuanzilishwa kwa hatua hii, kumekuwa na maendeleo katika kujaza fomu za PF3 za polisi, ingawa vipindi vya mara kwa mara vya mafundisho vinapendekezwa kwa sababu ya polisi kuhamishwa kutoka kituo kimoja hadi kingine. Vifaa vya PRC vinapatikana katika vituo vya afya vinavyohusika na kambi na kuripoti kwa mara kwa mara na msaada kutoka UNHCR na vituo vya afya. Uratibu ulioboreshwa na Kitengo cha Ulinzi cha SGBV umeongeza mifumo ya kuelekeza manusura. Maendeleo yanaonekana pia katika wakati wa kuwajibikia manusura katika vituo tofauti vya kutoa huduma. Mikutano ya kila mwezi inayoandaliwa na UNHCR na washiriki muhimu hupewa jukumu la utathmini wa hatua (IRC, washiriki wa kiafya, maafisa wa serikali wa ulinzi, polisi) imewezesha kupatikana kwa suluhisho ya changamoto kadhaa.

\section{Washiriki:}

Shirika la International Rescue Committee

Afya ya LVCT

Timu za Kimatibabu za Kimataifa

Médecins Sans Frontières

Wizara ya Mambo ya Ndani

Idara ya Polisi

Mshiriki anayeshughulikia SGBV

Chama cha Msalaba Mwekundu cha Tanzania

\section{Kwa habari zaidi, wasiliana na:}

Benon Orach: orachb@unhcr.org

Rehema Katyega: katyega@unhcr.org

Dr. Yoel Mashimba: ymashimba@medicalteams.org

Miata Tubee Johnson: johnsonm@unhcr.org

Michael Gaitho: Michael.Gaitho@lvcthealth.org

\section{Desturi ya Kutia moyo kutoka nchi ya Uganda: ‘Muungano wa Kijiji wa Kukatiza Kuvumilia Dhulma}

M uungano wa Kijiji wa Kukatiza Kuvumilia Dhulma (ZTVA) ni njia moja ya jamii ya kuzuia Dhulma ya Kimapenzi na Kijinsia (SGBV) ambao uliundwa kwa ajili ya vijiji vidogo kule Afrika Kusini na Programu ya Uwezeshaji wa Waathirika wa Thohoyandou chini ya uongozi wa Fiona Nicholson, na ulitambulishwa kwa mara ya kwanza kwa makazi ya wakimbizi nchini Uganda. Makazi ya Rwamwanja ndio makazi ya kwanza kabisa ya msaada kwa wakimbizi kutokana na visa vingi vya SGBV vilivyoripotiwa.

\section{Mkakati huu unahusisha vipengele vifuatavyo:}

Mazungumzo na kuundwa kwa Ramani ya Jamii: ili kubaini miundo na mashirika ambayo yanaweza kulengwa kwa ajili ya kushirikishwa, ikijumuisha kutoa huduma za SGBV. Mazungumzo ya jamii hutumiwa kutoa taarifa kuhusu msaada na kukuza umiliki wa jamii.

Kuundwa kwa Baraza la Washikadau (SF): SF huteuliwa na wanachama wake hujumuisha sehemu zote za jamii (yaani, uongozi wa jamii, makanisa, elimu, biashara). SF huandaa mikutano ya kila mwezi ili kutathmini utekelezaji wa msaada, na kuhakikisha kuwa wanachama katika jamii wamehudhuria semina za mafunzo chini ya msaada huo.

Kutia sahihi Mkataba wa Makubaliano (MoA): Wanachama wa SF hutia sahihi kwa Mkataba wa Makubaliano ili kurasimisha 


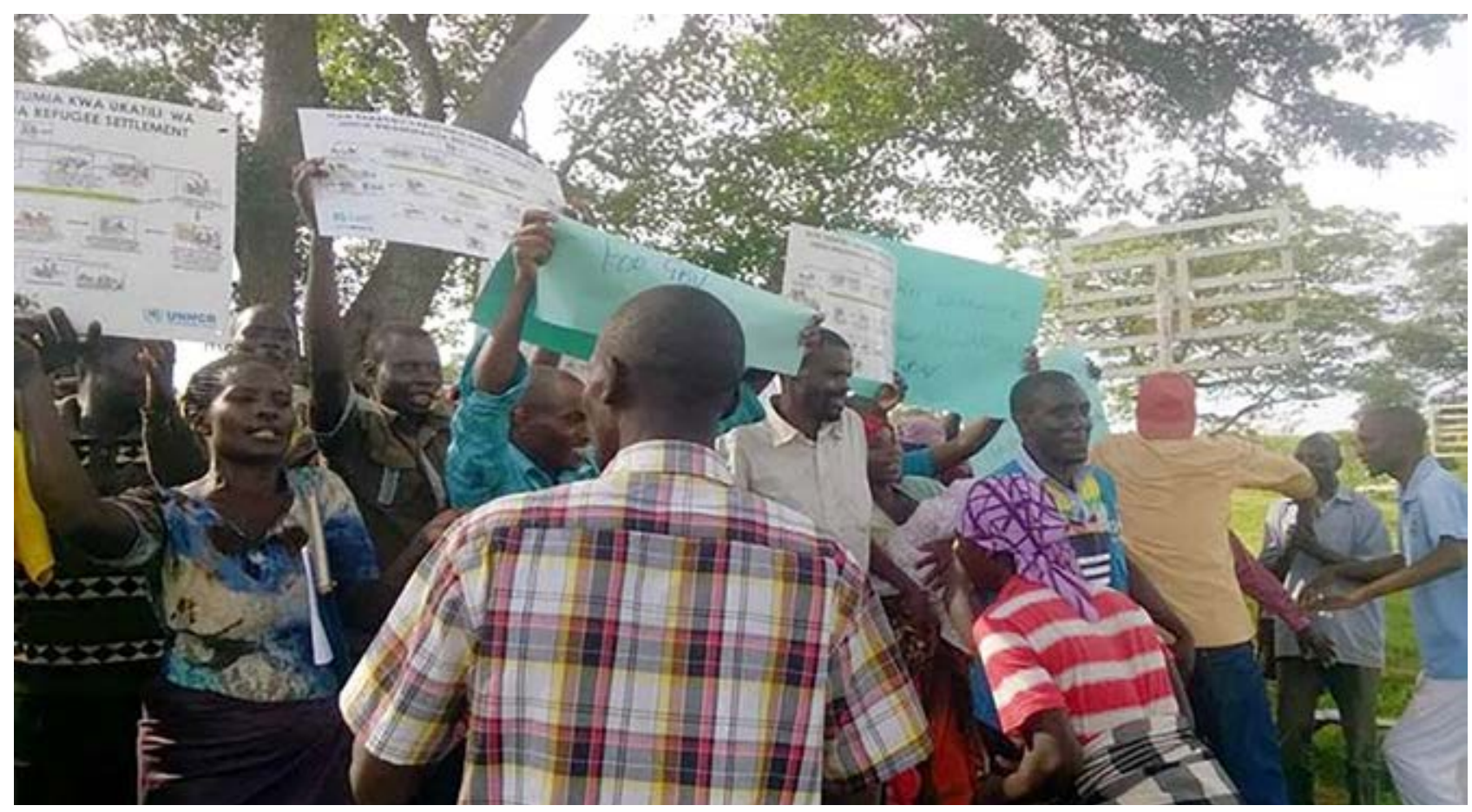

ushirikiano kati ya SF na mshirika anayetekeleza msaada. Mkataba huu wa Makubaliano huorodhesha vigezo ambavyo SF itazingatia ili kusaidia jamii kufikia na kuingizwa rasmi katika muungano wa vijiji ambavyo vinatajwa kama 'maeneo ambapo SGBV hairuhusiwi kabisa'.

Kutoa mafunzo: Makundi muhimu ya jamii huhamasishwa kuhusu masuala ya SGBV kupitia vikao vya mafunzo. Vijitabu vinavyoeleza kwa ujumla mafunzo yaliyotolewa hupeanwa, na wanafunzi wanahimizwa kufunza mtu mwingine mmoja au wawili katika jamii baada ya kupata mafunzo wao wenyewe.

Sherehe ya Kuapisha: Mara baada ya matarajio yaliyoainishwa katika Mkataba wa Makubaliano kutekelezwa na kijiji husika, kijiji hicho hupokea udhamini ili kuandaa sherehe ya umma, na hapa ndipo wanaume katika jamii hiyo wanaalikwa kuapa hadharani kuwa watahusika katika kutokomeza SGBV vijijini mwao, na zawadi hupeanwa kwa wanachama kadhaa wa jamii ambao wameshika msimamo dhidi ya SGBV, au kutoa shuhuda zao.

Kutambulishwa kwa Muungano: Sherehe ya Kuapisha humalizika kwa kuzinduliwa kwa bango la jamii, linaloonyesha jina la kijiji hicho na linalotangaza hali yao ya 'kutostahimili kabisa' na kujumuishwa rasmi katika 'muungano' wa vijiji 'visivyostahimili kabisa.' Nafasi kidogo ilitolewa pia kwa ajili ya vijiji kutumia kukuza ujamii baada ya sherehe.

Matokeo ya utathmini wa utekelezaji wa msaada huu katika makazi ya wakimbizi nchini Uganda yameonyesha kupunguka kwa kiasi kikubwa kwa matukio ya: Dhulma ya kudhuru mwili unaotekelezwa kati ya wapenzi (IPV) (wanaume na wanawake), Dhulma ya Kimapenzi wa IPV (kwa wanaume), Dhulma ya kudhuru mwili miongoni mwa watu ambao sio wapenzi (kwa wanaume na wanawake), na Dhulma ya Kimapenzi miongoni mwa watu wasio wapenzi (kwa wanawake).

Hadi sasa, msaada wa ZTVA umetekelezwa katika takriban vijiji 15 vya wakimbizi nchini Uganda. Matayarisho ya awali (yanajumuisha kutoa mafunzo kwa washirika wanaohusika na utekelezaji, uhamasishaji wa viongozi wa jamii, na/au uundaji wa Mabaraza ya Washikadau, na kadhalika.) vilevile umefanywa ili kutambulisha msaada husika katika makazi ya wakimbizi kule Djibouti, Ethiopia, na Zambia

\section{Washiriki:}

Shirika la Lutheran World Federation

Ofisi ya Waziri Mkuu

Kwa habari zaidi, wasiliana na:

Mildred Ouma: ouma@unhcr.org

Monicah Rwotmon: monicah.rwotmon@lutheranworld.org

Fiona Nicholson: nicholsonfiona27@gmail.com 


\section{Mfano wa Kisa kutoka nchi ya Sudan Kusini: Mbinu za Jamii katika Kujilinda (Kinga ya SGBV, Kupunguza Hatari, Ulinzi wa Watoto, na Ushiriki wa Vijana)}

W

akimbizi katika Jimbo la Upper Nile hukaa katika kambi nne katika Kaunti ya Maban, na hutegemea sana mashirika ya kibinadamu kutoa huduma za kuokoa maisha na huduma za kimsingi. Huku maendeleo makubwa yakiwa tayari yamefanywa, wasiwasi kuhusiana na visa vya SGBV, kama vile ndoa za kulazimishwa, ubakaji, ukeketaji, na kunyimwa fursa kwa wanawake na wasichana zinaendelea kuripotiwa. Isitoshe, uwepo wa silaha umeathiri tabia ya raia wanaohitaji hifadhi na ya kibinadamu ya hifadhi na kuchangia kutokea kwa visa vingine vya SGBV.

Misaada ya aina mbalimbali hivyo basi ilibuniwa ikiwa na malengo ya: kuhakikisha usalama wa manusura wa SGBV katika kambi za Wakimbizi za Maban (pamoja na, lakini sio tu, kutoa makazi salama ya jamii); kuimarisha utangamano, kubuni au kuboresha njia za mawasiliano, kukuza ujasiri katika utetezi na upashanaji habari; na kujenga uwezo wa miundo ya jamii katika kutambua kwa wakati nzuri, kuwasilisha, na kukabiliana na mahitaji yanayotokea katika jamii.

Ili kushirikisha kwa ufanisi miundo ya jamii katika kufikia malengo haya, UNHCR, pamoja na SGBV na Ulinzi wa Watoto na Washirika wa Vijana, imeendelea kuimarisha uwezo wa watetezi wa SGBV, Wanaharakati wa SASA!*, viongozi wa wakimbizi, wawakilishi wa wanawake na vijana, Wanaharakati wa Jamii, na wawakilishi wa Makao Salama ya Jamii ili kuongeza mchango wao kwa kuzuia, kupunguza na kukabiliana na masuala ya SGBV kwa kushirikiana na UNHCR na washirika. Mikakati Maalum inajumuisha:

- Kuanzishwa kwa Makao Salama ya Jamii - mpango unaoendeshwa na jamii - kuhakikisha kuwa manusura hawako katika hali ya hatari.

- Shughuli zinazoendeshwa katika kituo cha wanawake (vipindi vya kusikiliza vya wanawake na wasichana), ambazo ni jukwaa salama la kujadili masuala yanayowakabili, na vilevile jukwaa la kujadili mapendekezo ya jinsi ya kukabiliana na masuala hayo.

- Kushirikisha jamii nzima katika kukabiliana na kukinga SGBV pamoja na kujumuisha vijana na watoto katika mahali wanakoishi, wanakocheza, wanakofanya kazi na wanakopata mafunzo.

- Programu madhubuti ya SGBV inayojumuisha mbinu ya SASA!, 'jamii nzima', mbinu ya kijamii ambapo wanaharakati wa SASA!, Wachunguzi wa Ulinzi, Mawakili wa SGBV, Viongozi wa Jamii, Wawakilishi wa Chama, na viongozi wa dini wote wanahusika katika kuhamasisha na kusambaza habari kuhusu SGBV kijijini.

- Ushiriki wa makusudi wa wavulana na wanaume katika mpango wa SGBV kama vichocheo vya mabadiliko mazuri ya tabia.

- Udhibiti wa Visa kwa kuzingatia vigezo muhimu vya washirika ili kuhakikisha njia mbali mbali za kukabiliana na visa hivyo.

- Kutoa hema na vitu visivyo vya chakula.

\section{Washiriki:}

Tume ya Masuala ya Wakimbizi

Mamlaka katika Kaunti

Baraza la Wakimbizi nchini Denmark

Wizara ya Jinsia, Watoto na Ustawi wa Jamii

Shirika la Relief International

Shirika la Save the Children International

Kwa habari zaidi, wasiliana na:

SO Bunj AoR 
